\title{
Spindynamik in Tunnelelementen mit senkrechter magnetischer Anisotropie
}

\author{
Dissertation \\ zur Erlangung des mathematisch-naturwissenschaftlichen \\ Doktorgrades \\ "Doctor rerum naturalium" \\ der Georg-August-Universität Göttingen \\ im Promotionsprogramm ProPhys \\ der Georg-August University School of Science (GAUSS)
}

\author{
vorgelegt von \\ Vladyslav Zbarsky \\ aus Dnjepropjetrowsk (Ukraine)
}

Göttingen, 2014 


\section{Betreuungsausschuss:}

Prof. Dr. Markus Münzenberg, I. Physikalisches Institut

Prof. Dr. Christian Jooß, Institut für Materialphysik

\section{Mitglieder der Prüfungskommission:}

Referent/in

Prof. Dr. Markus Münzenberg, Physikalisches Institut, Universität Greifswald

Korreferent/in

Prof. Dr. Christian Jooß, Institut für Materialphysik

Weitere Mitglieder der Prüfungskommission:

Prof. Dr. Michael Seibt, IV. Physikalisches Institut

Prof. Dr. Vasily Moshnyaga, I. Physikalisches Institut

Prof. Dr. Wolfram Kollatschny, Institut für Astrophysik

Prof. Dr. Hans-Ulrich Krebs, Institut für Materialphysik

Tag der mündlichen Prüfung: 22.01.2015 
„Jedes Naturgesetz, das sich dem Beobachter offenbart, lässt auf ein höheres, noch unerkanntes schließen."

Alexander von Humboldt 



\section{Inhaltsverzeichnis}

$\begin{array}{lr}\text { Abstract } & 1\end{array}$

1. Einführung 3

1.1. Motivation ....................... 3

1.2. Spintronik . . . . . . . . . . . . . . . . 4

1.3. Gliederung und Ziele dieser Arbeit . . . . . . . . . . . . . . 5

2. Theorie 7

2.1. Ferromagnetismus . . . . . . . . . . . . . . . . 7

2.2. Magnetische Tunnelelemente . . . . . . . . . . . . . . . 9

2.2.1. TMR-Effekt . . . . . . . . . . . . . . . 99 9

2.2.2. Brinkman-Modell . . . . . . . . . . . . . . . . . . . . . 10

2.2.3. Kohärentes Tunneln . . . . . . . . . . . . . . . . . . 12

2.3. Magnetische Anisotropie . . . . . . . . . . . . . . . . . . . . . 14

2.4. Stoner-Wohlfahrth-Modell . . . . . . . . . . . . . . . . . 18

2.5. Spin-transfer torque . . . . . . . . . . . . . . . . . . . 20

2.5.1. Mechanismus des Stromschaltens . . . . . . . . . . 20

2.5.2. STT an magnetischen Tunnelelementen mit PMA . . . . . . . 21

2.6. Dynamik an den MTJs . . . . . . . . . . . . . . . . 22

3. Experimentelle Methoden $\quad 27$

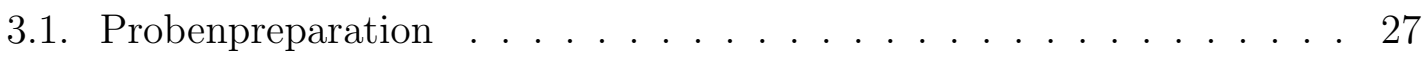

3.1.1. Substratreinigung . . . . . . . . . . . . . . 27

3.1.2. Schichtenwachstum . . . . . . . . . . . . . . 27

3.1.3. Auslagerung der Proben . . . . . . . . . . . . . . . . 30

3.2. Strukturierung . . . . . . . . . . . . . . . . . 31

3.2.1. Lithographie . . . . . . . . . . . . . . . 31

3.2.2. Sputterätzen . . . . . . . . . . . . . . . 32

3.2.3. Strukturierung mehrerer Kontakte . . . . . . . . . . . . . . 32

3.3. Charakterisierung . . . . . . . . . . . . . . . . 34

3.3.1. TMR und STT . . . . . . . . . . . . . . . . 34

3.3.2. Messung des magnetooptischen Kerr-Effekts . . . . . . . . . . 35

3.3.3. Zeitaufgelöster magnetooptischer Kerr-Effekt . . . . . . . . . . 36 
4. Experimentelle Ergebnisse $\quad 39$

4.1. Magnetische Anisotropie der CoFeB/MgO-basierten Tunnelelemente. 40

4.1.1. Vorwort . . . . . . . . . . . . . . . . . 40

4.1.2. Abhängigkeit der Magnetischen Anisotropie von der CoFeB-

Schichtdicke. . . . . . . . . . . . . . 40

4.1.3. Einfluss der Diffusionsvorgänge auf die magnetischen Eigenschaften der magnetischen Tunnelelemente . . . . . . . . . . . 46

4.2. Spin-transfer torque . . . . . . . . . . . . . . . . . . . . . 49

4.2.1. Qualität der in- und out-of-plane Proben mit TMR als Qualitätsmerkmal. . . . . . . . . . . . . . . . 49

4.2.2. Spin-transfer torque an Tunnelelementen mit PMA . . . . . . 54

4.2.3. Thermische Stabilität . . . . . . . . . . . . . . 58

4.3. Dynamik an MTJs mit PMA . . . . . . . . . . . . . . 59

4.3.1. Spinstrompulse . . . . . . . . . . . . . . 6 60

4.3.2. Magnetisches Schalten der MTJs . . . . . . . . . . . . . 61

4.3.3. Magnetisches Verhalten der MTJs auf ultrakurzen Zeitskalen . 63

5. Zusammenfassung und Ausblick $\quad \mathbf{7 3}$

5.1. Magnetische Anisotropie der CoFeB-Schicht . . . . . . . . . . . . 73

5.2. Spin-transfer torque . . . . . . . . . . . . . . . . . 74

5.3. Dynamik auf ultrakurzen Zeitskalen . . . . . . . . . . . . . . 76

$\begin{array}{ll}\text { A. Weitere Messungen und Auswertungsschritte } & \mathbf{7 9}\end{array}$

A.1. MOKE-Messungen der Probe B . . . . . . . . . . . . . . . . . . . . . 79

A.2. Normierung der Dynamikmesskurven . . . . . . . . . . . . . 80

A.3. Demagnetisierungsfit für Positionen C und D . . . . . . . . . . . . . 80

$\begin{array}{lc}\text { Literaturverzeichnis } & 83\end{array}$

$\begin{array}{lr}\text { Nomenclature } & 89\end{array}$ 


\section{Abstract}

The modern world can not be imagined without computer technology anymore. Due to modern devices such as computer or smart phone it is possible to have an access to a huge amount of information contained in the world wide web. Nowadays it is practicable to exchange information and work on the same projects from nearly every part of the world. However, the need to exchange and save such huge amount of data leads to the increased requirements of modern hardware. Hard drive discs and random access memory need more and more memory cells, which can be rewritten and be read faster and faster, because slower memory cells can not save the still increasing amount of data without slowing down the data processing. Another crucial parameters which also need to be optimized for the competitive memory units are energy efficiency and of course the cost factor.

During the last thirty years the memory space per square centimeter on the memory unit was increased exponential. The reason for such strong improvement is the continuous decreasing of the unit cells down to nanometer scale [1], which in turn is the result of many years research of new materials and their applications. During this time a lot of new methods for memory cell fabrication, patterning and read/write processes were developed and optimized [2]. On the field of energy efficiency there was also a huge progress, so for example the development of the modern spin-transfer torque elements (STT-elements) leads to decreasing by the factor $\sim 1.5$ (compared with static CMOS) of the energy needed to switch the memory cell from "0" to " 1 " (or vice versa) [3, 4]. The rewriting and reading speed of the memory cells measured on the STT-junctions is in the area of few nanoseconds (newest cells even $<1 \mathrm{ns)}$, which makes STT-devices comparable with conventional memory cells $[1,3,4]$. All this advantages make the magnetic tunnel junctions (MTJs) very attractive for applications. So the investigation and optimization of the $\mathrm{CoFeB} / \mathrm{MgO}$-junctions is of current interest and is also the central issue of this work. Especially the interest lies on the junctions with perpendicular magnetic anisotropy and their advantages over the MTJs with in-plane magnetic anisotropy.

The first chapter of this work is concerned with the theoretical background of the following experimental work. The fabrication methods of the full layer stacks, their patterning methods and characterization setups are presented in the chapter "Experimentelle Methoden". The magnetic anisotropy behavior of the samples fabricated in our group will be measured and discussed in the chapter "Auswertung". First measurement of the Spin-Transfer-Torque and the comparison of the switching current behavior with the literature is also presented in the chapter "Auswertung". 
The discussion contains also results of the measurements on the ultra short time scales, where the minority electrons spin channeling was observed. 


\section{Einführung}

\subsection{Motivation}

Der Tunnelmagnetowiderstand (engl. tunnel magneto resistance, kurz TMR) beschreibt den magnetfeldabhängigen Widerstand eines Tunnelelements, welcher aus zwei dünnen ferromagnetischen Schichten getrennt von einer dünnen isolierenden Barriere besteht. Zum ersten Mal wurde dieser Effekt von M. Julliere im Jahr 1975 in Fe/Ge/Co - Tunnelelementen bei 4,2 K entdeckt. Der damals gemessene TMRWert lag bei $14 \%$ [5]. Für Anwendungen eignete sich diese Entdeckung noch nicht, da er nur bei tiefen Temperaturen gemessen werden konnte. Erst nach der Entdeckung des GMR-Effekts (engl. Giant magnetoresistance) von A. Fert [6] (1988) und P. Grünberg [7] (1989) kehrte das Interesse an der Erforschung des Tunnelmagnetowiderstands zurück: der GMR-Effekt wurde relativ schnell in Anwendungen wie z. B. Festplattenleseköpfe integriert. Die beiden Forscher A. Fert und P. Grünberg wurden für das Entdecken des GMR-Effekts 2007 mit dem Nobelpreis ausgezeichnet.

Einen weiteren Durchbruch auf diesem Feld brachten die Messungen von J. S. Moodera [8] und T. Miyazaki [9], die einen TMR-Wert von $18 \%$ bei Raumtemperatur gemessen haben. Diese Messungen wurden an Tunnelelementen mit einer amorphen Aluminiumoxid-Barriere durchgeführt. Weitere Optimierung der Tunnelelemente hat diesen Wert sogar auf etwa $70 \%$ bei Raumtemperatur erhöht.

Die Berechnungen von W. Butler et al. [10], J. Mathon und A. Umerski et al. [11] zeigten im Jahr 2001, dass der TMR-Wert sogar bei über $1000 \%$ liegen kann, wenn statt einer amorphen Aluminiumoxid-Barriere eine kristalline $\mathrm{MgO}$-Barriere verwendet wird. Drei Jahre nach den theoretischen Berechnungen von W. Butler et al. und J. Mathon et al. wurde im Jahr 2004 an einem Fe/MgO/Fe-Tunnelkontakt von S. Yuasa et al. ein TMR-Wert von $88 \%$ gemessen [12]. Eine weitere Erhöhung des TMR-Wertes versprach die Verwendung einer Kobalt-Eisen-Legierung als ferromagnetische Schicht, denn die amorph wachsende CoFe-Schicht kristallisiert erst während der Auslagerung bei hohen Temperaturen und passt ihre Kristalleigenschaften an die kristalline MgO-Barriere an. Der bis 2014 höchste experimentell erreichte TMR-Wert wurde an einem Element mit CoFeB/ MgO/ CoFeB-Zusammensetzung von S. Ikeda et al. [13] gemessen und beträgt bei Raumtemperatur $604 \%$. 


\subsection{Spintronik}

Unsere moderne Welt ist ohne Computer-Technologie nicht mehr vorstellbar. Mit Hilfe der modernen Technologien hat man Zugang zu enormen Mengen an Information, denn heutzutage ist es möglich beinah von jedem Ort Information auszutauschen, was die Arbeit an gemeinsamen Projekten aus der ganzen Welt ermöglicht und erleichtert. Jedoch gilt: Je mehr Daten schnell bearbeitet und ausgetauscht werden sollen, desto höher sind die Anforderungen an die Hardware der modernen Rechner. So müssen moderne Speicherzellen viel Speicherplatz auf kleinem Raum zur Verfügung stellen und es soll möglichst schnell auf die Speicherzellen zugegriffen werden. Außerdem dürfen zusätzlich die Energieeffizienz und der Kostenfaktor nicht vernachlässigt werden. Alle diese Faktoren sind entscheidend im Wettbewerb und müssen bei der Produktherstellung berücksichtigt werden.

In den letzten dreißig Jahren vergrößerte sich der Speicherplatz der modernen Festplatten um mehrere Größenordnungen. Dies konnte durch die Verkleinerung der Bit-Größe erreicht werden [1], was wiederum ein Ergebnis jahrelanger Erforschung neuer Materialien und deren Anwendungen ist. Der Schritt zu Speicherzellen von etwa $100 \mathrm{~nm}$ Größe erlaubte eine deutliche Erhöhung der Bit-Dichte auf dem Speichermedium. Es wurden mehrere Methoden zur Herstellung, Strukturierung und Kontaktierung solcher Bit-Felder entwickelt und optimiert. Auch auf dem Feld der Energieeffizienz wurde einiges getan, zum Beispiel die Entwicklung der SpinTransfer-Torque (kurz STT) Schaltelementen verspricht für neue Speicherelemente um einige Größenordnungen sparsameren Energieverbrauch als ihre Vorgänger [3]. Die Forschungsergebnisse der letzten Jahre [14] zeigen, dass sogar die bereits sparsamen STT-Schaltelemente weiterhin effizienter werden können. Dafür müssen die STT-Tunnelelemente mit einer senkrechten magnetischen Anisotropie der ferromagnetischen Schichten verwendet werden. Die senkrechte magnetische Anisotropie verspricht eine weitere Verringerung der Schaltströme [14] und somit eine Steigung der Energieeffizienz. Aus diesem Grund behandelt diese Arbeit insbesondere die magnetischen Tunnelelemente mit einer senkrechten magnetischen Anisotropie und die Parameter von denen die Anisotropie abhängig ist.

Im Bereich Schreib- und Lesegeschwindigkeiten gab es durch STT in den letzten Jahren auch viele Fortschritte. Zum Beispiel die Zugriffsgeschwindigkeit der auf dem STT - basierenden Elemente beträgt bereits nur wenige Nanosekunden [3, 1].

Diese Vorteile können in mehreren technischen Anwendungen genutzt werden. Ein prominentes Beispiel für eine interessante moderne Anwendung aus dem Gebiet der Spintronik (eine Zusammensetzung aus Spin und Elektronik) ist MRAM (engl. Magnetoresistive Random Access Memory). Bei dem MRAM handelt es sich um einen nichtflüchtigen Arbeitsspeicher, der nach dem Abspeichern der Daten ohne Stromzufuhr erhalten kann.

Durch viele Anwendungsmöglichkeiten wird das Gebiet der Spintronik zu einem sehr aktuellen und viel versprechenden Forschungsfeld. Diese Arbeit deckt einen Teil dieses Forschungsgebiets ab und behandelt einige aktuelle Herausforderungen. 


\subsection{Gliederung und Ziele dieser Arbeit}

Diese Arbeit beschäftigt sich mit den magnetischen Eigenschaften der aus KobaltEisen-Bor-Legierung und Magnesiumoxid hergestellten Tunnelelementen. Der Schwerpunkt dieser Arbeit liegt auf der Untersuchung der Tunnelelemente mit einer zur Probenoberfläche senkrechten Anisotropie. Eine kontrollierte Nutzung der magnetischen Anisotropie und der damit verbundenen Effekte verspricht eine Effizienzsteigerung der auf Tunnelelementen basierten Anwendungen. Aus diesem Grund ist der Übergang der magnetischen Anisotropie von der senkrechten zur parallelen Ausrichtung bezüglich der Probenoberfläche von besonderem Interesse. Die magnetischen und elektrischen Eigenschaften der Tunnelelemente sollen in dieser Arbeit nach der Zusammenstellung eines Schichtenstapels mit senkrechter magnetischen Anisotropie untersucht und diskutiert werden.

In Kapitel 2 werden theoretische Grundlagen behandelt. Es wird ein Überblick über die relevanten Effekte gegeben. Insbesondere wird auf die Eigenschaften der Tunnelelemente eingegangen, die für diese Arbeit von zentraler Bedeutung sind.

In dem Kapitel 3 werden die experimentellen Methoden erläutert. Dafür werden kurz der verwendete Probenherstellungsprozess, die Strukturierungs- und Charakterisierungsmethoden erläutert.

Die Messdaten, deren Analyse und Vergleich mit aktuellen Forschungsergebnissen werden in dem Kapitel 4 präsentiert. Die Zusammenfassung und eine weitere kurze Diskussion der Ergebnisse wird in dem letzten Kapitel stattfinden. Außerdem werden in diesem Kapitel Vorschläge für zukünftige Arbeiten gemacht.

$\mathrm{Zu}$ den Hauptzielen dieser Arbeit gehören:

- Herstellung der $\mathrm{CoFeB} / \mathrm{MgO}$-basierten Schichtsysteme mit unterschiedlicher magnetischer Anisotropie.

- Vermessung und Untersuchung der magnetischen Anisotropie der $\mathrm{CoFeB} / \mathrm{MgO}$ basierten Schichtenstapeln in Abhängigkeit von der CoFeB-Schichtdicke und Pufferschicht.

- Erste Untersuchungen der in dieser Arbeitsgruppe hergestellten Spin-TransferTorque-Tunnelelemente mit senkrechter magnetischer Anisotropie.

- Messungen und Analyse des Spintransports auf ultrakurzen Zeitskalen an den Proben mit senkrechter Anisotropie. 



\section{Theorie}

In diesem Kapitel werden die relevanten Effekte und Relationen kurz beschrieben und erklärt. Das Ziel dieses Kapitels ist einen Überblick über den aktuellen Stand und Herausforderungen auf jeweiligen Gebieten zu geben. Einige Effekte und Relationen wurden bereits unter anderen Blickwinkeln von meinen Vorgängern M. Walter [15], G. Eilers [16] und J. Walowski [17] diskutiert. An den Stellen, bei denen die theoretischen Methoden bereits ausführlich von den Vorgängern diskutiert worden sind, wurde auf besonders detaillierte Beschreibung der Effekte verzichtet. Es werden jedoch zu allen relevanten Themen zumindest theoretische Grundlagen als Überblick gezeigt.

\subsection{Ferromagnetismus}

In dieser Arbeit spielt Ferromagnetismus eine entscheidende Rolle, da insbesondere einige Eigenschaften solcher Ferromagneten wie Eisen, Kobalt und deren Legierungen werden unter dem Blickwinkel der technologischen Relevanz und Interesse untersucht. In diesem Kapitel werden einige relevante Eigenschaften dieser Materialien präsentiert.

Bringt man ein ferromagnetisches Material in ein Magnetfeld, so zeigt ein Ferromagnet nach dem Abschalten des externen Feldes eine von Null unterschiedliche innere Magnetisierung. Diesen Effekt haben die Ferromagneten der Bildung magnetischer Domänen mit gleicher Magnetisierungsrichtung innerhalb der Domäne zu verdanken. Betrachtet man also einen Ferromagneten ohne magnetische Vorgeschichte, so gleichen sich die magnetischen Domänen (auch Weiss-Bezirke genannt) im Mittel aus. Wird jedoch ein äußeres Magnetfeld angelegt, so ändern immer mehr Domänen ihr inneres Magnetfeld parallel zum äußeren Feld. Beim Ausschalten des externen Magnetfeldes bleibt die Ausrichtung der Weiss-Bezirke enthalten und das Material zeigt eine Restmagnetisierung (genannt Remanenz). Um das innere Magnetfeld des Ferromagneten wieder auf Null zu bringen, muss ein äußeres Feld entgegen der Remanenzrichtung wirken. Die in diesem Fall benötigte Magnetfeldstärke wird als Koerzitivfeldstärke bezeichnet [18]. 


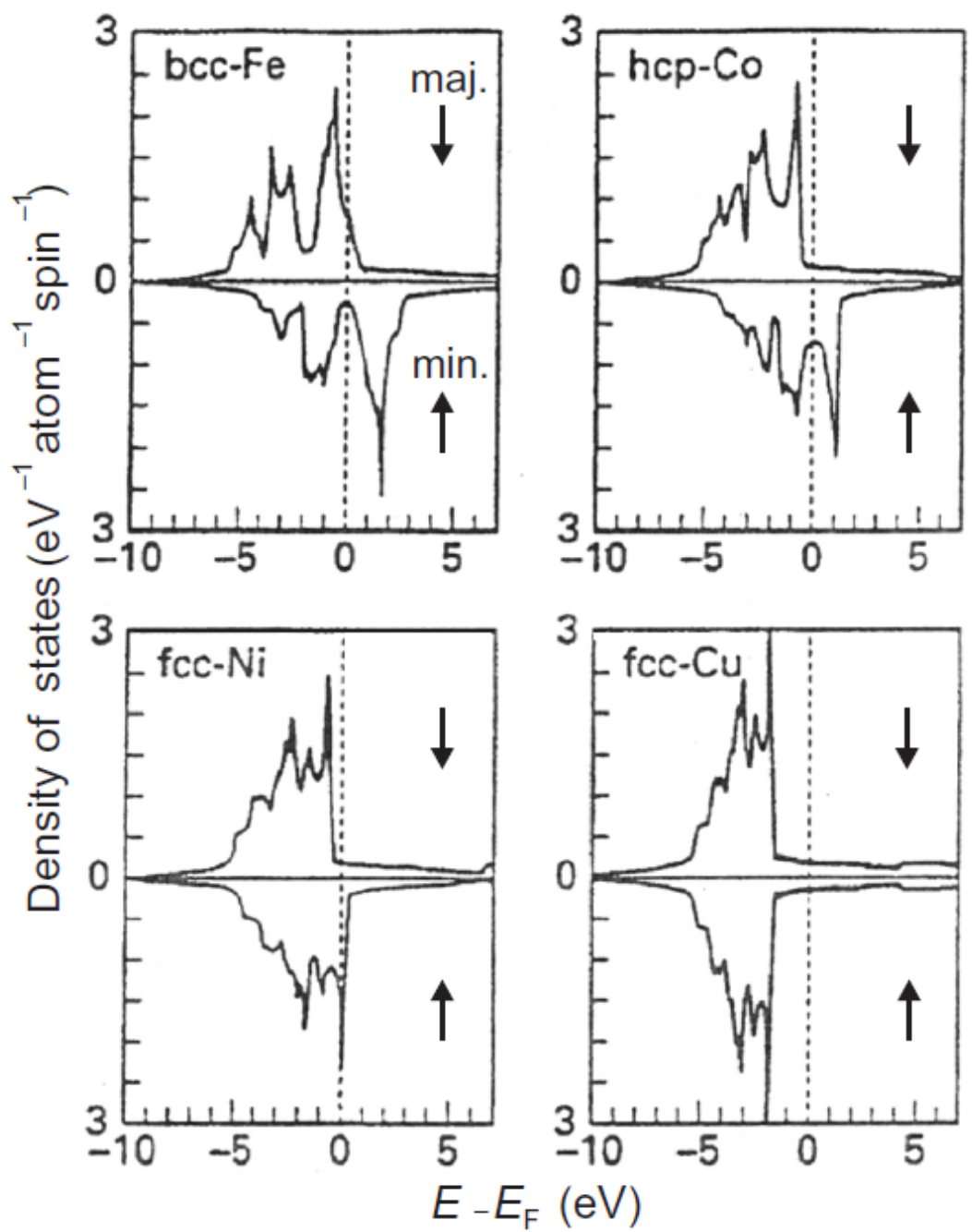

Abbildung 2.1.: Stoner-Modell. Zustandsdichten der 3d-Metalle für Majoritätsund Minoritätselektronen: $\mathrm{Fe}, \mathrm{Co}, \mathrm{Ni}$ und $\mathrm{Cu}$ [19]. Kupfer ist kein Ferromagnet und wird hier nur als Vergleich gezeigt.

In dieser Arbeit werden als ferromagnetische Schicht eine Legierung aus den 3dÜbergangsmetallen Co und Fe verwendet. Die Besonderheit der Metalle aus der IV. Periode des Periodensystems mit der teilweise besetzten 3d-Schale liegt darin, dass die 3d-Orbitalen durch die Austauschwechselwirkung eine spinabhängige Zustandsdichtenaufspaltung aufweisen (siehe Abbildung 2.1). Diese Eigenschaft führt dazu, dass bei der Besetzung der Zustände niedrigerer Energie (also nur bis zur Fermi-Energie) eine Spinausrichtung stärker repräsentiert wird. Die Elektronen mit einer höheren Zustandsdichte für eine bestimmte Spinausrichtung werden als Majoritätselektronen bezeichnet. Die Elektronen mit der schwächer repräsentierten Spinausrichtung werden als Minoritätselektronen bezeichnet. Dies führt zu einem pro Atom nicht verschwindenden magnetischen Beitrag, der durch den Überschuss der Majoritätsspins entsteht, der wiederum makroskopisch durch die Domänenbildung 
ausgeglichen werden kann.

\subsection{Magnetische Tunnelelemente}

\subsubsection{TMR-Effekt}
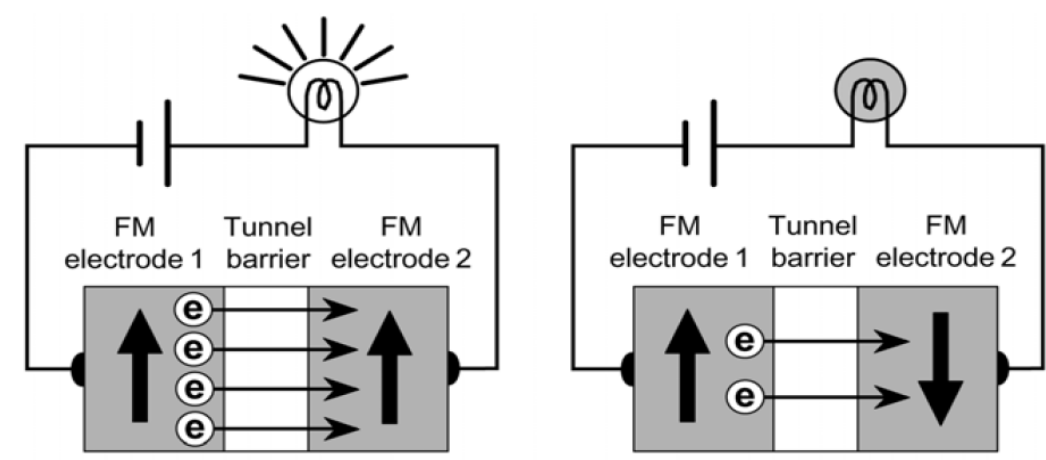

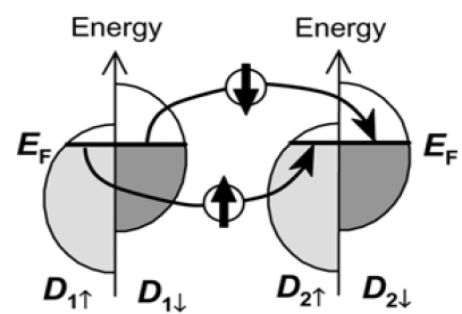

(a)

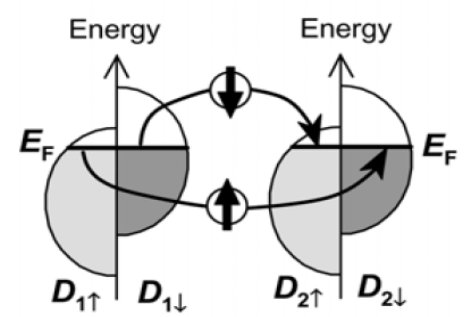

(b)

Abbildung 2.2.: Veranschaulichung des TMR Effektes: (links) der Zustand der parallelen magnetischen Ausrichtung, (rechts) der antiparallele Zustand mit einer Widerstandserhöhung [20].

Ein magnetisches Tunnelelement (engl. magnetic tunnel junction, kurz MTJ) wird aus zwei dünnen ferromagnetischen Elektroden getrennt durch eine dünne isolierende Barriere hergestellt. Die ferromagnetische Schichten können in zwei Zustände gebracht werden: parallel (auch P-Zustand genannt) und antiparallel (auch APZustand genannt) zu einander (siehe Abbildung 2.2). Der Widerstand der MTJs ist abhängig von dem magnetischen Zustand der ferromagnetischen Schichten und als Tunnelmagnetowiderstand (engl. tunnel magnetoresistance, kurz TMR) bezeichnet. Der Ursprung der Widerstandsabhängigkeit von der Magnetisierung der Ferromagneten liegt an der bereits in Abschnitt 2.1 erwähnten spinabhängigen Aufspaltung der Zustandsdichten ferromagnetischer Materialien. Diese führt zur unterschiedlichen Besetzung der Energiebänder für beide Elektroden im AP-Zustand (siehe Abbildung 2.2): es können nur Majoritätselektronen von einer Elektrode in die Minoritätszustände der Elektronen der anderen Elektrode tunneln. Dies reduziert stark die Anzahl der tunnelnden Elektronen und kann als Absenkung der Stromstärke 
(bzw. Erhöhung des Widerstandes) gemessen werden. Der TMR-Wert ist folgendermaßen definiert [8, 21]:

$$
T M R=\frac{R_{A P}-R_{P}}{R_{P}},
$$

wobei $R_{A P}$ der Widerstand im antiparallelen und $R_{P}$ im parallelen magnetischen Zustand sind. Da der TMR-Wert von der Spinpolarisation der beiden Elektronen abhängig ist, kann eine weitere Formel zur Berechnung des TMR-Wertes verwendet werden [5]:

$$
T M R=\frac{2 P_{1} P_{2}}{1-P_{1} P_{2}}
$$

mit $P_{1}$ und $P_{2}$ - Polarisation zum Tunnelprozess beitragenden Elektronen in den jeweiligen Elektroden.

\subsubsection{Brinkman-Modell}

In diesem Abschnitt wird kurz das für die Auswertung relevante Brinkman-Modell eingeführt. Dabei werden einige wichtige Annahmen des Modells unter dem Blickwinkel der mathematischen Anwendung dargestellt.

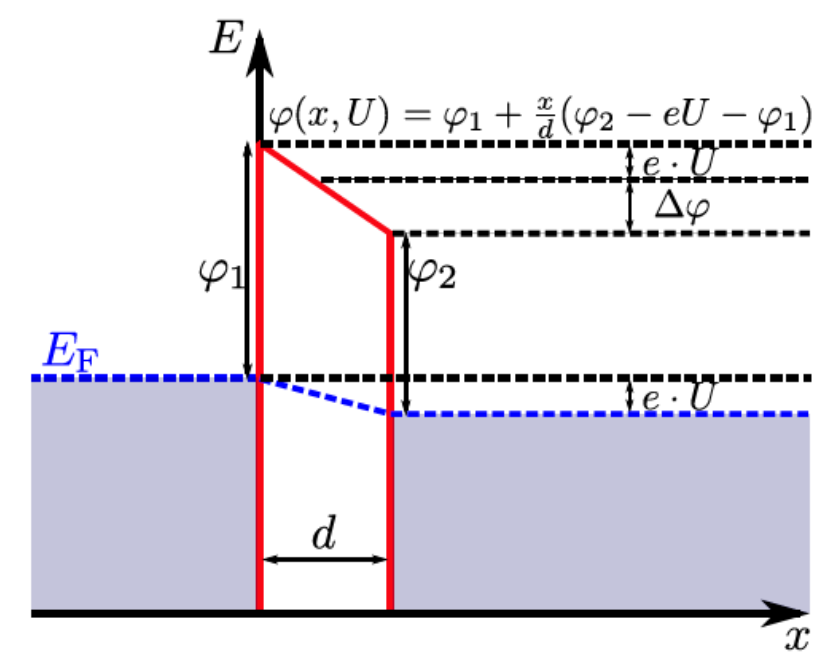

Abbildung 2.3.: Brinkmann-Modell einer trapezförmigen Potenzialbarriere [15].

Die Tunnelbarriere der magnetischen Tunnelelemente erweist sich als essentieller Parameter beim Spintransport sowohl für TMR-Effekt als auch für den Spintransfer torque (kurz STT). Zur Einschätzung der Qualität der Barriere kann auf das Brinkman-Modell zurückgegriffen werden. 
Wird ein Tunnelelement betrachtet, so kann es vereinfacht als zwei metallische Elektroden, getrennt von einer Potentialbarriere, dargestellt werden. Wird die Spannung an die Elektroden angelegt, so entsteht ein Elektronenfluss von einer zu der anderen Elektrode. Die Vereinfachung des Brinkman-Modells liegt daran, dass die Potentialbarriere der isolierenden Schicht als Trapezförmig angenommen wird (siehe Abbildung 2.3). Die Potenziale der beiden Elektroden stellen in diesem Fall die Seiten einer Trapez dar [22]. Diese Annahme erleichtert wesentlich die mathematischen Berechnungen und liefert trotz der Einfachheit gute Vergleichswerte.

Die Barriere selbst weist (unter der Annahme des trapezförmigen Verhaltens) für Potential $\varphi$ folgende Relation auf [22]:

$$
\varphi(x, U)=\varphi_{1}+\frac{x}{d}\left(\varphi_{2}-e U-\varphi_{1}\right)
$$

Die Transmissionswahrscheinlichkeit für ein Elektron, das einen Potential $V(x)$ durchläuft, ist nach [23]:

$$
T=\exp \left(-\frac{2}{\hbar} \int_{0}^{d} \sqrt{2 m(V(x)-E) d x}\right)
$$

Aus den beiden Formeln lässt sich eine nach in [22] gemachten numerische Lösung für den von der Spannung abhängigen Stromfluss entwickeln:

$$
J(U)=3,16 \cdot 10^{10} \frac{\bar{\varphi}^{\frac{1}{2}}}{d} \exp \left(-1,025 \cdot \bar{\varphi}^{\frac{1}{2}} d\right) \cdot\left[U-\frac{A_{0} \Delta \varphi}{32 \bar{\varphi}^{\frac{3}{2}}} e U^{2}+\frac{3 A_{0}^{2}}{128 \bar{\varphi}} e^{2} U^{3}\right]
$$

wobei $A_{0}=\frac{4 d \sqrt{2 m}}{3 \hbar}$ ist. Bei der Auswertung werden entsprechende Werte in folgenden Einheiten eingesetzt: $\varphi$ in V, d in Ångström und J in A/ $\mathrm{cm}^{2}$ [15].

Mit Hilfe dieses Modells kann die Barrierenhöhe der magnetischen Tunnelelemente abgeschätzt werden. Mit dem Modell können die Barrieren-Eigenschaften unterschiedlicher Tunnelelemente miteinander verglichen werden. Bei sehr dünnen $(<1 \mathrm{~nm})$ Barrierendicken werden jedoch die Vorhersagen des Brinkman-Modells zunehmend ungenauer [15]. 


\subsubsection{Kohärentes Tunneln}
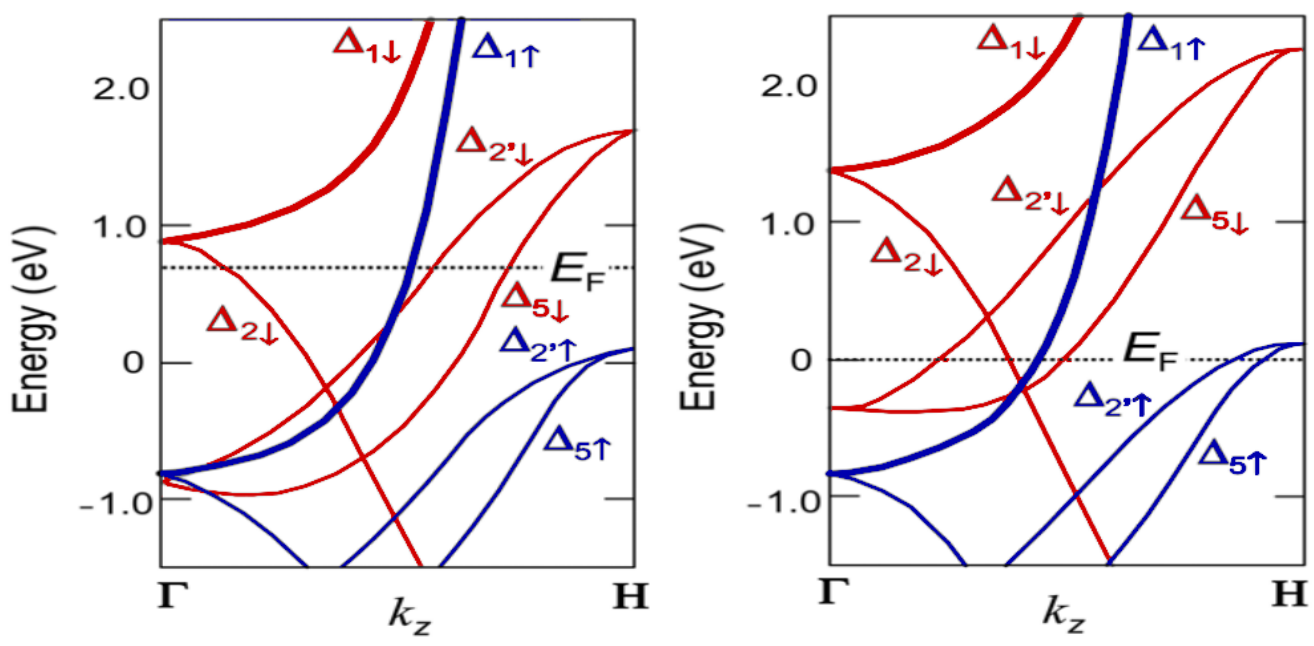

Abbildung 2.4.: Bandschema Co und Fe [20]. Blaue Linien stellen die Majoritätsbänder und die roten - die Minoritätsbänder dar. Die am Tunneln beteiligte Elektronen müssen Zustände am Fermi-Niveau haben.

In diesem Unterabschnitt wird der Effekt des kohärenten Tunnelns erläutert. Eine noch detailliertere Beschreibung befindet sich in den folgenden Quellen: [18, 15, 20, $16]$.

Die Tunnelbarriere einer MTJ kann amorph (z.B. Aluminiumoxid) oder kristallin (z.B. MgO) sein. Diese Eigenschaft der Barriere hat einen direkten Einfluss auf das Verhalten des gesamten Elementes. Im Falle einer amorphen Barriere können Elektronen aus dem Valenzband unabhängig von ihrer Symmetrie gleichwahrscheinlich von einer Elektrode in die andere tunneln. Für eine Spinpolarisation in solchen Tunnelelementen sorgt vor allem die Besetzung der Majoritäts- und Minoritätselektronen (siehe Abbildung 2.4). Betrachtet man zunächst den parallelen Zustand, so können nur die Elektronen tunneln, deren Energie $\leq E_{\text {Fermi }}$ ist. Für Co und Fe sind es $\Delta_{1}, \Delta_{2}, \Delta_{2^{\prime}}, \Delta_{5}$-Bänder. Die Majoritätsbänder sind an der Abbildung 2.4 in blau mit einem $\uparrow$ - Pfeil markiert, die Minoritätsbänder entsprechend in rot mit einem $\downarrow$ - Pfeil. Im parallelen Zustand haben alle diese Bänder (zumindest für Minoritätelektronen) Energiezustände an dem Fermi-Niveau und können deswegen beim Tunneln freie Zustände auf der anderen Elektrode finden. Betrachtet man dagegen den antiparallelen Fall, so könnten nur Elektronen aus den $\Delta_{2^{\prime} \uparrow \downarrow}, \Delta_{5 \uparrow \downarrow}$ - Bändern freie Zustände an der anderen Elektrode finden. Dies führt zu einer Absenkung des Stromes in dem AP-Zustand (bzw. zur Erhöhung des gemessenen Widerstandes). 

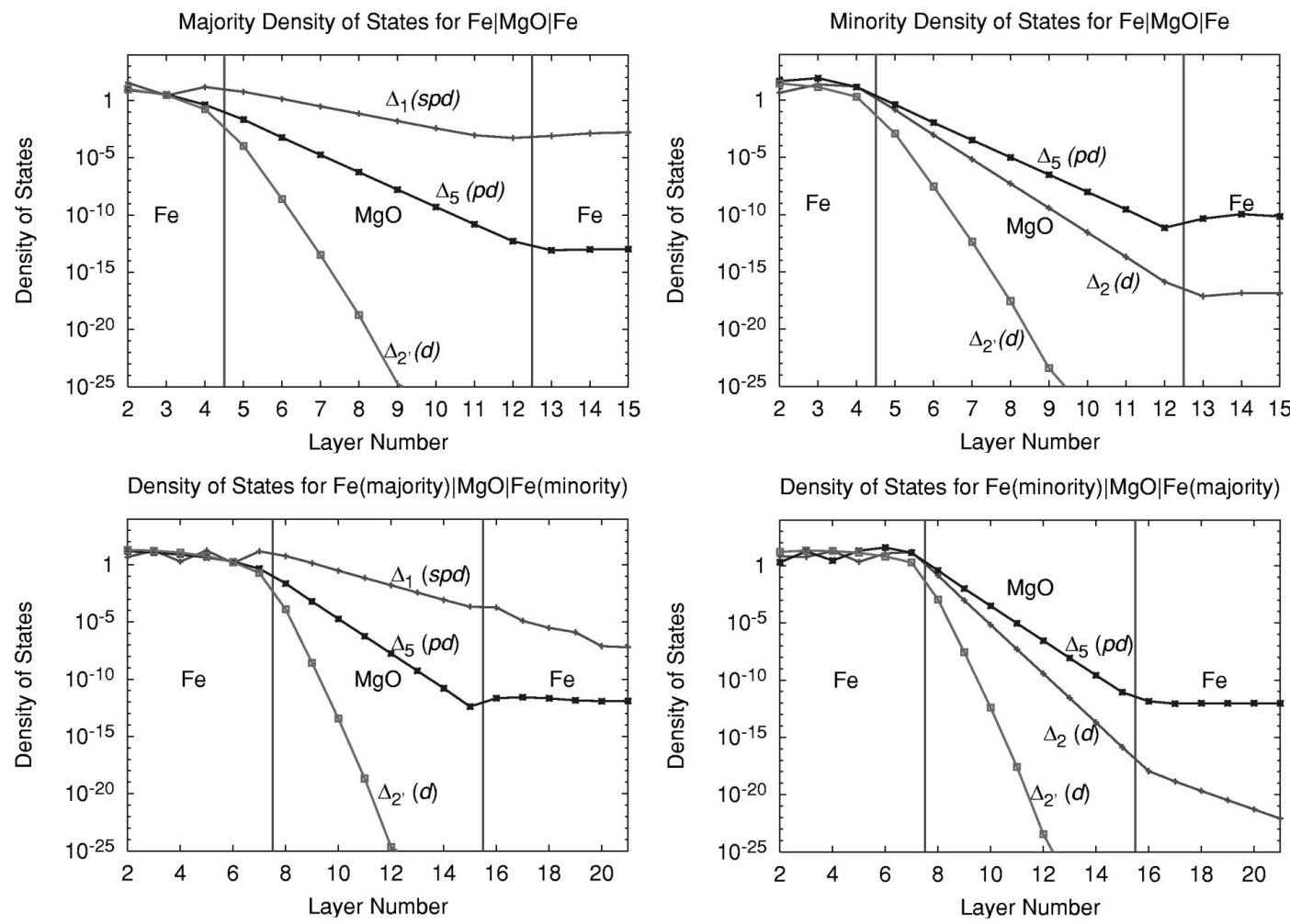

Abbildung 2.5.: Tunnelwahrscheinlichkeiten der Elektronen aus unterschiedlichen Energiebändern durch eine $\mathrm{MgO}$-Barriere [24]. Es wird von einem Elektronentransport von der linken zur rechten Elektrode ausgegangen.

Eine deutlich stärkere Erhöhung des Widerstandes im AP-Fall gegenüber dem P-Zustand, kann durch Verwendung einer kristallinen Barriere erreicht werden. Eine Besonderheit der $\mathrm{CoFeB} / \mathrm{MgO}$-basierten Tunnelelemente ist die hohe Gitteran-

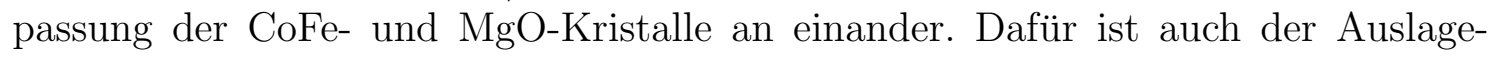
rungsschritt bei der Probenherstellung essentiell, denn $\mathrm{CoFeB}$ wächst beim Sputtern zunächst amorph auf und kristallisiert erst während der Auslagerung im Idealfall ausgehend von der MgO-Grenzfläche (siehe Unterabschnitt 3.1.3). Durch die Gitteranpassung der CoFe und MgO-Schichten wird die Barriere zu einem Spinfilter [24]. An der Abbildung 2.5 sind die Tunnelwahrscheinlichkeiten der Elektronen aus unterschiedlichen Energiebändern abgebildet. Betrachtet man die entsprechenden Tunnelwahrscheinlichkeiten für die nah an der Fermi-Energie liegende Bänder, so wird schnell klar, dass die $\Delta_{1}$-Bänder eine um mehrere Größenordnungen höhere Tunnelwahrscheinlichkeit als alle anderen Bänder aufweisen. Der Ursprung für diesen Effekt liegt in den Symmetrie-Eigenschaften der $\triangle$-Bänder (siehe Abbildung 2.6). Zusätzlich ist die Spinpolarisation des $\Delta_{1}$-Bandes gleich $100 \%$ (im AP-Zustand ist wegen der nicht vorhandenen freien Zustände auf der anderen Elektrode kein Tunneln möglich). Die Bänder mit freien Zuständen auf der anderen Elektrode (insbesondere $\left.\Delta_{2^{\prime} \uparrow \downarrow}, \Delta_{5 \uparrow \downarrow}\right)$, haben nach Abbildung 2.5 deutliche niedrigere Tunnelwahrscheinlichkeiten aufgrund der Spinfiltereigenschaften der MgO-Barriere. Dieser Effekt wird 

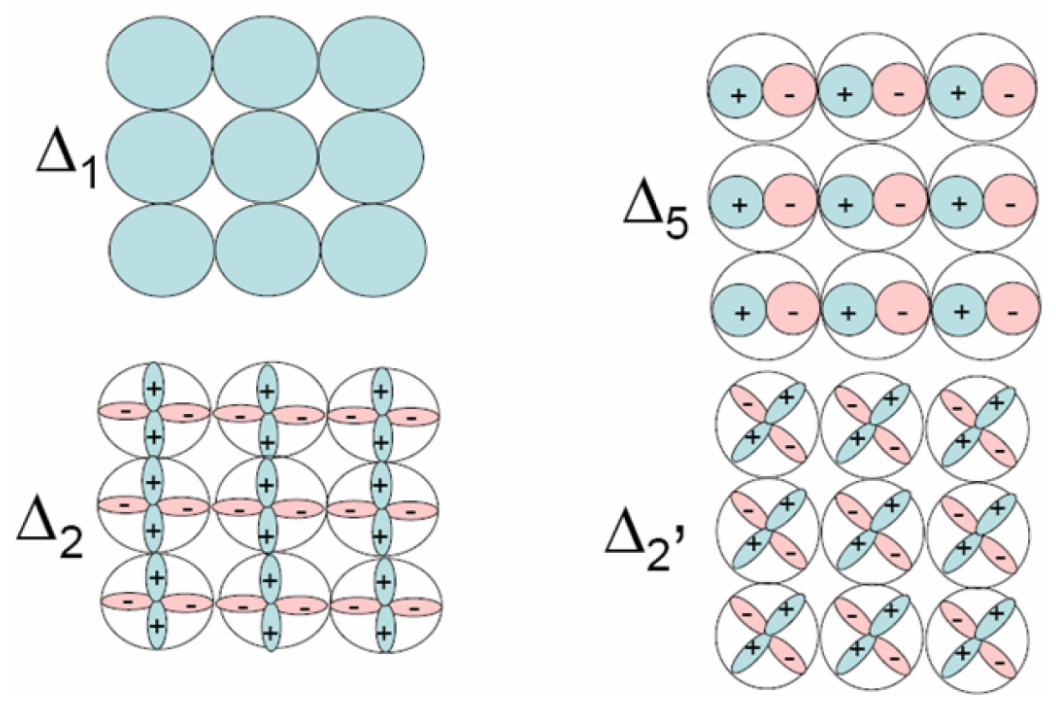

Abbildung 2.6.: Symmetrien der Bloch-Zustände der $\triangle_{1}, \triangle_{2}, \triangle_{2^{\prime}}, \triangle_{5}$-Bänder. $\triangle_{1}$ - Band besitzt eine kugelförmige Symmetrie und hat dadurch die höchste Tunnelwahrscheinlichkeit [24].

als kohärentes Tunneln bezeichnet (zur Veranschaulichung siehe Abbildung 2.7)
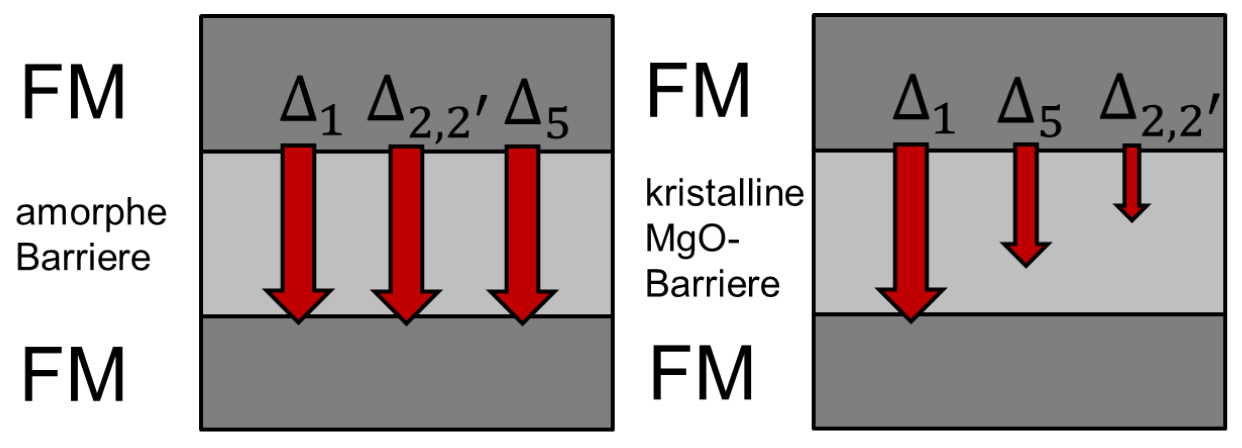

Abbildung 2.7.: Veranschaulichung des kohärenten Tunnelns. Für eine kristalline Barriere ist die Anzahl der tunnelnden Elektronen von dem Energieband abhängig. Die Barriere wird zu einem Spinfilter.

\subsection{Magnetische Anisotropie}

In vielen Materialien verhält sich die Magnetisierung ohne äußere Einflusse unterschiedlich für verschiedene Richtungen des Materials. Die Richtung, die von der Magnetisierung bevorzugt wird, also den Zustand der niedrigeren Energie bildet, wird als leichte Achse bezeichnet. Die magnetische Ausrichtung mit dem höchsten Energieaufwand wird als schwere Achse bezeichnet. 


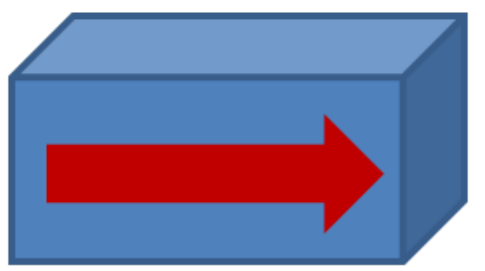

Formanisotropie

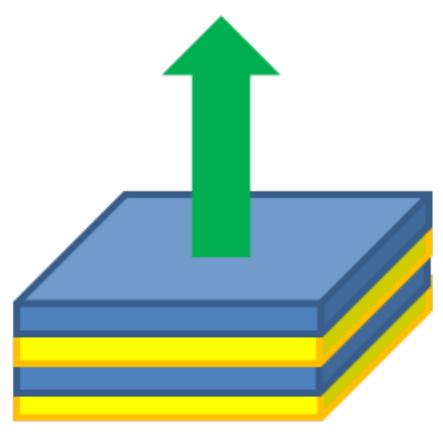

Magnetokristalline Anisotropie

Abbildung 2.8.: Skizze Anisotropie: (links) Formanisotropiebeitrag und (rechts) der Beitrag der kristallinen Anisotropie in einem Schichtenstappel mit dünnen Schichten.

Magnetische Anisotropie ist definiert als Energie, die notwendig ist, um die Magnetisierung aus der leichten zur harten Achse zu drehen [19].

Für die magnetische Tunnelelemente sind insbesondere folgende Anisotropiebeiträge wichtig: Die Volumenanisotropie $K_{V}$, und die Grenzflächenanisotropie $K_{i}$ und der Beitrag der Formanisotropie $\left(2 \pi M_{S}^{2}\right)$. Die Gesamtanisotropie $K_{\text {eff }}$ hat dann die Form $[25,26,27,28]$ :

$$
K_{\mathrm{eff}}=K_{V}-2 \pi M_{S}^{2}+\frac{K_{i}}{t}
$$

oder

$$
K_{\mathrm{eff}}=K_{V}-\frac{M_{S}^{2}}{2 \mu_{0}}+\frac{K_{i}}{t}
$$

Der Term $\frac{M_{S}^{2}}{2 \mu_{0}}$ entspricht der Demagnetisierung der Schicht (oder Streufeldenergie, die durch die Geometrie der ferromagnetischen Schicht beeinflusst wird, also proportional zur Formanisotropie ist), $t$ ist die Dicke des Ferromagneten. Diese zwei Formeln sind äquivalent und unterscheiden sich nur durch die für $M_{S}$ verwendete Einheiten (die Gleichung 2.6 wird für [T] und die Gleichung 2.7 wird für [emu/cc] verwendet).

Betrachtet man eine ferromagnetische Schicht, so unterscheidet sich die zum Sättigen der Magnetisierung benötigte Energie für unterschiedliche Richtungen abhängig von der Kristallstruktur (siehe Abbildung 2.9). In die $\langle 100\rangle$ Richtung haben 

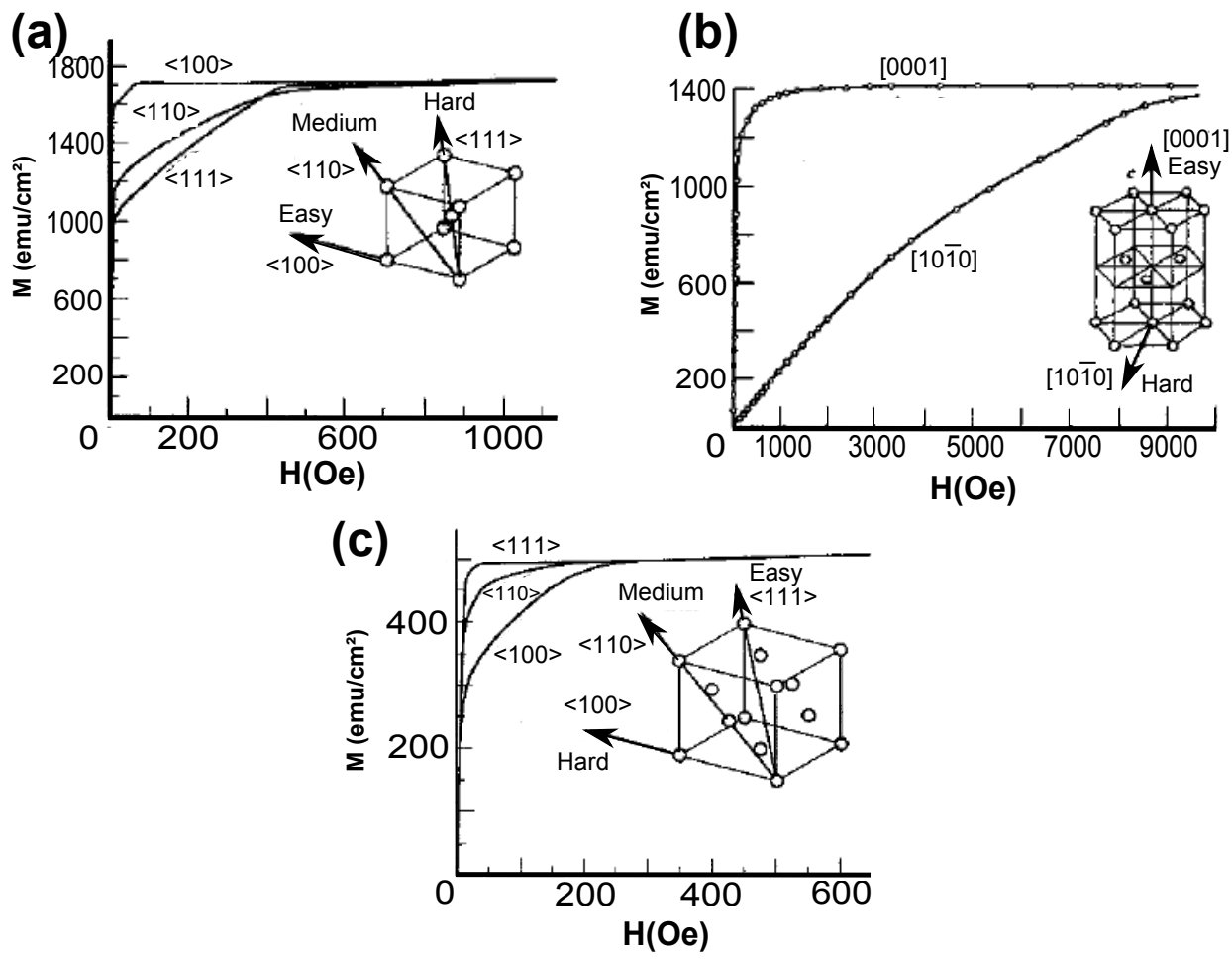

Abbildung 2.9.: Anisotropie-Skizze: (a) Fe, (b) Co und (c) Ni. [29]

Co und Fe sowohl für entsprechend fcc- als auch für bcc-Kristallstruktur eine leichte magnetische Achse. In die $\langle 111\rangle$ Richtung liegt für beide Materialien eine schwere Achse. Die CoFeB-Legierung wächst zunächst amorph und kristallisiert während der Auslagerung als bcc-Struktur, für die die Anisotropie-Achsen analog zum Fe liegen. Dies ist ein weiterer wichtiger Grund warum es vom Vorteil für Anwendungen ist, wenn der CoFeB-Kristall an die MgO-Struktur $\langle 100\rangle$ angepasst aufwächst. Jedoch ist die Kristallstruktur in solchen Multischichtsystemen nicht der einzige bestimmende Faktor für magnetische Anisotropie.

An der Abbildung 2.8 sind die Beiträge zu den zwei konkurrierenden Anisotropierichtungen abgebildet: der Volumenbeitrag mit der Formanisotropie zusammen versuchen die leichte Achse in die in-plane Richtung zu drehen, während der Grenzflächenbeitrag für die senkrechte Anisotropie sorgt. Deswegen kommt die out-ofplane Anisotropie insbesondere bei sehr dünnen magnetischen Schichten vor, da der Volumen- und Formanisotropiebeitrag für dünne Schichten niedriger ausfällt. Mit wachsender CoFeB-Schichtdicke erhöht sich der Volumenbeitrag bis eine kritische Schichtdicke erreicht wird, bei der die Beiträge für in- und out-of-plane Anisotropie gleich werden. In diesem Fall ist die effektive Anisotropie $K_{\text {eff }}=0$.

Die Oberflächenanisotropie hat ihren Ursprung in der Hybridisierung der Fe 3d und $\mathrm{O} 2 \mathrm{p}$ Orbitale an der $\mathrm{CoFe} / \mathrm{MgO}$-Grenzfläche (siehe Abbildung 2.10). Für eine sehr scharfe $\mathrm{CoFe} / \mathrm{MgO}$ - Grenzfläche liegt die Anisotropie in der out-of-plane Richtung [30]. An der Abbildung sieht man mögliche Hybridisierungen für eine Metall- 

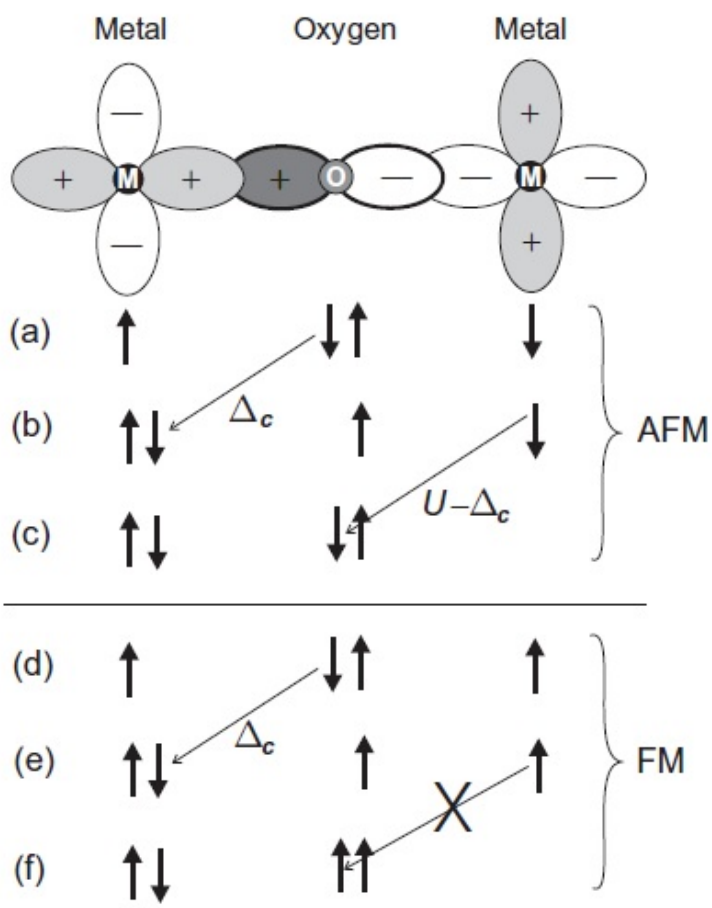

Abbildung 2.10.: Skizze der Hybridisierung. Die hellen Ovale entsprechen der negativen Wellenfunktion und die dunklen Ovale - der positiven Wellenfunktion. Die positive Wellenfunktion entspricht dem bindenden Orbital. In diesem Fall kommt es zu einer Hybridisierung der Orbitale [19].

Sauerstoff-Metall-Bindung. Für einen Ferromagneten entspricht (d) dem Grundzustand vor der Hybridisierung der Orbitale: Das Metall-Atom hat überschüssige ungepaarte Elektronen im d-Orbital und Sauerstoff hat gepaarte Elektronen im pOrbital. Die dunkleren positiven Ovale symbolisieren die positive (also bindende) Wellenfunktion und die hellen negativen Ovale entsprechen der negativen (also antibindenden) Wellenfunktion ${ }^{1}$. Bei der Hybridisierung kann also das gepaarte Elektron aus dem p-Orbital mit dem d-Orbital für positive Wellenfunktionen überlappen (siehe Abbildung 2.10 (e)). Die Hybridisierung ändert die Symmetrie der Orbitale, was eine Änderung in der Anisotropieenergie als Folge hat. Für CoFeB führt es zur out-of-plane Anisotropie [30].

Der davor diskutierte hohe Beitrag der $\mathrm{CoFe} / \mathrm{MgO}$ - Grenzfläche zur PMA (engl. Perpendicular Magnetic Anisotropy) kann jedoch abhängig von der Grenzflächenqualität abweichen. In Abbildung 2.11 sind drei Fälle für Grenzschichten mit unterschiedlichen Eigenschaften abgebildet: (a) entspricht dem bereits erwähnten „reinen“ Fall, bei dem Eisen und Sauerstoff miteinander nicht vermischte Schichten bilden.

${ }^{1}$ Da die positive Wellenfunktion nur in eine Richtung zeigt, bedeutet übertragen auf das $\mathrm{CoFe} / \mathrm{MgO}$-System: Sauerstoff hybridisiert an der Grenzfläche nur mit Eisen, nicht aber mit $\mathrm{Mg}$. 

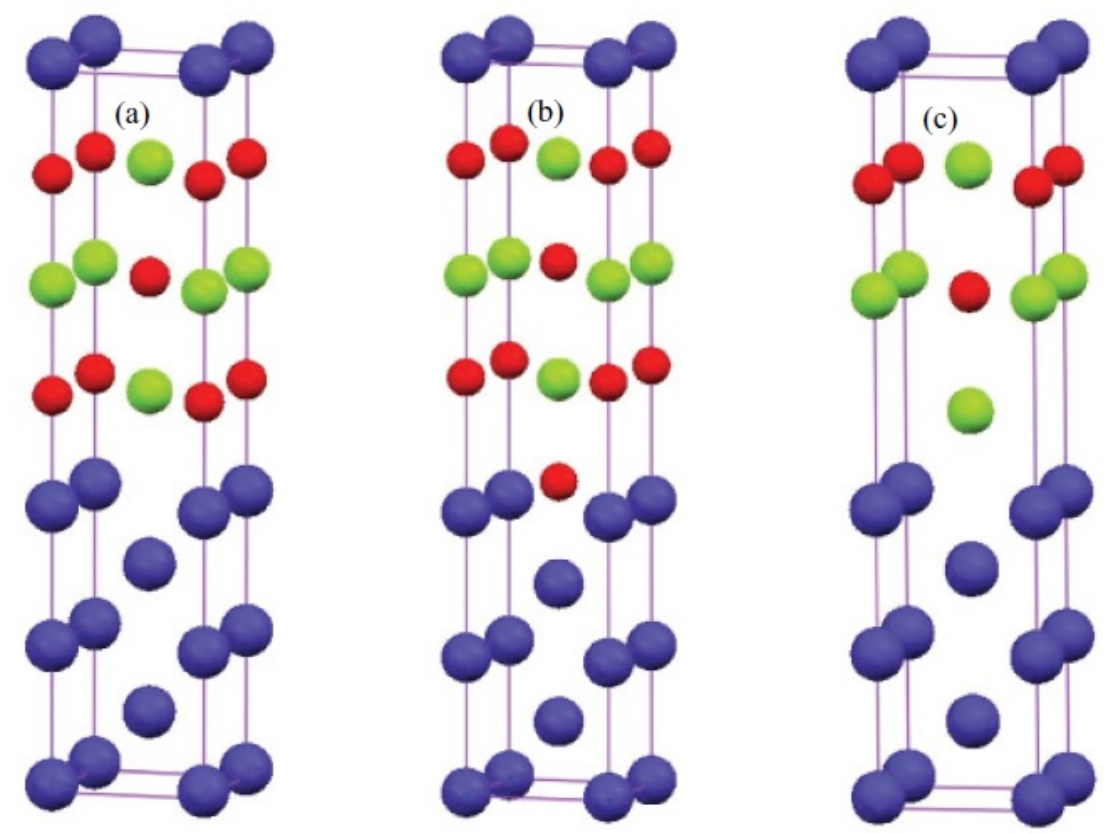

Abbildung 2.11.: Einfluss der Fe/MgO-Grenzfläche auf die PMA [30]: (a) Eisen (blau) und Sauerstoff (rot) bilden keine vermischte Schichten (reiner Fall), (b) Sauerstoff dringt in die Eisenschicht ein (Überoxidierung) und (c) entsprechend die Unteroxidierung, bei der PMA am niedrigsten liegt.

Bei (b) ist der sog. Fall der „Überoxidierung“ abgebildet, bei dem Sauerstoff in die Eisenschicht eindringt und die Kristallgeometrie entsprechend ändert. Dies hat eine Verringerung der PMA zur Folge. Entsprechend ist der letzte Fall (c) die „Unteroxidierung“" Die Fe-O Hybridisierung ist unterrepräsentiert und PMA ist kleiner im Vergleich zum Fall (a), liegt jedoch in der gleichen Größenordnung. Von Yang et al. [30] wurden in $\left[\mathrm{erg} / \mathrm{cm}^{2}\right]$ folgende Werte gemessen: $\mathrm{PM} A_{(a)}=2,93, \mathrm{PM}_{(b)}=2,27$ und $\operatorname{PM}_{(c)}=0,98$.

\subsection{Stoner-Wohlfahrth-Modell}

Zur Beschreibung der magnetischen Anisotropie eines Ferromagneten wird oft das Stoner-Wohlfahrth-Modell verwendet. Das Modell macht sehr gute Vorhersagen trotz seiner Einfachheit. Die Hauptannahme des Stoner-Wohlfahrth-Modells ist: der gesamte Bereich des Ferromagneten, der von Interesse ist, wird als eine einzige Domäne bzw. als sogenannter Stoner-Wohlfahrth-Partikel behandelt, der die Form eines Ellipsoids hat und magnetisiert ist. Mit dieser Annahme wird die Energiedichte $E_{\text {tot }}$ aus zwei Termen bestehen (siehe Gleichung 2.8): Anisotropieenergie $E_{A}$ und die Zeeman-Energie $E_{Z}=-M \cdot H$, mit $\mathrm{M}$ - magnetisches Moment eines Korns (bzw. der Domäne) und $\mathrm{H}$ - externes Magnetfeld [31]. Der Term $E_{A}$ beinhaltet alle Beiträge 
zur Anisotropieenergie (sowie Formanisotropie, Oberflächenanisotropie, Volumenanisotropie).

$$
E_{\text {tot }}=E_{A}+E_{Z}=K_{\text {eff }} \sin ^{2} \theta-H M \cos (\theta-\phi)
$$

Dabei beschreibt $\theta$ den Winkel zwischen $M$ und den Anisotropie-Achsen (normalerweise benutzt man als Konvention die leichte Achse), $\phi$ ist der Winkel zwischen angelegtem Feld $H$ und der leichten Achse $^{2}$.

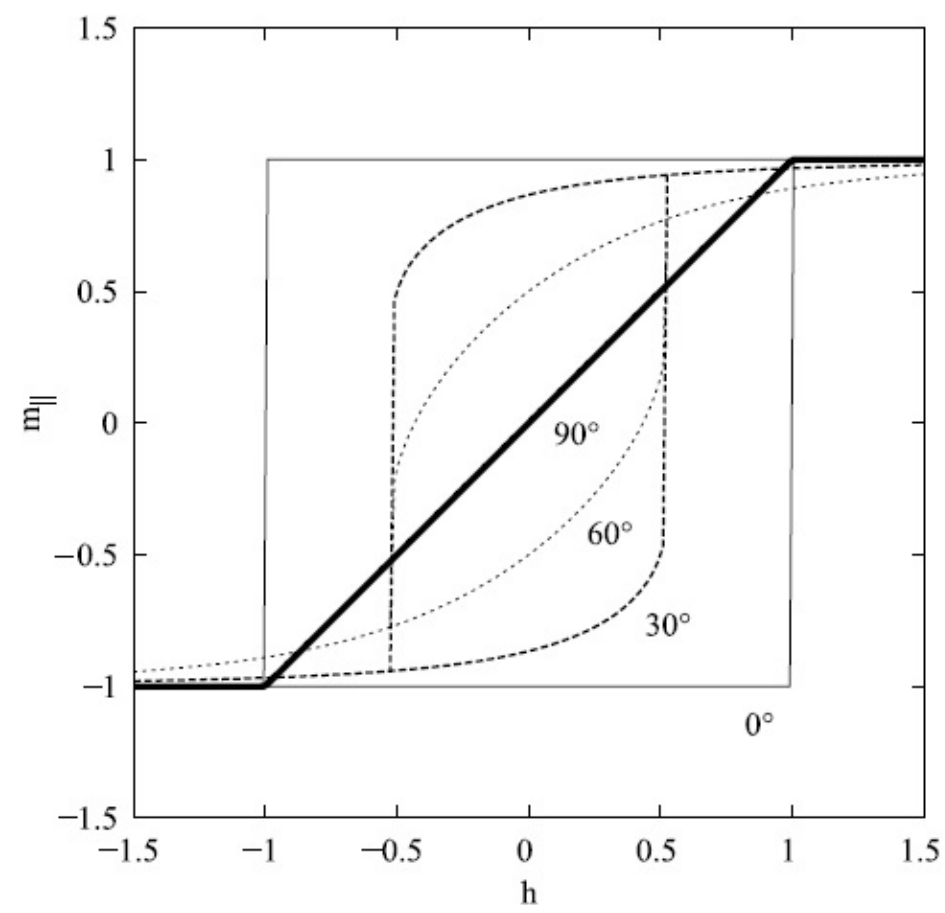

Abbildung 2.12.: Das Verhalten der Hysterese-Kurven in Abhängigkeit von $\phi$, der den Winkel zwischen dem angelegtem äußeren Feld und der leichten Achse beschreibt. Die x-Achse ist mit $h=\frac{H}{H_{K}}$ normiert (mit $H_{K}$, dem Anisotropiefeld). Die fett angezeichnete Kurve enspricht der schweren Achse [31].

Damit die Anisotropieenergie minimal wird, muss $\theta=0$ sein. Mit anderen Worten: $\theta$ definiert die Richtung der leichten Achsen, die bei den Minima der Funktion $E_{\text {tot }}$ liegen müssen (es kann mehrere Minima geben, die mehrere Achsen definieren würden). Betrachtet man das magnetische Verhalten des Stoner-Wohlfarth-Partikels abhängig von der Richtung des angelegten Feldes (also vom Winkel $\phi$ ), so ergibt sich eine Abhängigkeit, die in Abbildung 2.12 dargestellt ist [31]. Mit $m_{\|}=\cos (\theta-\phi)$ ist die Projektion $M$ auf $H$ gemeint. In Abbildung lässt sich erkennen, dass je stärker

\footnotetext{
${ }^{2}$ Durch Anlegen des Winkels $\theta$ in die andere Ebene, lässt sich relativ leicht aus diesem einfachen
} 2D-Modell ein 3D-Modell machen. 
die externe Feldrichtung von der Richtung der leichten Achse abweicht desto kleiner wird der hysteretische Beitrag (von der Hysterese eingeschlossene Fläche). Für $\phi=0^{\circ}$ und $\phi=90^{\circ}$ sind entsprechend die Hysteresen für die leichte und schwere Achsen abgebildet.

Das Stoner-Wohlfarth-Modell macht korrekte Aussagen zum Gesamtverhalten der magnetischen Schichten und beschreibt sehr gut durch seine Einfachheit einige magnetische Phänomene. Auch experimentell ist es vom großen Nutzen, da das Verhalten der Hysteresen damit erklärt werden kann. Das Modell stößt jedoch an seine Grenzen, wo die Existenz mehrerer magnetischen Domänen von großer Bedeutung ist. Bei der Untersuchung der Multischichtsysteme kann das Stoner-WohlfarthModell die Wechselwirkung zwischen den Schichten nicht berücksichtigen [31] und müsste dafür modifiziert werden (z. B. durch die Aufspaltung des Terms $E_{A}$ in alle Beiträge zur Anisotropieenergie). Für die Untersuchung der ferromagnetischen Einzelschichten und Diskussion der daran aufgenommenen Hysterese-Kurven eignet sich das Modell sehr gut und wird bei der Auswertung der gemessenen Hysterese-Kurven verwendet.

\subsection{Spin-transfer torque}

\subsubsection{Mechanismus des Stromschaltens}

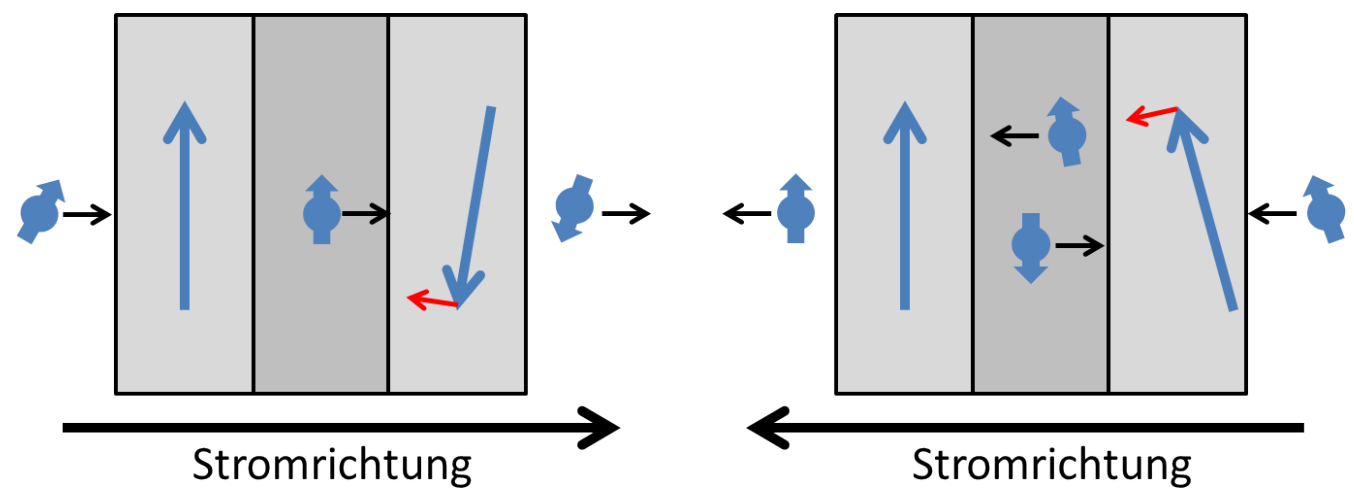

Abbildung 2.13.: Spin-transfer torque (kurz STT): (links) Skizze des Übergangs von AP- zu P-Zustand, (rechts) der P- zu AP-Übergang.

Passiert ein Elektron eine magnetisierte ferromagnetische Schicht, so wird es durch die Magnetisierung der Schicht spinpolarisiert [32]. Das Spinmoment der Elektronen wechselwirkt mit der Magnetisierung des Ferromagneten und kann sie somit beeinflussen [32, 33]. Bei einem magnetischen Tunnelelement kann dieser Effekt vereinfacht folgendermaßen beschrieben werden (siehe Abbildung 2.13): Nimmt man an, dass eine ferromagnetische Schicht eine feste Magnetisierung hat und die andere Schicht ihre Magnetisierung ändern kann, so wird zwischen antiparalleler 
(Abbildung 2.13 (links)) und paralleler (Abbildung 2.13 (rechts)) Ausrichtung der Magnetisation der Ferromagneten unterschieden. In Abbildung 2.13 wird die linke Elektrode als fest gesetzt. In dem AP-Zustand würden die Elektronen mit einem zufällig orientierten Spin in die Elektrode fließen. Je nach Orientierung der Spins können sie entweder durch das sp-Leitungsband der Majoritätselektronen oder durch das d-Leitungsband der Minoritätselektroden transportiert werden. In beiden Fällen übernehmen sie die Spinausrichtung der Elektrode. Aufgrund der hohen Spinpolarisation beim Elektronentransport der magnetischen Tunnelelmente werden die meisten Minoritätselektronen bei der zweiten Elektrode nicht ankommen (siehe Unterabschnitt 2.2.3). Die Majoritätselektronen können auf die andere Elektrode tunneln und durch das Drehmoment die Magnetisierung der zweiten Elektrode verändern. Vor allem wird ab einer kritischen Anzahl der tunnelnden Elektronen das Umschalten der freien Elektrode in den P-Zustand erwartet [33, 32]. Um das magnetische Tunnelelement aus dem P- in den AP-Zustand zurückzuschalten, muss die Stromrichtung umgeschaltet werden. In diesem Fall durchlaufen die Elektronen mit einem zufällig gerichteten Spin die freie Elektrode zuerst. Die Elektronen mit den zu der Elektrodenmagnetisierung gleich gerichteten Spins finden freie Zustände an der Elektrode. Die Elektronen mit den anders gerichteten Spins können ihre Spinausrichtung an die Elektrode anpassen, wobei es bereits zu einer Wechselwirkung zwischen der Elektrodenmagnetisierung und den Elektronenspins kommt, so dass bereits ein Drehmoment entsteht. Elektronen, die auf die zweite Elektrode tunneln, werden je nach ihrem Spin entweder von dem linken FM aufgenommen oder, falls ihre Spinausrichtung antiparallel zu der Magnetisierung des linken FM steht, zurückreflektiert. Die reflektierten Elektronen erhöhen den Beitrag des Drehmoments, welcher die Magnetisierung der zweiten Elektrode ändert. Ab einer kritischen Stromstärke klappt die Magnetisierung des zweiten Ferromagneten um.

\subsubsection{STT an magnetischen Tunnelelementen mit PMA}

Die zum Schalten der Schichten benötigte kritische Ströme $I_{c}$ erweisen sich als empfindlich gegenüber der magnetischen Anisotropie. Die Berechnungen von Khvalkovskiy et al. [14] zeigen unterschiedliche Erwartungen für die kritischen Ströme der Tunnelelemente mit in- und out-of-plane Anisotropie:

Für PMA wird die kritische Stromstärke folgendermaßen berechnet [14, 34]:

$$
I_{c}=\left[\frac{4 e k_{B} T}{\hbar}\right] \cdot \frac{\alpha}{\eta} \cdot \triangle
$$

Für in-plane Anisotropie (kurz IPA) [14]:

$$
I_{c}=\left[\frac{4 e k_{B} T}{\hbar}\right] \cdot \frac{\alpha}{\eta} \cdot \triangle\left(1+\frac{4 \pi M_{\mathrm{eff}}}{2 H_{k}}\right),
$$


mit $\eta=\frac{2 P}{1+P^{2}}, \alpha \sim 0,001-0,01-$ die Dämpfungskonstante, $\triangle=\frac{E_{A} V}{k_{B} T}-$ die thermische Stabilität $\left(E_{A}-\right.$ magnetische Anisotropieenergie, $V$ - Volumen der Zelle), $4 \pi M_{\text {eff }}$ - das effektive Entmagnetisierungsfeld, $H_{K}$ - Anisotropiefeld. Man erkennt leicht, dass die beiden Formeln sich nur um den Term $\frac{2 \pi M_{e f f}}{H_{k}}$ unterscheiden. Dies deutet auf einen höheren Schaltstrom bei einer in-plane Anisotropie hin. Khvalkovskiy et al. erwarten um den Faktor $\leq 1,5$ höhere Schaltströme für IPA-Elemente [14]. Diese Erkenntnis ist von großer Wichtigkeit für Konzipierung der Anwendungen. Je niedriger die benötigte Schaltströme werden, desto niedriger ist der Energieverbrauch in einem auf STT-basiertem Gerät. In dieser Arbeit werden in diesem Sinne die Schichtstapel mit senkrechter Anisotropie verwendet.

Ein weiterer wichtiger Parameter für die Anwendungen der STT-basierten Elemente ist die thermische Stabilität $\triangle$, die einen Maß für die durchschnittliche Zustandserhaltung der Elemente darstellt. E. Chen et al. [35] haben als Grenzwert $\triangle>66,6$ für Anwendungen vorgeschlagen ${ }^{3}$. Dieser Grenzwert besagt, dass ein Bit mit einer höheren thermischen Stabilität als $\triangle=66,6$ mindestens 10 Jahre lang permanent in $10 \mathrm{~ns}$ Abständen bei $\leq 80^{\circ} \mathrm{C}$ gelesen und beschrieben werden kann, ohne dass dabei Fehler auftreten [35]. Dieses Kriterium setzt bereits relativ strenge Anforderungen an die in der Technik verwendete STT-Bits und übersteigt den Rahmen einer Untersuchung der ersten in unserer Arbeitsgruppe hergestellten STTTunnelelemente. Der Wert $\triangle \approx 67$ stellt jedoch einen guten Vergleichswert für eine Diskussion dar.

\subsection{Dynamik an den MTJs}

Ein ferromagnetisches Material kann die Anregungsenergie auf drei Arten speichern (siehe Abbildung 2.14): als Energie der Elektronen, als Anregung der Phononen und als Änderung der magnetischen Ordnung. Die Untersuchung der ferromagnetischen Materialien auf ultrakurzen Zeitskalen im Femtosekundenbereich zeigen das Verhalten des magnetischen Moments der Ferromagneten. So wird nach einer kurzen Anregung die Magnetisierung der Schicht aus der Gleichgewichtslage (normalerweise entspricht sie der leichten Achse) ausgelenkt und versucht nach dem Abklingen des Anregungspulses die Gleichgewichtslage wieder zu erreichen. Dies geschieht in Form der Präzession der magnetischen Momente um die leichte Achse bis zum Erreichen des Gleichgewichts. Die Relaxationszeit für die Spin-Gitter-Kopplung für reine Ferromagneten wie Ni, Co und Fe liegt typischerweise im Bereich von $\tau_{s l} \sim 100 \mathrm{ps}$ (bei einer typischen Absorptionenergie im Bereich von $\mu \mathrm{eV}$ ) [19]. Für eine CoFeLegierung mit einer niedrigeren Anisotropie liegt die Relaxationszeit bei etwa 500 ps [19]. Mit anderen Worten: Je stärker die Anisotropie des Ferromagneten ist, desto kürzer ist die Relaxationszeit nach der Anregung.

Der Phonon-Elektron-Austausch hat charakteristische Energien im Bereich von einigen Millielektronenvolt. Die entsprechende Relaxationszeit liegt typischerweise bei etwa $\tau_{e l} \sim 1 \mathrm{ps}[36,19]$.

\footnotetext{
${ }^{3}$ Dieser Wert gilt für $1 \mathrm{MB}$ Datenmenge.
} 


\section{Elektronen Gitter}

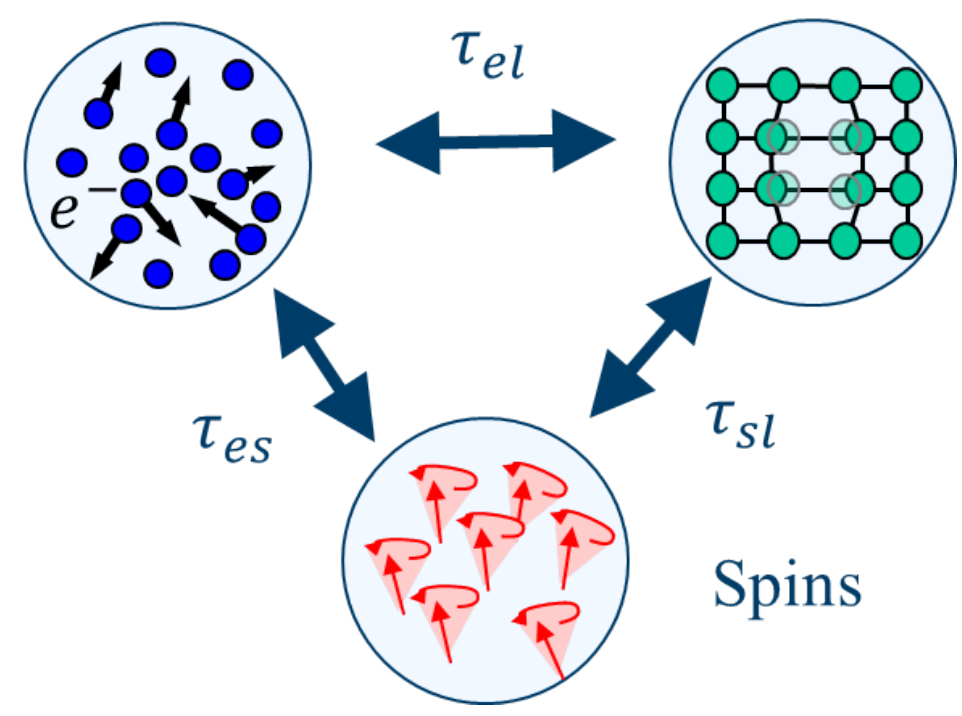

Abbildung 2.14.: Temperatur-Reservoire ferromagnetischer Materialien [17].

Der Energiebereich der Absorptionsenergie 1.5-3 eV entspricht der Elektron-SpinAnregung mit der Zeit ${ }^{4} \tau_{e s}$ unter 1 ps [19]. Dieser Energie- und Zeitbereich ist in dieser Arbeit von besonderem Interesse, weil sie unter dem Blickwinkel der Spintransportprozesse und deren Elektron-Spin-Anregungen untersucht werden sollen.

Die Entmagnetisierung auf ultrakurzen Zeitskalen ist von aktuellem Interesse, da der Mechanismus noch nicht für alle ferromagnetische Systeme vollständig geklärt ist. Die bereits durchgeführte Untersuchungen deuten auf Spintransort als Ursprung für die ultraschnelle Entmagnetisierung hin [38, 40, 37].

In den Arbeiten [38, 40, 37] wird gezeigt, dass ein Laserpuls die Elektronen in der ferromagnetischen Schicht anregen kann. Da in dem Ferromagneten die Energiebänder für Majoritäts- und Minoritätselektronen (bzw. für spin-up- und spindown-Elektronen) unterschiedlich gefüllt sind, kommt es bei der Anregung zu Sprüngen der Elektronen auf unterschiedliche Bänder oberhalb des Fermi-Niveaus (siehe Abbildung 2.15). Die Majoritätselektronen (spin-up) werden bei der Anregung auf das sp-Band angeregt (hohe Ausbreitungsgeschwindigkeit in dem Band und lange Lebensdauer [39]), während die Minoritätselektronen (spin-down) auf das d-Band gebracht werden (langsamere Ausbreitungsgewschwindigkeit, kürzere Lebensdauer [41]). Dies führt zu einer Spinpolarisation bei der Elektronenausbreitung in dem Ferromagneten. Kampfrath et al. [38] berichten über einen spinpolarisierten Stromfluss in Abhängigkeit von einer Buffer-Schicht auf Zeitskalen von $<300$ fs ( THz Spinstrompulse). Dabei werden je nach der Puffer-Schicht entweder die sp-Bänder (bei

\footnotetext{
${ }^{4}$ Die neuesten Untersuchungen zeigen sogar noch schnellere Effekte auf Zeitskalen von etwa 100$400 \mathrm{fs}[37,38,39]$.
} 


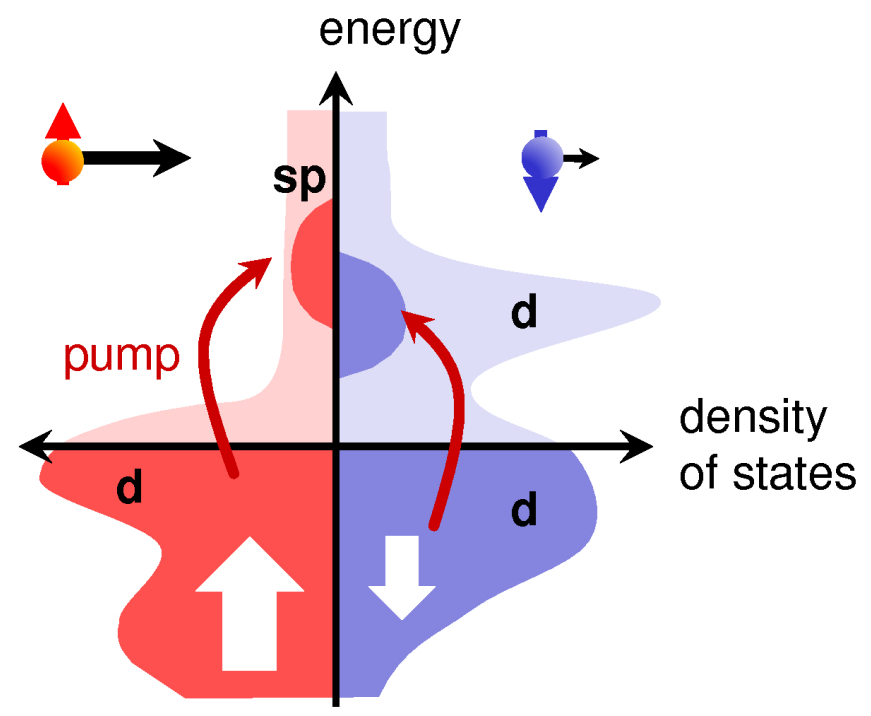

Abbildung 2.15.: Skizze des Übergangs bei der Elektronenanregung der ferromagnetischen Eisenschicht [38].

Gold) oder d-Bänder (bei Ruthenium) von Elektronen aus der Eisenschicht besetzt. Dies entspricht der $\uparrow$ und $\downarrow$ - Ausrichtung der Elektronen.

Werden die angeregten Elektronen (auch heiße Elektronen genannt) eines magnetischen Tunnelelements betrachtet, so können sich die Elektronen nicht frei durch die Barriere bewegen. Damit sie durch die Barriere tunneln können, muss die Tunnelwahrscheinlichkeit ausreichend hoch sein und die entsprechende Spinausrichtung gewährleistet sein (siehe Unterabschnitt 2.2.3). Werden die Elektronen bei der Anregung auf ein Energieniveau gebracht, das sowohl eine hohe Tunnelwahrscheinlichkeit als auch Zustände auf der zweiten Elektrode besitzt, so können diese Elektronen auf die zweite Elektrode tunneln. Dabei kann die Gesamtmagnetisierung erhöht oder erniedrigt werden, je nach dem welche Elektronen am Spintransport beteiligt sind (siehe Abbildung 2.16). He et al. [40] haben mit Laser-Pulsen angeregte magnetische Tunnelelemente untersucht. In Abbildung 2.16 ist ein von He et al. vorgeschlagenes Modell zur Erklärung der Entmagnetisierung auf ultrakurzen Zeitskalen dargestellt. Bei der Elektronenanregung im parallelen Zustand kommt es zum Transport zwischen den tunnelnden Majoritätselektronen der ersten Elektrode und den Majoritätselektronenzuständen der zweiten Elektrode (Abbildung 2.16 (a)). Im AP-Zustand können die Minoritätselektroden an dem Tunnelprozess teilnehmen (Abbildung 2.16 (b)), wodurch sich die Entmagnetisierungsstärken der Pund AP-Zustände unterscheiden müssten. Diesen Effekt konnten He et al. nachweisen $[40]$.

Das Tunneln der heißen Elektronen erlaubt eine Entmagnetisierung der magnetischen Tunnelelementen auf ultrakurzen Zeitskalen $<300$ fs, da die Driftgeschwindigkeit der Elektronen in einem Element je nach der Anregungsenergie bei ca. $0,1 \frac{\mathrm{nm}}{\mathrm{fs}}$ 


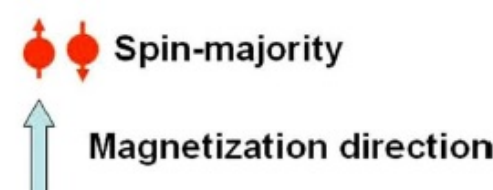

+. Hot electron

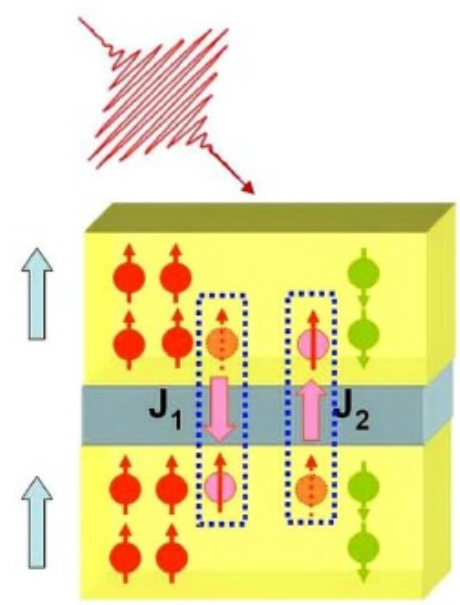

(a)
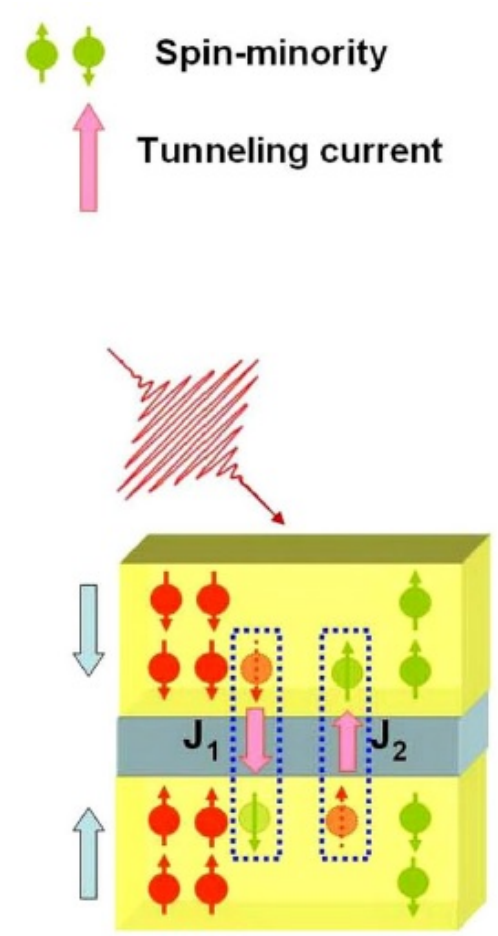

(b)

P state

AP state

Abbildung 2.16.: Tunneln der angeregten Elektronen in einem magnetischen Tunnelelement [40].

für Minoritätselektronen liegt (0,8 $\frac{n m}{f s}$ für Majoritätselektronen $\left.{ }^{5}\right)$ [39].

${ }^{5}$ Diese Werte wurden von Zhukov et al. [39] für dünne Eisenfilme gemessen. 



\section{Experimentelle Methoden}

In dieser Arbeit diskutierte Schichtsysteme wurden in einer Ultrahochvakuum-Anlage mittels Elektronenstrahlverdampfen und Sputtern hergestellt. Bei der Strukturierung der Proben wurden optische Lithographie und Elektronenstrahllithographie (engl. e-beam lithography) verwendet. Die Proben wurden elektrisch und optisch charakterisiert. Die für diese Arbeit relevante Schichtwachstums-, Strukturierungsund Charakterisierungsmethoden werden im Folgenden beschrieben.

\subsection{Probenpreparation}

\subsubsection{Substratreinigung}

In dieser Arbeit wurden Substrate aus MgO (100) und Silizium mit einer thermisch oxidierten Schicht von ca. $500 \mathrm{~nm}$ verwendet. Substrate wurden passend geschnitten (einer Probe entspricht eine Substratgröße von $1 \mathrm{~cm}^{2}$ ) und anschließend gereinigt.

Bei der Reinigung wurden Substrate in einem Becher mit Aceton zwei Minuten lang in einem Ultraschallbad geschwenkt. Danach wurden sie zwei Minuten lang in Isopropanol geschwenkt und anschließend mit einer Stickstoffpistole getrocknet.

Die Oberfläche der Substrate wurde anschließend mit einem Lichtmikroskop untersucht. Gegebenenfalls wurde die Prozedur wiederholt.

\subsubsection{Schichtenwachstum}

Nach der Reinigungsprozedur wird das Substrat auf einem Probenhalter befestigt. Anschließend wird der Probenhalter auf den Manipulator der Schleuse-Kammer angeschraubt, die den Zugang zu den anderen Kammern des im Folgenden vorgestellten UHV-Systems erlaubt.

Das gesamte in dieser Arbeit benutzte UHV-System besteht aus 4 Kammern: Schleuse-, Transfer-, Sputter- und Elektronenstrahlverdampfkammer (im Folgenden als Große Kammer bezeichnet). Alle diese Kammern werden separat abgepumpt, haben jedoch Zugangsventile, so dass der Probenhalter zwischen den Kammern im Ultrahochvakuum ( $p \sim 10^{-9} \ldots 10^{-10}$ mbar $)$ verfahren werden kann.

Das Schichtenwachstum kann in zwei Kammern durchgeführt werden: SputterKammer und der Großen Kammer. 


\subsubsection{Sputtern}

Das Wort Sputtern kommt vom englischen to sputter (auf deutsch zerstäuben). Diese Methode wird oft in der Industrie verwendet, um große Flächen mit Nanometer dicken Schichten homogen und reproduzierbar zu bedampfen.

Beim Sputtering benutzt man für jedes Material eine separate Sputterquelle. Es ist auch möglich Legierungen (z. B. CoFeB-Legierungen) und Moleküle (z. B. MgO) mit dieser Methode aufzuwachsen.

Eine Sputter-Anlage besteht in der Regel aus einer Vakuum-Kammer mit einer Sputterquelle, die wiederum aus einer Gleichspannungsversorgung, Kathode und Anode besteht. Durch präzise gesteuerte Ventile und Regler wird in die Kammer ein Edelgas mit einer vorgegebenen Flussgeschwindigkeit gepumpt. Sobald in der Kammer ausreichend viel Gas zur Verfügung steht (Arbeitsdruckbereich $p \sim 10^{-3}$ mbar) kann an die Sputterquelle Spannung gelegt werden. Dies hat zur Folge, dass aus der Kathode Elektronen austreten und zur Anode hin beschleunigt werden. Auf dem Weg zur Kathode können Elektronen die Edelgasatome (in unserem Fall Argon) ionisieren. Die positiv geladenen Ar-Ionen werden durch die negative Ladung der Kathode angezogen. Bei hinreichender Geschwindigkeit können Ar-Ionen durch Kollisionen Atome von der Kathode (Sputtertarget) lösen. Die so abgetragenen Target-Atome können sich auf dem Substrat absetzen und zum Schichtenwachstum beitragen [42].

Die Target-Atome werden fokussiert in einem Strahl senkrecht zur Target-Oberfläche in die Kammer gestreut. Dies wird durch die Wirkung der Magnetfelder gewährleistet (diese Methode wird auch Magnetronsputtering genannt [42]). Durch die Stöße miteinander und den Argon-Atomen wird der Strahl in der Nähe der Quelle leicht und weiter von der Quelle dann stärker defokusiert. Die Probe befindet sich in einem Abstand von ca. $3 \mathrm{~cm}$ bei dem der Strahl noch homogene und hohe Aufdampfrate liefert. Ist man jedoch an den Proben mit einer variablen Schichtdicke interessiert, so kann die Probe unter einem Winkel zu dem SputterTarget eingebaut werden. Durch den Winkel zwischen der Sputter-Quelle und der Probe (siehe Abbildung 3.1 (links)) wird ein Schichtdickengradient erzeugt. An der Abbildung ist eine Skizze der Sputter-Quelle mit der Position der Probe angezeichnet. Die Target-Atom-Ausbreitung wurde als kugelförmig von der SputterQuelle dargestellt. Eine Reihe von Testproben wurde hergestellt und deren CoFeBSchichtdickengradient wurde mit einem Profilometer vermessen. Das Ergebnis der Messungen ist als Skizze an Abbildung 3.1 (rechts) abgebildet. Geht man einfachheitshalber von zwei Gradientrichtungen aus, so ergeben sich zwei Achsen mit unterschiedlich hohen CoFeB-Schichten. Als $100 \%$ wird der Bereich bezeichnet, der mit der Schwingquarzanzeige komplett übereinstimmte (die Schwingquarzeinstellungen wurden für diesen Bereich angepasst). Von dem Bereich ausgehend stieg die Schichtdicke entlang der beiden Gradientachsen um entsprechend $20 \%$ und $30 \%$. Proben mit einem CoFeB-Keil ermöglichten eine schichtdickenabhängige Untersuchung der CoFeB-basierten dünnen Schichten. Dadurch ist eine sehr gute Vergleichbarkeit der Messungen gewährleistet, da es sich bei mehreren Messgängen um die gleiche (also identische Herstellungsparameter) Probe handelt. 

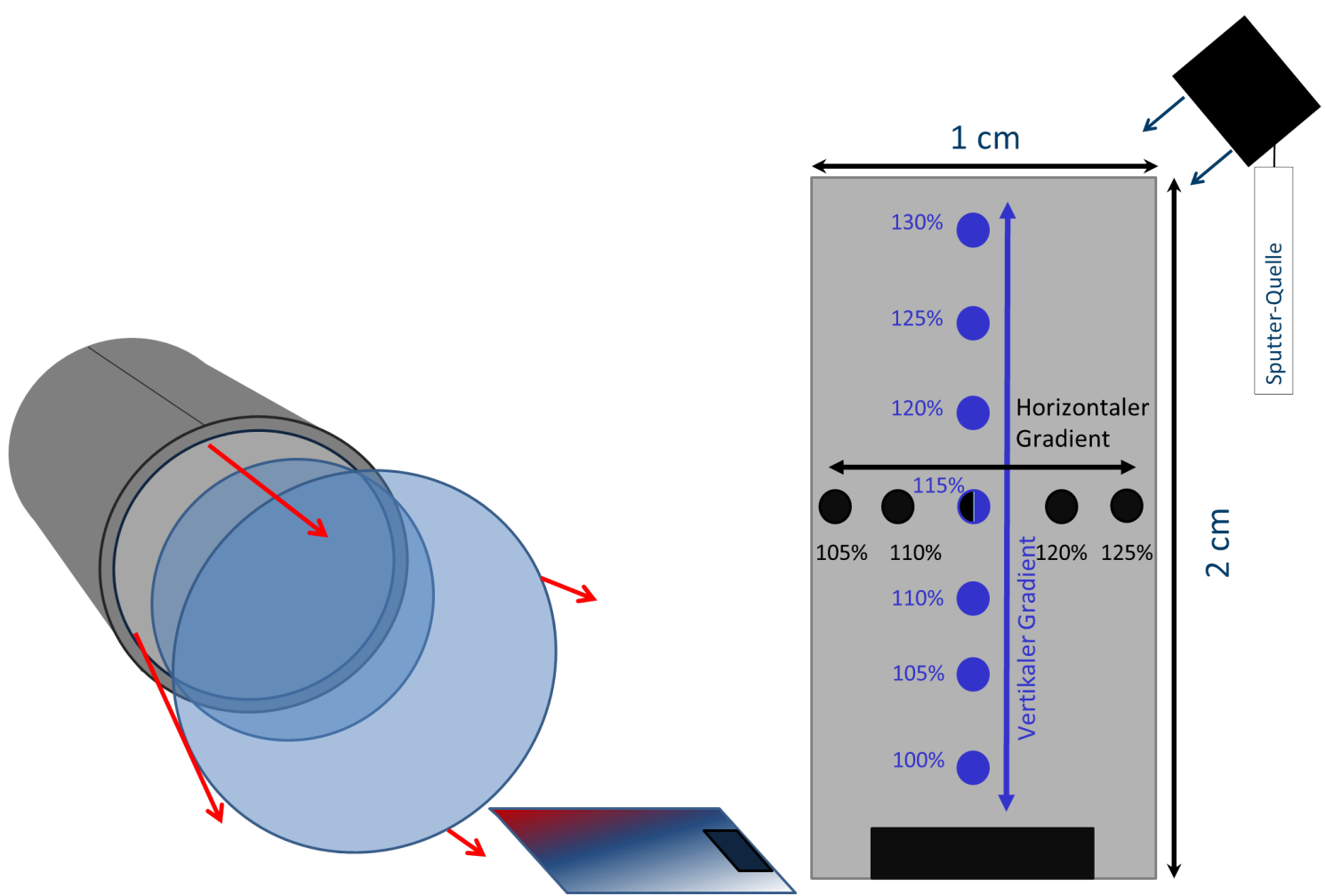

Abbildung 3.1.: Positionen der Probe bzgl. der CoFeB-Sputterquelle (links). Als roter Gradient ist der Bereich mit der höchsten Schichtdicke nach dem Aufdampfen markiert. Gemessene Stärke des CoFeB-Gradients. Als 100 \% wird der Bereich bezeichnet, auf den der Schwingquarz kalibriert wurde.

Zur Messung des Schichtdickengradients wurde folgendermaßen verfahren. Zunächst wurde eine Struktur entlang des Gradients aufgebracht. Dafür wurde das Substrat entweder mit Positiv-Lack oder das Substrat wurde mit einem Klebestreifen entlang des Gradients zugeklebt (die zweite Methode erwies sich als vergleichbar von der Genauigkeit jedoch deutlich zeitsparender, deswegen wurde für spätere Kalibrierung der Klebestreifen verwendet). Nach dem Aufdampfen von $100 \mathrm{~nm}$ CoFeB, wurde vom Substrat der Klebestreifen mit dem CoFeB darauf entfernt und die Probe wurde im Propanol gereinigt. Anschließend wurde mit dem Profilometer die Höhendifferenz zwischen beim Sputtern vom Streifen bedeckten (also ohne CoFeB-Schicht) und bedampften Flächen gemessen. Die gemittelten Ergebnisse sind in Abbildung 3.1 (rechts) zu sehen. Für die Schichtdicken zwischen 100 und $130 \mathrm{~nm}$ ergab sich eine Standardabweichung von ca. $\pm 3-4 \mathrm{~nm}$. Dies entspricht der Abweichung von $\sim 4 \%$ und ist mit der Genauigkeit des Profilometers vergleichbar.

\subsubsection{Elektronenstrahlverdampfen}

Das Elektronenstrahlverdampfen wird in der separaten Kammer durchgeführt. Sie besteht aus zwei Elektronenkanonen mit jeweils vier verfahrbaren Tiegeln. In den 
Tiegeln sind zu verdampfende Substanzen angebracht. Die Elektronenkanone besteht aus einer Glühkathode und einem Magneten, der von der Kathode emittierte Elektronen auf eine Kreisbahn lenkt, so dass das Material im Tiegel getroffen werden kann. Die thermische Leistung, die dabei auf das Material übertragen wird, wird durch die Einstellung der Stromstärke variiert. Durch eine Feinjustage der Stromstärke kann die Aufdampfrate verstellt werden. Die Aufdampfrate und aufgedampfte Schichtdicke wird mit Hilfe eines Schwingquarzes kontrolliert. Das Gerät kann Aufdampfraten von bis zu 0,01 $\AA$ reproduzierbar anzeigen $[18,15,16]$.

Der Druck in der Kammer ist ein wichtiger Parameter für die Qualität der Schichten. Je nach dem wie hoch der Restgasdruck in der Kammer ist, werden die chemische Homogenität und Oberflächenrauigkeit der Schichten beeinflusst. Ein Restgasdruck niedriger als $10^{-9}$ mbar ist für die Wachstumsgeschwindigkeiten der Schichten von einigen Minuten ausreichend [43]. In der Großen Kammer lag Druck im Bereich zwischen $\sim 10^{-9}$ und $10^{-10}$ mbar. Für eine zusätzliche Verringerung des Restgasdrucks wurde Tantal-Sublimation verwendet: aus dem Tantal Tiegel wird Material verdampft, so dass die Tantalatome mit dem Restgas in der Kammer reagieren können. Die entstehende Verbindungen werden von der Turbopumpe mit der Zeit (einige Minuten) aus der Kammer entfernt. Als Folge wird eine Druckabsenkung beobachtet [18].

\subsubsection{Auslagerung der Proben}

Eins der wichtigsten Bestandteile der in dieser Arbeitsgruppe hergestellten magnetischen Tunnelelemente (engl. Magnetic Tunnel Junctions, kurz MTJs) ist die EisenKobald-Bor-Legierung (kurz CoFeB), die ferromagnetische Eigenschaften zeigt und dadurch die Magnetisierung des gesamten Tunnelelements bestimmt wird. Nach dem Sputtern ist die CoFeB-Schicht amorph, jedoch für das Kohärente Tunneln (siehe Unterabschnitt 2.2.3) wird eine an die MgO-Barriere angepasste Struktur benötigt. Um die an MgO-Struktur angepasste Kristallisation der CoFeB-Legierung zu erreichen, wird nach dem Herstellen des Schichtensystems der sog. Auslagerungsschritt (engl. Annealing) gemacht. Dabei wird die Probe im Vakuum erhitzt und somit der Kristallisationsvorgang der $\mathrm{CoFeB}-\mathrm{Schicht}$ angeleitet. Dafür wird die Probe in die externe Auslagerungskammer gebracht. Das Vakuum in der Kammer liegt bei etwa $10^{-6}-10^{-7}$ mbar. In der Kammer kann die Probe innerhalb weniger Minuten auf eine Temperatur bis zu $600^{\circ} \mathrm{C}$ geheizt werden. Zusätzlich kann ein externes Magnetfeld bis $300 \mathrm{mT}$ angelegt werden.

Eine Probe wird nach dem Herstellen auf dem Probenhalter der Auslagerungskammer eingespannt. Anschließend wird die Luft aus der Kammer gepumpt. Der Probenhalter ist mit Heizelementen ausgerüstet und kann damit die Temperatur der Probe direkt erhöhen. Nachdem die gewünschte Temperatur erreicht ist, wird sie konstant gehalten (Schwankungen im Bereich $\pm 2{ }^{\circ} \mathrm{C}$ ). In der Regel wird die Probe 30-60 min ausgelagert. Die typische Auslagerungstemperatur unterscheidet sich für in- und out-of-plane Schichtstapel: $T_{P M A}=300^{\circ} \mathrm{C}$ und $T_{I P A}=450{ }^{\circ} \mathrm{C}$. Im Falle der dünneren PMA-Proben (engl. perpendicular magnetic anisotropy, kurz PMA) 
ist die niedrigere Auslagerungstemperatur von Vorteil, da sie bereits für eine Kristallisation der dünnen CoFeB-Schicht ausreichend ist, jedoch noch zu niedrig für negative Beeinflussung durch z. B. Tantaldiffusion ist [18]. Bei den dickeren IPAProben (in-plane magnetic anisotropy, kurz IPA) ist die Tantaldiffusion erst bei der Temperatur von etwa $550^{\circ} \mathrm{C}$ von Bedeutung. Die Auslagerungsdauer der beiden Probenarten beträgt eine Stunde.

\subsection{Strukturierung}

\subsubsection{Lithographie}

Die Probenstrukturierung erfolgt mittels optischer Lithographie. Dafür werden die vollflächig bedampfte Proben zunächst mit Aceton und Propanol gereinigt. Anschließend werden sie mit einer Stickstoffpistole getrocknet. Die Lösungsmittelreste werden beim zweiminütigen Ausbacken bei $85-100{ }^{\circ} \mathrm{C}$ auf einer Heizplatte verdampft [18].

Die gereinigte Proben werden an einen Spin-Coater angebracht und ca. $40 \mu \mathrm{l}$ vom Negativ-Lack AR-N 4340 werden auf die Probenoberfläche gebracht. Anschließend wird die Probe mit einer Rotationsfrequenz von $6000 \mathrm{~min}^{-1}$ rotiert, wodurch der Lack auf der Probenoberfläche gleichmäßig verteilt wird. Diese Rotationsfrequenz entspricht einer Photolackschichtdicke von 1,27 um [44].

Nach dem Belacken wird die Probe auf der Heizplatte zwei Minuten lang ausgebacken, um die Lösungsmittel, die im Lack vorhanden sind, zu verdampfen. Nachdem der Lack durch das Ausbacken ausgehärtet ist, wird die Probe belichtet. Dafür wird der Belichter MJB4 der Firma Süss MicroTec mit UV-Licht $(\lambda=320 \mathrm{~nm})$ verwendet. Die Probe wird in den Belichter unter eine entsprechende Maske eingebaut und 5 Sekunden lang mit UV-Licht bestrahlt. Anschließend wird die Probe das letzte Mal ausgebacken. Für optische Lithographie werden in der Regel Masken mit Strukturen verwendet, deren Kantenlängen oder Durchmesser $\geq 3 \mu \mathrm{m}$ sind, den kleinere Strukturen können an der Probestation nicht kontaktiert werden.

Die belackte und belichtete Probe muss entwickelt werden. Dafür wird der AR 300-47 Entwickler verwendet, in dem die Probe 75 Sekunden geschwenkt wird. Nach der Entwicklung bedeckt der ausgehärtete Lack nur die Flächen, die vom UV-Licht belichtet wurden. Die restliche Oberfläche der Probe bleibt vom Lack unbedeckt. So wird beim Sputterätzen Material an denjenigen Stellen abgetragen, die nicht von Lack geschützt sind.

Nach dem die Probe geätzt ist, wird der Lack von der Oberfläche entfernt. Dafür wird die Probe in einen Behälter mit Isopropanol gebracht und bei $50{ }^{\circ} \mathrm{C}$ etwa 90 Minuten lang im Ultraschallbad bei Stufe 2-3 (niedrige Stärke) geschwenkt. 


\subsubsection{Sputterätzen}

Zum Abtragen der Schichten während des Ätzvorgangs wird die Methode des inversen Sputterätzens verwendet. Dafür werden ähnlich wie beim konventionellen Sputtern die Argon-Atome ionisiert, jedoch anstatt auf den Target werden sie beim Sputter-Ätzen auf die Probe beschleunigt. Dies führt zu der Materialabtragung von der Probenoberfläche. Von den mit Lack bedeckten Flächen wird zunächst der Lack abgetragen und die Schichten der Tunnelelemente werden somit vom Lack von der Abtragung geschützt. Da die Lackschichtdicke die Dicke der Tunnelelemente deutlich überschreitet (Tunnelelemente sind $<50 \mathrm{~nm}$ und Lackschicht ist 1,27 $\mu \mathrm{m}$ dick), können die Flächen bis ins Substrat geätzt werden ohne dass der Lack komplett abgetragen wird. Eine ausführliche Beschreibung des inversen Sputterätzers ISO 90 befindet sich in der Anleitung [45].

Für die Ätzrate haben sich folgende Parameter als essentiell erwiesen: mate-

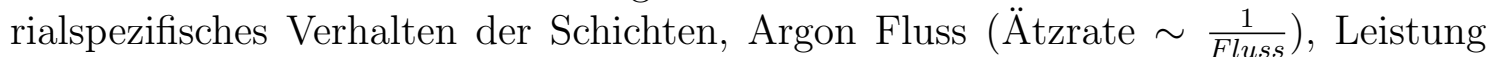
(䒫zrate $\sim$ Leistung) und Zeit (Ätzrate $\sim$ Zeit). Alle diese Parameter bis auf Materialkonstante können auf langen Zeitskalen (ca. 6-12 Monate) variieren und müssen regelmäßig kalibriert werden. Der Argon Fluss kann per Augenmaß so eingestellt werden, dass der Strahl ohne zu divergieren oder sich auf eine kleinere Fläche zu fokussieren von der Quelle laufen muss. Ist das gewährleistet, so kann die verwendete Leistung gewählt werden. Eine langsame Ätzrate bedeutet höhere Genauigkeit bei der Abtragungstiefe, denn die Kontrolle über die geätzte Tiefe erfolgt über die Zeit.

\subsubsection{Strukturierung mehrerer Kontakte}

Mit Hilfe der Elektronenstrahllithographie (engl. sog. e-beam lithography) können Tunnelelementgrößen bis zu $100 \mathrm{~nm}$ reduziert werden. Diese Methode wird in dieser Arbeit nicht ausführlich beschrieben. Eine Beschreibung der e-beam Lithographie befindet sich in der Masterarbeit von J. C. Leutenantsmeyer [46]. Prinzipiell funktioniert die Elektronenstrahllithographie ähnlich wie die optische Lithographie: die Probe wird mit einem elektronenstrahlempfindlichen Lack beschichtet, der während des Argon-Ätzens die Schichten vom Argon-Plasma schützt. Anschließend wird der Lack entfernt. Der Hauptunterschied zur optischen Lithographie liegt darin, dass bei der Belichtung statt UV-Licht ein Elektronenstrahl verwendet wird. Der Vorteil der Elektronenstrahllithographie liegt vor allem an der kleinen Wellenlänge der Elektronen, wodurch deutlich kleinere Elementgrößen erlaubt werden. 

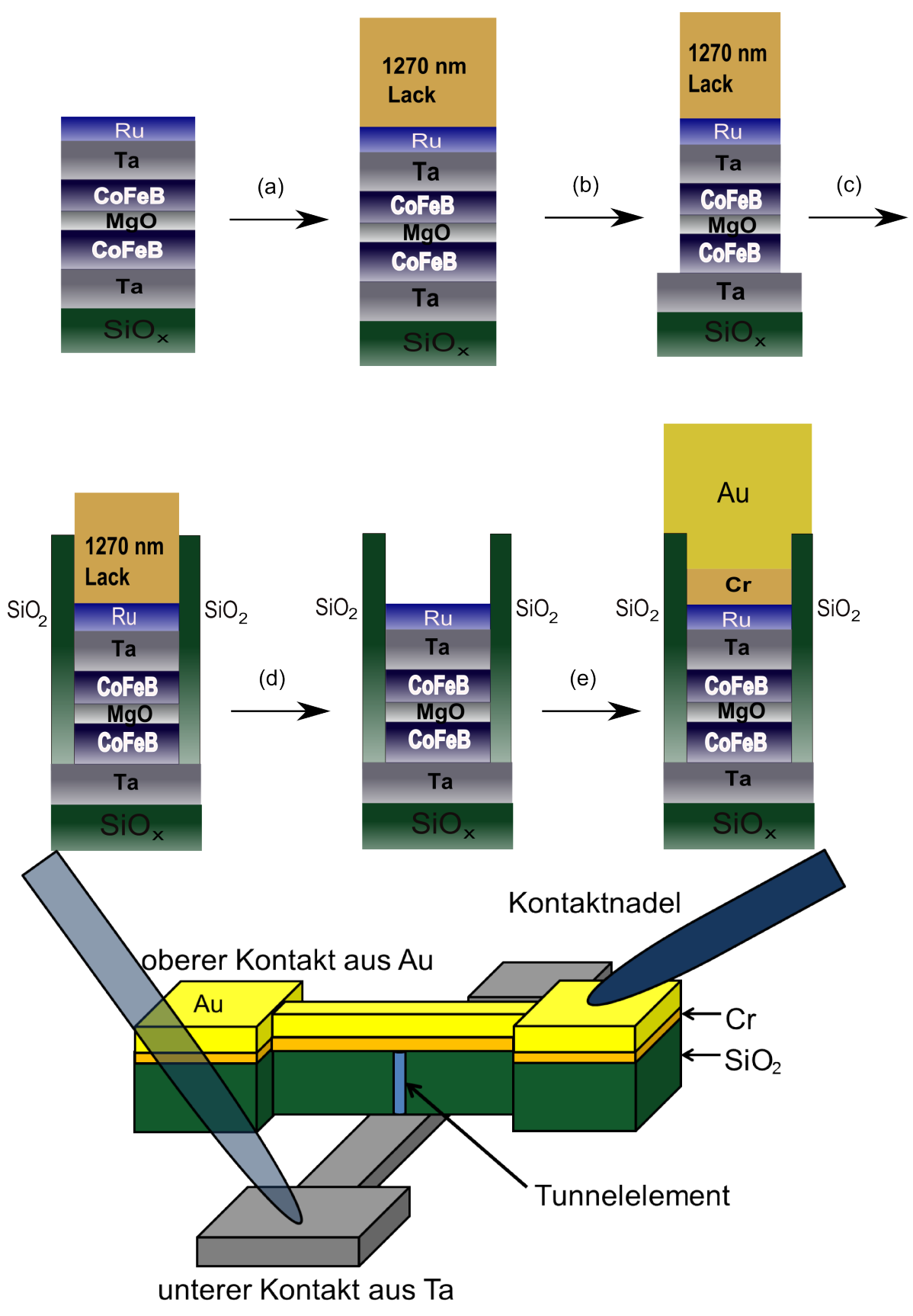

Abbildung 3.2.: Skizze des Lithographie-Prozesses: (a) vollflächiger Schichtstapel wird mit einer Bondpad-Maske belackt und belichtet. Danach bis zur $\mathrm{SiO}_{x}$-Schicht geätzt und der Lack wird entfernt. (b) Die Probe wird belackt und mit einer Tunnelelement-Maske am Bondpad belichtet. Anschließend wird bis zu dem unteren Kontakt geätzt. (c) Isolatorschicht (hier $\mathrm{SiO}_{2}$ ) wird aufgedampft. (d) Bei einem sog. Lift-off Prozess wird der Lack entfernt. (e) Chrom und Gold, die als oberer Kontakt dienen werden, werden aufgedampft. (Unteres Bild) Schritte (a) und (b) werden für den oberen Kontakt wiederholt, so dass am Ende eine BondpadStruktur entsteht. Abbildung nach [18]. 
Die Durchmesser der kleinsten Elemente liegen bei etwa $100 \mathrm{~nm}$. Dies macht die Kontaktierung via Manipulatoren der Probestation unmöglich. Zur Kontaktierung solcher Elemente muss auf die Mehrschrittlithographie zugegriffen werden. Dabei werden einige Mikrometer große Kontakte für die Tunnelelemente hergestellt, die an der Probestation kontaktiert werden können ohne das Element mechanisch zu zerstören.

In Abbildung 3.2 ist der Mehrschrittlithographieprozess als Skizze dargestellt worden. Zunächst kann der Schichtstapel mit der Struktur des unteren Kontakts ${ }^{1}$ belackt, belichtet und geätzt werden (Schritte (a) und (b) der Abbildung 3.2). Nach dem Entfernen vom Lack (Lift-Off Prozess), kann das Element auf dem Kontakt strukturiert werden. Dafür wird der Schritt (a) der Abbildung 3.2 mit der BondpadMaske durchgeführt. Dabei wird je nach Bedarf entweder bis zum $\mathrm{SiO}_{\mathrm{x}}-\mathrm{Substrat}$ oder nur bis zu der unteren Tantal-Schicht (bei Strukturen mit nur einem Bondpad) geätzt. Anschließend wird bei einem Lift-Off-Prozess Lack abgetragen. Im Schritt (b) wird die Probe belackt und mit einer Tunnelelement-Maske auf den Bondpads belichtet und bis zur Ta-Schicht geätzt (nach dem Ätzen ergibt sich für die gekreuzte Bondpads in Abbildung 3.2 (unten) nur der graue untere Kontakt und das blaue Tunnelelement). Beim Schritt (c) wird noch eine Isolator-Schicht um die Elemente herum aufgedampft (in der Regel wird $\mathrm{SiO}_{2}$ als Isolator verwendet). Danach kann der Lack entfernt werden (d) und der obere Kontakt wird vollflächig aufgedampft (e). Anschließend werden die Schritte (a) und (b) mit einer Bondpad-Maske wiederholt, so dass sich am Ende ein Tunnelelement mit der in Abbildung 3.2 (unten) gezeigten Struktur ergibt.

\subsection{Charakterisierung}

\subsubsection{TMR und STT}

Zur elektrischen Charakterisierung der strukturierten Proben bei Raumtemperatur wird die Probestation der Firma Süss verwendet. Der Vorteil der Messungen an der Probestation liegt darin, dass die Kontaktierung der Tunnelelemente direkt mit Kupfer-Beryllium-Nadeln erfolgt. Die Nadeln sind zusätzlich mit Gold beschichtet, wodurch die Kontaktierung aufgrund der niedrigen Goldhärte „weich“ verläuft und die Tunnelelemente geschont werden. Mit Hilfe eines Lichtmikroskops können die Nadeln direkt an die Kontakte mit einer Größe von über $5 \times 5 \mu^{2}$ angebracht werden. Die Kontaktierung wird durch die mit Mikrometerschrauben ausgestatteten Manipulatoren, die in drei Raumrichtungen verstellt werden können, durchgeführt. Wesentlich kleinere Elemente können mit dieser Methode nicht direkt kontaktiert werden. Diese Limitierung wird vor allem durch den Nadelradius von $20 \mu \mathrm{m}$, durch die Genauigkeit der Mikrometerschrauben der Manipulatoren und durch die Vergrößerung des Lichtmikroskops gesetzt. Deutlich kleinere Tunnelelemente können

\footnotetext{
${ }^{1}$ Strukturierung des unteren Kontakts ist oft optional, da die Möglichkeit besteht über die großflächige Umrandung des Maskenabbildes zu kontaktieren.
} 
an der Probestation nur mit einer geeigneten Mehrschritt-Strukturierung mit unteren und oberen Kontaktzuleitungen vermessen werden.

Bei der elektrischen Charakterisierung der magnetischen Tunnelelemente ist oft das Anlegen und Steuern eines äußeren Magnetfeldes während der Messung von großer Bedeutung. Zum diesen Zweck wurden zwei Elektromagnete entwickelt: einer mit in-plane und einer mit out-of-plane Magnetfeldausrichtung. Beide Elektromagneten können Felder bis ca. $\pm 100 \mathrm{mT}$ erzeugen und die Schrittweite kann auf minimal 0,1 mT eingestellt werden.

Nach der Kontaktierung kann variable (bei der Messung der IU-Kennlinien) oder konstante (bei der TMR-Messung) Spannung angelegt werden und die Stromstärke wird vermessen. Dies kann in Abhängigkeit von dem äußeren Magnetfeld mit variablem (TMR-Messung) oder konstantem (STT-Messung) Magnetfeld gemacht werden. Die Steuerung der Variablen und Aufnahme der Messparameter geschieht durch in der Arbeitsgruppe geschriebene LabView Programme ${ }^{2}$.

\subsubsection{Messung des magnetooptischen Kerr-Effekts}
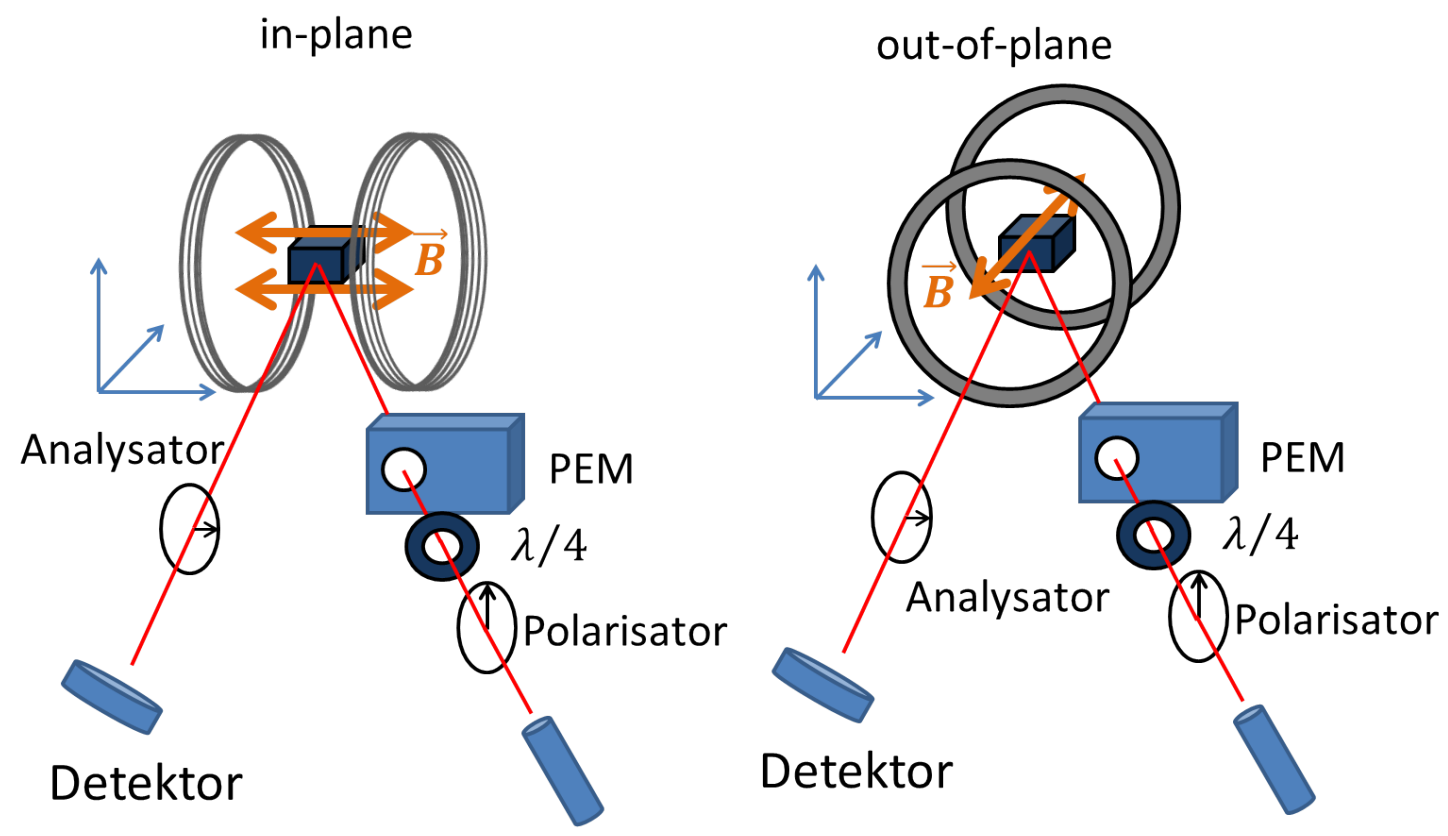

Abbildung 3.3.: Positionen der Heimholzspulen für in-plane und out-of-plane Ausrichtungen des Magnetfeldes und Messaufbau zur Messung der Kerrdrehung.

Zur Untersuchung des magnetischen Verhaltens der ferromagnetischen Schichten eignet sich die Betrachtung derer Hysterese-Kurven. Dafür können die Hysteresen mit Hilfe des magnetooptischen Kerr-Effekts (kurz MOKE) vermessen werden.

\footnotetext{
${ }^{2}$ Geschrieben von Gerrit Eilers und Marvin Walter.
} 
Die zu vermessene Probe wird an einen Probenhalter angebracht und befestigt. Der Probenhalter befindet sich in einem durch die Helmholtzspulen erzeugten Magnetfeld. Das Feld der Spulen kann mit einer Schrittweite von 0,1 mT verfahren werden. Die maximale Feldstärke der Spulen liegt bei etwa $\pm 50 \mathrm{mT}$. Bei den Messungen wurden zwei MOKE-Aufbauten verwendet, die sich nur durch die Position der Helmholtzspulen unterscheiden. Die Spulen des zweiten Aufbaues sind um 90 gedreht, so dass die beiden optische Einrichtungen entsprechend Magnetfelder in die in-plane und out-of-plane Richtung bezüglich der Probe erzeugen können (siehe Abbildung 3.3). Dies ermöglicht Untersuchung des magnetischen Verhaltens der Proben in drei Raumrichtungen. Der Probenhalter wird mittig in dem Magnetfeld der Helmholtzspulen platziert, damit das Magnetfeld homogen im Bereich der Probe verläuft.

Der Aufbau (siehe Abbildung 3.3) funktioniert wie folgt: ein Laserstrahl (ca. $\mathrm{P}=2$ $\mathrm{mW}, \lambda=633 \mathrm{~nm}$ ) wird auf einen Polarisator gelenkt. Der Strahl wird in dem Polarisator linear polarisiert. Beim Durchgang durch das $\lambda / 4$ - Plättchen wird das linear polarisierte Licht zirkular polarisiert. Der Vorteil des $\lambda$ - Kompensators ${ }^{3}$ ist, dass damit einige störende Effekte (z. B. Elliptizitäten) kompensiert werden können. Der photoelastischer Modulator (kurz PEM) verschiebt die Phase um $\pm \frac{\pi}{2}$ mit einer Frequenz $f=50 \mathrm{kHz}$. Das auf die Probe einfallende Licht wird reflektiert und passiert dabei den Analysator, an dem der horizontaler Anteil des Signals abgetrennt wird (siehe Abbildung 3.3). Dies hat zur Folge, dass Signal und Modulation gleiche Frequenz aufweisen und somit verglichen werden können [47].

\subsubsection{Zeitaufgelöster magnetooptischer Kerr-Effekt}

Eine weitere Charakterisierungsmethode ist die Messung des zeitaufgelösten magnetooptischen Kerr-Effekts (engl. time-resolved magneto optical Kerr effekt, kurz TRMOKE). Der Aufbau ist in Abbildung $3.4 \mathrm{zu}$ sehen. Der Aufbau ähnelt der MOKE-Messungen. Für die Messung wird ein Titan-Saphire-Laser benutzt, der Laser-Pulse im Femtosekundenbereich aussenden kann. Die Breite der Laser-Pulse und somit auch die zeitliche Auflösung liegt in der Regel zwischen 40-60 fs. Der Hauptunterschied zum im Unterabschnitt 3.3.2 beschriebenen MOKE-Aufbau liegt in der Verwendung eines Pumpstrahls, der die Probe erhitzen kann. Die Zeitdifferenz zwischen den Pulsen des Pumpstrahls liegt bei $4 \mu \mathrm{s}$. So wird das System in zwei Zustände versetzt: Pumpstrahl trifft die Probe und Pumpstrahl trifft die Probe nicht. Während der Strahl die Probe nicht erhitzt, kann die Probe abkühlen, so dass eine weitere Messung unter ähnlichen Bedingungen durchgeführt werden kann. Die

\footnotetext{
${ }^{3}$ Das verwenden des $\lambda / 4$ - Plättchen ist bei dem Aufbau optional. Bei der in-plane Anordnung wurden Aufnahmen mit und ohne $\lambda / 4$ - Kompensator für unterschiedliche Lock-In-Einstellungen durchgeführt und verglichen. Die Einstellung, bei der die Messung ohne $\lambda / 4$ - Plättchen gleiche Ergebnisse wie mit der Komponente lieferte (2f - Einstellung am Lock-In, die der Messung der Kerr-Drehung entspricht), wurde notiert und für spätere Messungen übernommen. Bei den Messungen in die out-of-plane Richtung wurde der $\lambda / 4$-Kompensator stets zur Verbesserung des Signal-Rausch-Verhältnisses verwendet.
} 
Energiedichte des Pumpstrahls kann durch eine Blende variiert werden und liegt in der Regel zwischen $10 \frac{\mathrm{mJ}}{\mathrm{cm}^{2}}$ und $50 \frac{\mathrm{mJ}}{\mathrm{cm}^{2}}$. Die zentrale Wellenlänge des Pulses liegt bei 790-800 nm. Die Durchmesser des Pump- und Messstrahls liegen bei entsprechend $\sim 60 \mu \mathrm{m}$ und $\sim 30 \mu \mathrm{m}$ [17]. Für die Doppel-Modulation wird ein Chopper mit einer Frequenz von $800 \mathrm{~Hz}$ und PEM mit einer Frequenz von $50 \mathrm{kHz}$ verwendet.

Bei dem Aufbau lässt sich ebenfalls die Elektromagnetspule austauschen, so dass ein Wechsel zwischen den äußeren in- und out-of-plane Magnetfeldern vorgenommen werden kann. Der optische Strahlengang wird bei Umstellung nicht betroffen und entspricht der Abbildung 3.4.

Zusätzlich zu dem in Abbildung 3.4 angezeichnetem Aufbau wird für den Pumpstrahl noch eine Delay-Stage benutzt, die für die Erhöhung der Weglänge des Pumpstrahls sorgt und somit eine kontrollierte Verschiebung des Zeitpunktes, an dem der Pumpstrahl die Probe trifft, erzeugt. Die maximale zeitliche Verzögerung, die durch die Delay-Stage hervorgerufen werden kann, liegt bei etwa 1 ns. Vom Prinzip her sorgt die Delay-Stage nur für eine Erhöhung der optischen Weglänge des Pumpstrahls durch eine Verschiebung des Spiegels, der noch vor der Probe eingebaut ist.

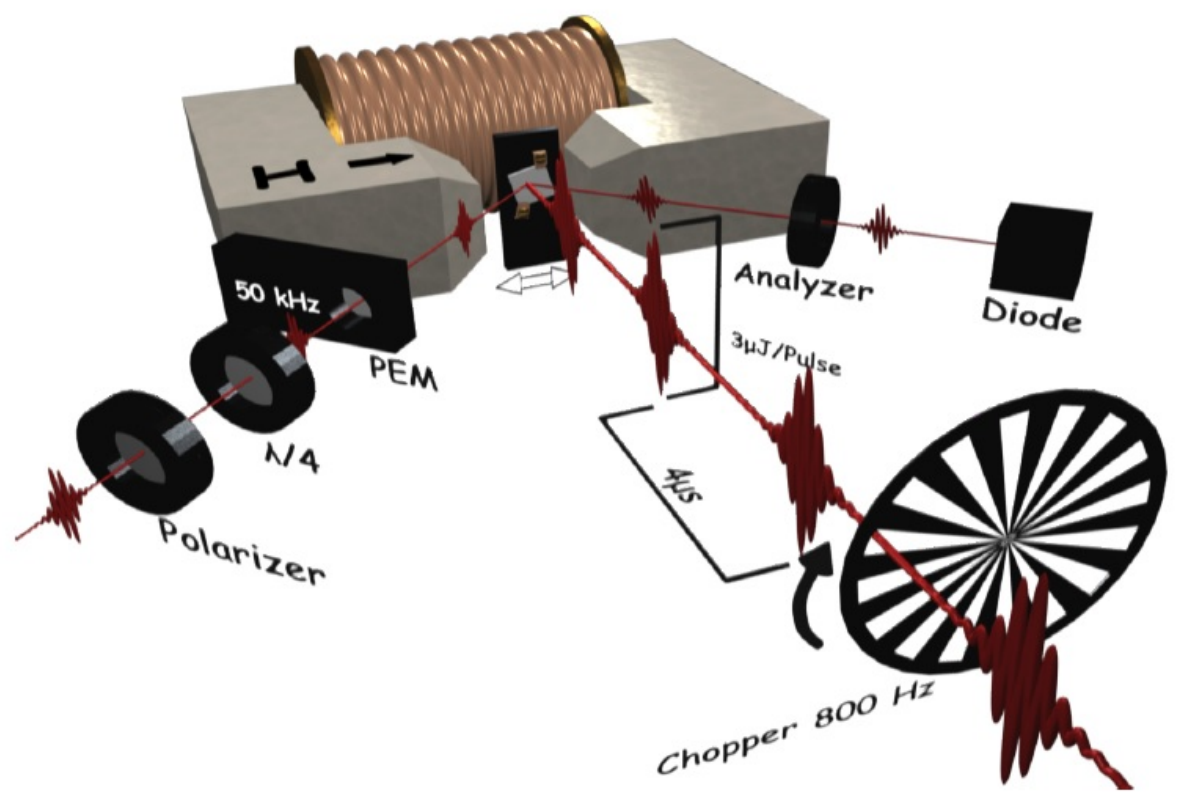

Abbildung 3.4.: Der TRMOKE-Aufbau unterscheidet sich von der MOKEMessmethode vor allem durch den Pulslaser. Der Laserstrahl wird von einem Strahlspalter in zwei Strahlen gespalten. Der eine Strahl wird Probe-Strahl genannt und durchläuft auf dem Strahlengang die gleichen optischen Geräte wie der Strahl aus dem MOKE-Aufbau. Der zweite Strahl (genannt Pumpstrahl) wird zur Energieübertragung auf die Probe genutzt. Der Pumpstrahl durchläuft einen Chopper mit einer Frequenz von $800 \mathrm{~Hz}$. Das zeitliche Intervall zwischen den Pump-Pulsen beträgt 3 us. Aus [17]. 



\section{Experimentelle Ergebnisse}

In diesem Kapitel werden experimentelle Ergebnisse präsentiert und diskutiert. Das Kapitel ist wie folgt gegliedert: In dem ersten Abschnitt 4.1 werden die während dieser Arbeit durchgeführten Untersuchungen der magnetischen Anisotropie der hier verwendeten CoFeB-Legierung gezeigt (siehe Kapitel "Experimentelle Methoden"). Das Verhalten der Anisotropie in Abhängigkeit von dem Schichtsystem und dessen Parametern wird diskutiert. Im Abschnitt 4.2 werden die Spin-TransferTorque-Messungen für Proben mit senkrechter magnetischer Anisotropie präsentiert. Die charakteristischen Schaltströme werden ermittelt und das Verhalten der IUKennlinien sowie die Eigenschaften der Tunnelelemente werden diskutiert. Der Abschnitt 4.3 wird sich mit dem Verhalten der Magnetisierung auf ultrakurzen Zeitskalen beschäftigen.

Während dieser Arbeit entstanden viele interessante und effiziente Kollaborationen zwischen mehreren Arbeitsgruppen. Im Folgenden wird ein kurzer Überblick über die Arbeitsaufteilung und meinem eigenen Beitrag zu dieser Arbeit gegeben.

Von mir wurden folgende Experimente und Auswertungsschritte gemacht:

- Vorarbeit zur Herstellung, Optimierung und erste Charakterisierung der Proben mit senkrechter Anisotropie. Dafür wurden mehrere Probenreihen gemacht, an denen anschließend der magnetooptischer Kerr-Effekt vermessen wurde.

- Auswertung dieser Daten, Optimierung der Proben.

- Entwicklung der Schritte für die Probenstrukturierung (mit optischer Lithographie) und Argonätzen.

- Elektrische Charakterisierung der Proben.

- Probenherstellung, Untersuchung und Analyse der Proben aus dem Abschnitt 4.1.

- Analyse und Interpretation der Daten aus dem Abschnitt 4.2.

- Vorarbeit zur Gestaltung des Schichtenstapels aus dem Abschnitt 4.3. Vermessung der Proben, Analyse und Interpretation der Daten.

Folgende Schritte wurden von Kollegen gemacht: 
- Die Ausarbeitung der Schritte für die Probenstrukturierung mit Hilfe der Elektronenstrahllithographie (Johannes Christian Leutenantsmeyer).

- HRTEM-Aufnahmen und EELS-Untersuchung der Proben (Henning Schuhmann und Patrick Peretzki, IV. Physikalisches Institut, Georg-August-Universität Göttingen).

- Probenherstellung für die Ultrakurzzeitmessungen (Ulrike Martens, Physikalisches Institut, Universität Greifswald).

- Diskussion und Vorschläge zur Entwicklung des Schichtenstapels mit senkrechter Anisotropie (Rashid Gareev, Universität Regensburg).

\subsection{Magnetische Anisotropie der CoFeB/MgO-basierten Tunnelelemente.}

\subsubsection{Vorwort}

Magnetische Tunnelelemente mit senkrechter Anisotropie sind für aktuelle Forschung und Anwendungen von großem Interesse. Besonders attraktiv für die möglichen Anwendungen werden magnetisch senkrecht ausgerichtete Elemente ${ }^{1}$ durch die im Vergleich zur in-plane Tunnelelementen deutlich niedrigere Schaltströme. Solche theoretisch vorhergesagte Eigenschaften wurden bereits experimentell nachgewiesen [27]. Es besteht jedoch immer noch eine Reihe von offenen Fragen und Optimierungsmöglichkeiten. Dieses Kapitel beschäftigt sich mit der Untersuchung der für die PMA verantwortlichen Parameter.

\subsubsection{Abhängigkeit der Magnetischen Anisotropie von der CoFeB-Schichtdicke.}

Zur Untersuchung des magnetischen Verhaltens der CoFeB-Schicht, wurde eine Probe mit einem Schichtdickengradienten (siehe Unterunterabschnitt 3.1.2.1) hergestellt und die Kerrdrehung vermessen. Der Schichtstapel der Probe ist in Abbildung 4.1 (c) skizziert. Durch den Schichtdickengradienten ist die Dicke der CoFeB-Schicht der Probe variabel und liegt zwischen 1,2 und 1,6 nm. Nach der Herstellung wurde die Probe für eine Stunde bei $300{ }^{\circ} \mathrm{C}$ in der Annealing-Kammer ausgelagert (siehe Unterabschnitt 3.1.3). An dieser Probe wurde anschließend MOKE vermessen. Dabei wurden mehrere Punkte mit gleicher Schrittbreite entlang des Gradienten vermessen. Die Messung wurde für die in- und out-of-plane Feldrichtungen durchgeführt.

\footnotetext{
${ }^{1}$ Es werden in der Literatur zur Beschreibung dieser Effekte englische Begriffe bevorzugt. Dem "magnetisches Tunnelelement mit senkrechter magnetischer Anisotropie" entspricht das englische "magnetic tunnel junction with perpendicular magnetic anisotropy". Abgekürzt: MTJ with PMA. Im Folgenden Text wird diese Abkürzung verwendet.
} 
(a)

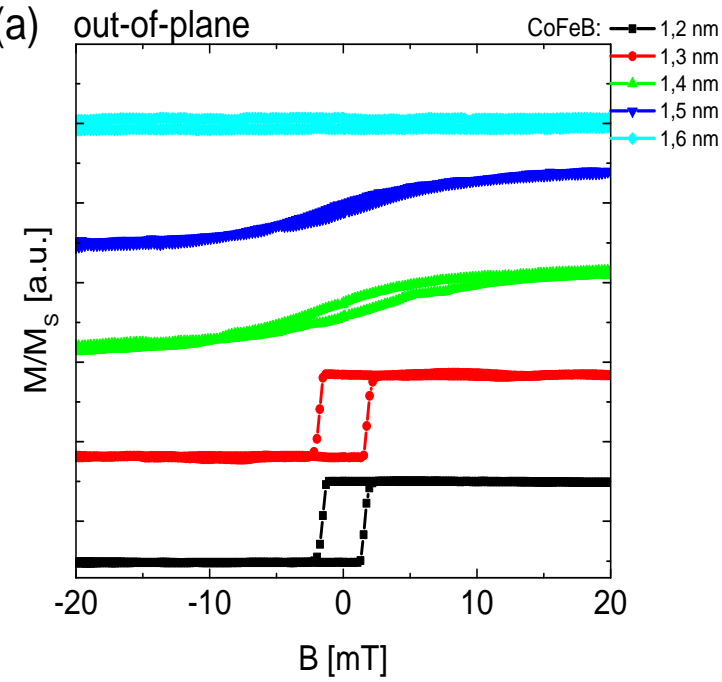

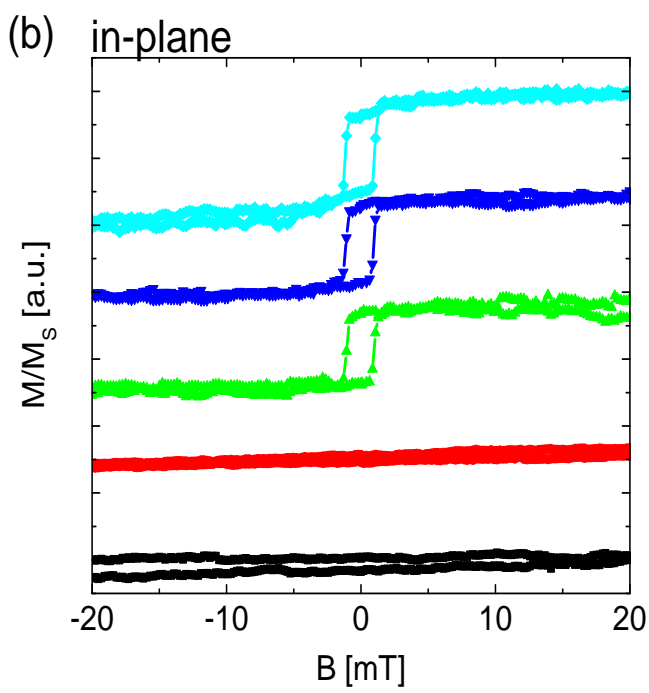

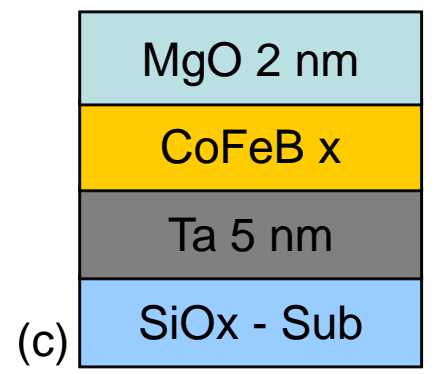

Abbildung 4.1.: MOKE-Messungen in die out-of-plane (a) und in-plane (b) Richtungen. (c) Skizze des Schichtenstapels.

In Abbildung 4.1 (a) und (b) sind die Ergebnisse der MOKE-Messungen präsentiert. Die jeweilige Grafiken repräsentieren unterschiedliche Schichtdicken der CoFeB-Schicht. Die Kurven wurden für eine bessere Übersicht gegeneinander verschoben. Die Messungen zeigen einen klaren Trend: Mit steigender CoFeB-Schichtdicke ändert sich auch das magnetische Verhalten und zwar wenn für die CoFeBSchichtdicke von 1,2-1,3 nm die leichte Achse in die out-of-plane Richtung liegt, so ändert sich die Richtung der leichten Achse ab etwa 1,5 nm CoFeB in die in-plane Richtung.

Damit der Trend klarer wird, wurden die Sättigungsfelder aus den Graphen ermittelt (siehe Tabelle 4.1) und in Abbildung 4.2 gegen die Schichtdicke aufgetragen. Der Bereich, der die kritische CoFeB-Dicke enthält, ist rot-schraffiert markiert. In diesem Bereich findet der Übergang von out-of-plane zur in-plane Anisotropie statt. Die kritische CoFeB-Dicke liegt somit bei 1,35 \pm 0, $025 \mathrm{~nm}$.

Zur weiteren Untersuchung der magnetischen Eigenschaften werden die entsprechenden Anisotropie-Werte berechnet. Dies geschieht nach dem Stoner-WohlfahrthModell. Um die Anisotropie-Werte berechnen zu können, muss zunächst der hysteretische Beitrag von den jeweiligen Hysteresen abgezogen werden. Idealisiert sollten 


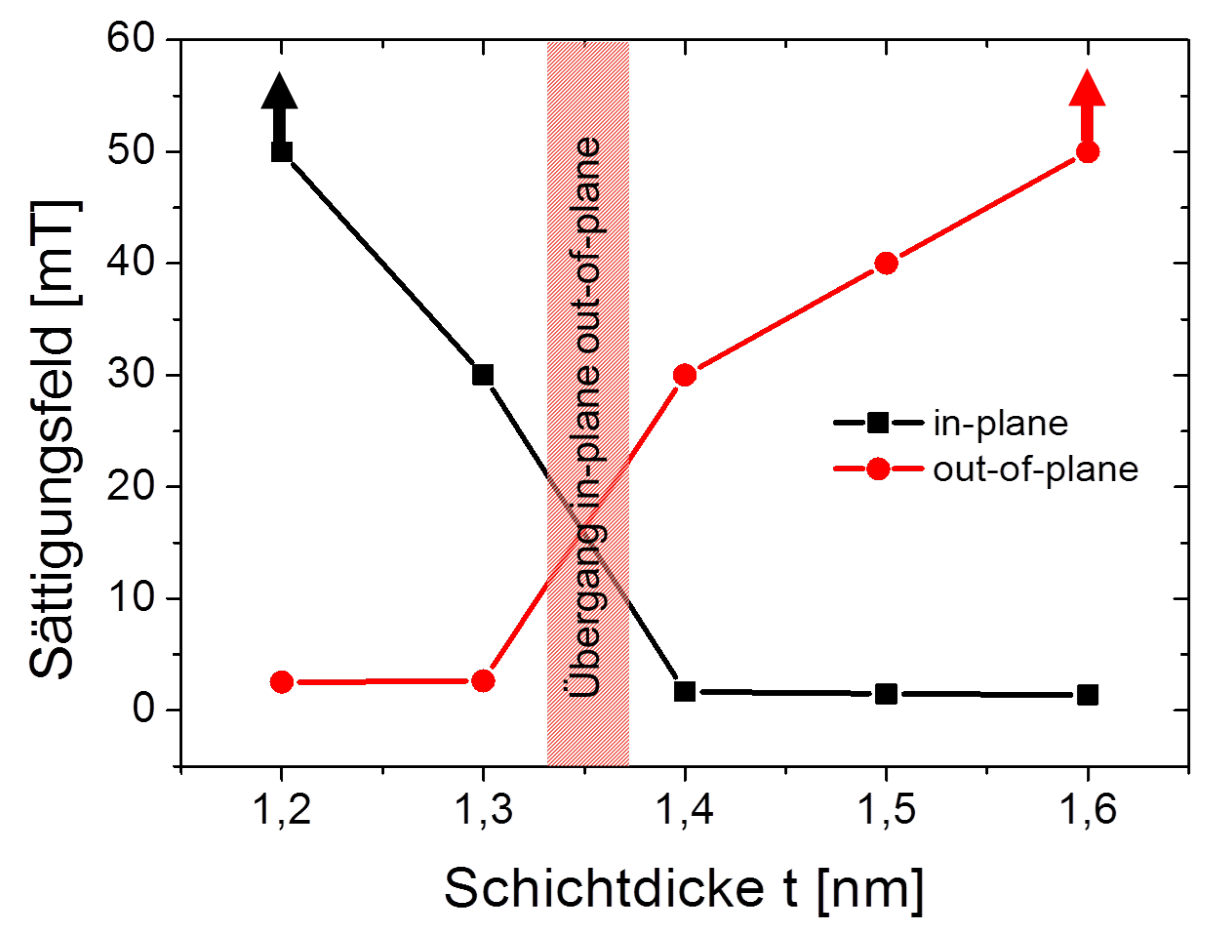

Abbildung 4.2.: Sättigungsfelder in Abhängigkeit von der Schichtdicke. In-plane Richtung - schwarze Punkte, out-of-plane - rote Punkte. Die mit den Pfeilen markierte Punkte wurden auf $50 \mathrm{mT}$ abgerundet, da keine Sättigung bei der entsprechenden Feldausrichtung für diese CoFeB-Schichtdicken gemessen werden konnte.

\begin{tabular}{|c|c|c|}
\hline $\begin{array}{c}\text { Schichtdicke } \\
{[\mathrm{nm}]}\end{array}$ & $\begin{array}{c}\text { Sättigungsfeld } \\
{[\mathrm{mT}]}\end{array}$ & $\begin{array}{c}\text { Sättigungsfeld } \\
{[\mathrm{mT}]}\end{array}$ \\
\hline & in-plane & out-of-plane \\
\hline \hline 1,2 & 50 & 2,53 \\
\hline 1,3 & 30 & 2,65 \\
\hline 1,4 & 1,7 & 30 \\
\hline 1,5 & 1,45 & 40 \\
\hline 1,6 & 1,4 & 50 \\
\hline
\end{tabular}

Tabelle 4.1.: Sättigungsfelder abhängig von der Schichtdicke. 


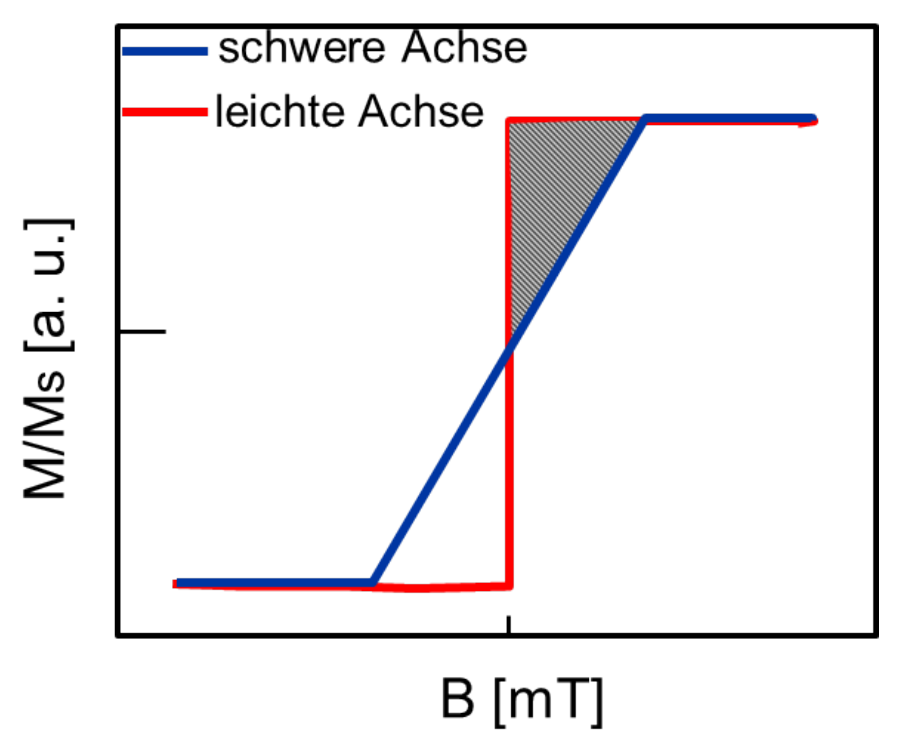

Abbildung 4.3.: Veranschaulichung der Anisotropieberechnung aus dem StonerWohlfahrt-Modell.

die Kurven danach wie in Abbildung 4.3 aussehen. Berechnet man die schraffierte Fläche (Differenz zwischen den Integralen der beiden Kurven), so entspricht sie der Energie, die benötigt wird, um die Magnetisierung von der leichten zur schweren Achse zu drehen. Diese Energie entspricht per Definition der magnetischen Anisotropie (siehe Abschnitt 2.3).

Die berechneten Anisotropien sind in Abbildung 4.4 zu sehen. Der Punkt für $1 \mathrm{~nm}$ CoFeB wurde mit SQUID ${ }^{2}$ vermessen (siehe Abbildung 4.5). Die restlichen Punkte stammen aus MOKE-Messungen. Die Berechnung der Anisotropie erfolgte folgendermaßen: Zunächst wurden die Energien, die benötigt werden, um die Schicht in jeweilige Richtung zu sättigen, berechnet. Dafür wurde über den nicht hysteretischen Teil der Hysteresen bis zum Sättigungspunkt integriert. Anschließend wurden die beiden Energien (für leichte und schwere Achsen) voneinander subtrahiert, so dass das Ergebnis der magnetischen Anisotropie entsprach. Analog wurde die Rechnung für alle Messungen durchgeführt, bei denen das Sättigungsfeld für beide magnetische Achsen erreicht wurde. Messungen bei denen es nicht der Fall war wurden für diesen Schritt nicht verwendet.

Für die effektive Anisotropie gilt folgende Formel [25]:

$$
K_{\mathrm{eff}} \cdot t=K_{b} \cdot t+K_{i}
$$

$K_{b}$ entspricht der Differenz zwischen dem Volumenbeitrag und der Formaniso-

\footnotetext{
${ }^{2}$ Dafür wurde eine weitere Probe hergestellt: $\mathrm{SiOx} / \mathrm{Ta} 15 \mathrm{~nm} / \mathrm{CoFeB} \mathrm{X} / \mathrm{MgO}$ und für X=1 nm am SQUID gemessen.
} 


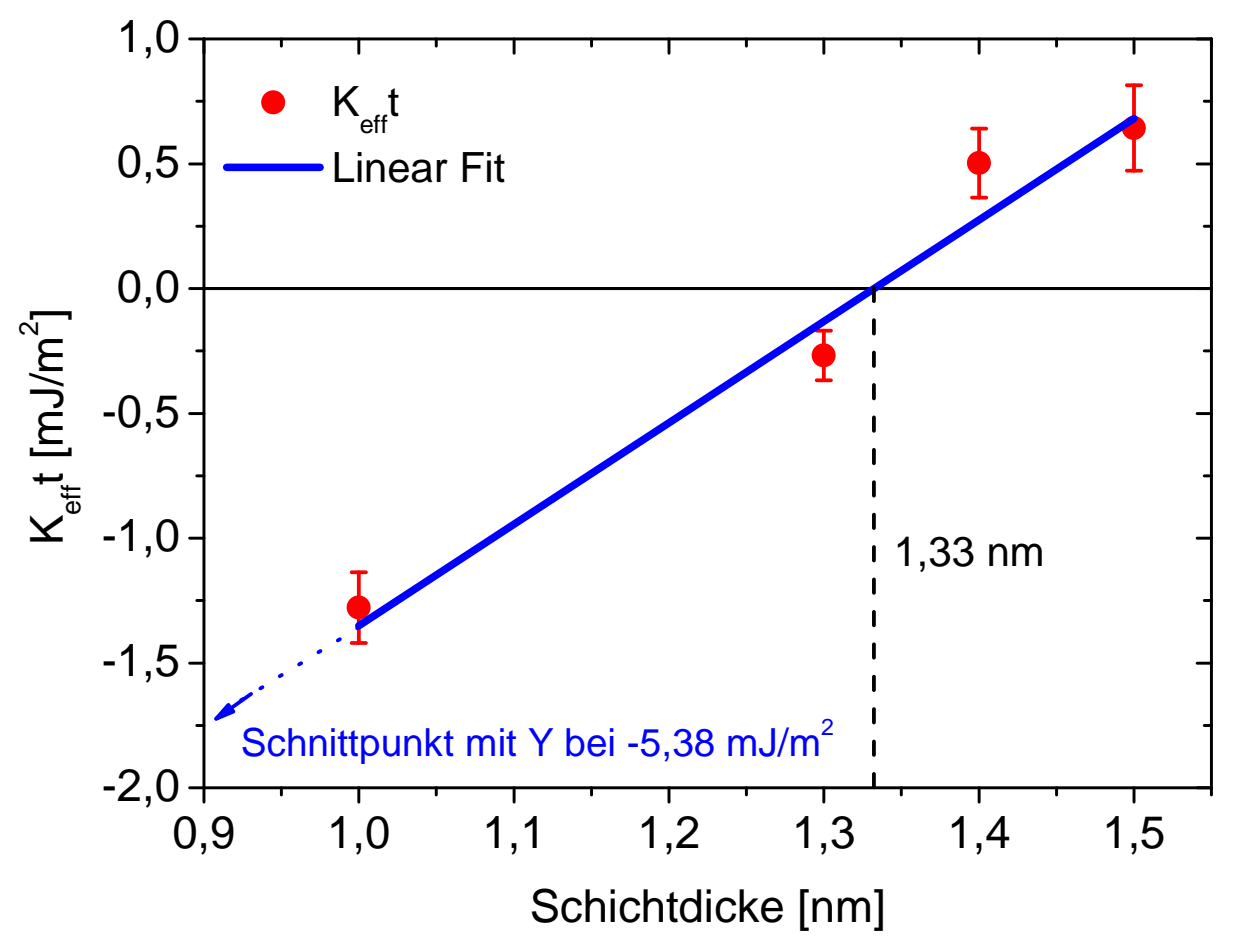

Abbildung 4.4.: Anisotropieverhalten in Abhängigkeit von der CoFeBSchichtdicke. 1,33 nm CoFeB entspricht der kritischen Schichtdicke, bei der die Anisotropie in beide Richtungen zeigen kann.

tropie $K_{b}=K_{V}-\frac{M_{S}^{2}}{2 \mu_{0}}[27,26], K_{i}$ ist die Grenzflächenanisotropie und $t$ - CoFeBSchichtdicke.

Trägt man $K_{\text {eff }} \cdot t$ gegen $t$ ein (siehe Abbildung 4.4), so kann man durch Anlegen einer Geraden (Gleichung 4.1) die Werte für $K_{b}=404 \pm 62\left[\frac{\mathrm{mJ}}{\mathrm{cm}}\right]$ und $K_{i}=(5,38 \pm$ $0,81) \cdot 10^{-4}\left[\frac{\mathrm{mJ}}{\mathrm{cm}^{2}}\right]$ berechnen. Für sehr dünne Schichten des hier verwendeten CoFeB gilt $^{3} M_{S}=1,3 \mathrm{~T}$. Daraus lässt sich der Wert für den Volumenbeitrag der Anisotropie berechnen: $K_{V}=268\left[\frac{\mathrm{mJ}}{\mathrm{cm}^{3}}\right]$. Diese Werte sind vergleichbar mit den Ergebnissen von [25] und [27]. Der Vergleich der Werte ist in der Tabelle 4.2 aufgelistet.

Der Wert für $K_{i}$ liegt in der gleichen Größenordnung, ist jedoch höher als von Lee et al. und Ikeda et al. berichtet wurde. Die kritische Schichtdicke der CoFeB-Schicht liegt bei unseren Proben unter dem Literaturwert. Unsere Proben unterscheiden sich nur im geringen Maß von denen aus der Literatur, jedoch sollen diese Unterschiede nicht unerwähnt bleiben:

- Die beiden Gruppen benutzen $\mathrm{Co}_{20} \mathrm{Fe}_{60} \mathrm{~B}_{20}$ während für diese Arbeit benutzte

${ }^{3}$ Dieser Wert wurde aus den in unserer Arbeitsgruppe hergestellten Schichten mit Hilfe der SQUID-Messungen bestimmt. Der Wert von $M_{S}=1,3 \mathrm{~T}$ entspricht dem von H. Sato et al. [48] berichteten Wert. 


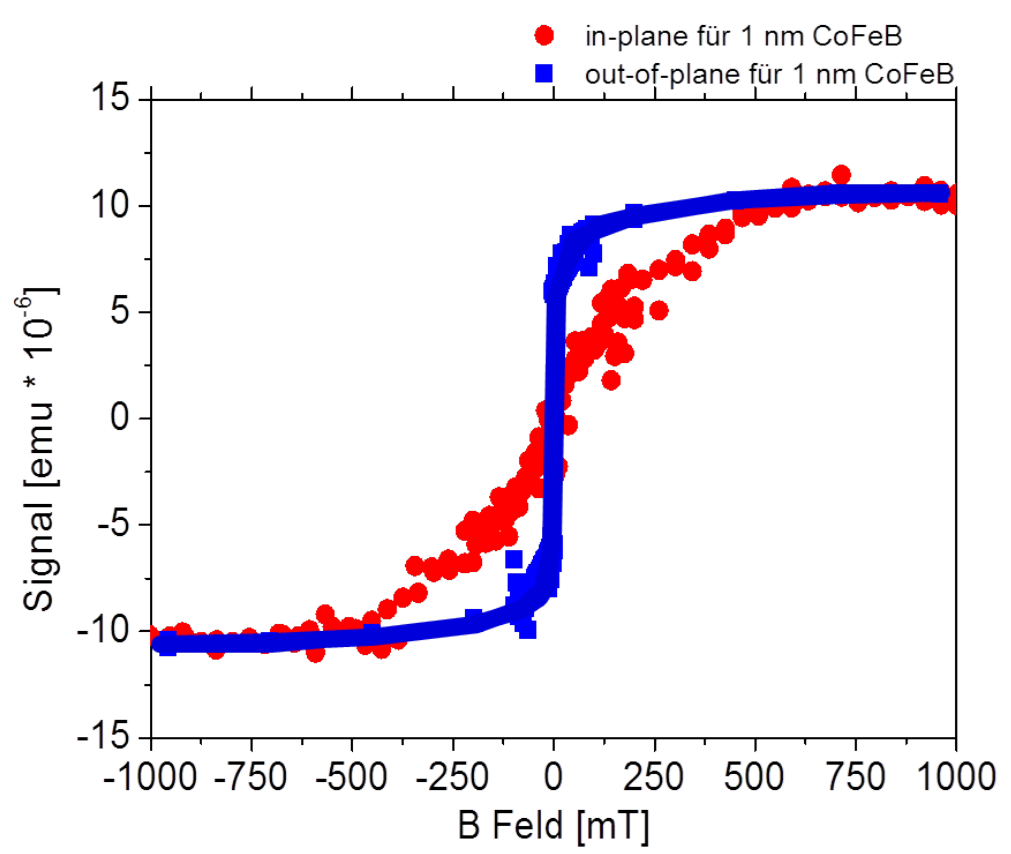

Abbildung 4.5.: SQUID-Messung für $1 \mathrm{~nm}$ CoFeB mit abgezogenem Beitrag des Substrates. Die blauen Punkte entsprechen der Messung in out-of-plane Richtung und die roten Punkte entsprechen der Messung in in-plane Richtung. Die leichte Achse liegt senkrecht zur Probenoberfläche.

Sputter-Target folgende Legierung hat $\mathrm{Co}_{25} \mathrm{Fe}_{55} \mathrm{~B}_{20}$.

- Bei der Auslagerung der hier benutzten Proben wurde kein externes Magnetfeld angelegt.

Insgesamt zeigen unsere Proben ein für solche Schichtsysteme vorhergesagtes Verhalten $[27,26,25,49]$ : die Anisotropie ändert ihr Vorzeichen mit steigender CoFeBSchichtdicke von $K_{\text {eff }}<0$ zu $K_{\text {eff }}>0$ nach dem Erreichen der kritischen Schichtdicke, bei der $K_{\text {eff }}=0$ ist. Diese drei Anisotropie-Zustände entsprechen drei unterschiedlichen magnetischen Zuständen des Gesamtsystems:

- $K_{\text {eff }}<0$ : in diesem Fall ist der $K_{b}$-Beitrag kleiner als der Grenzflächenbeitrag

\begin{tabular}{|c|c|c|c|c|}
\hline & $K_{V}\left[\frac{\mathrm{mJ}}{\mathrm{cm}^{3}}\right]$ & $K_{i} \cdot 10^{-4}\left[\frac{\mathrm{mJ}}{\mathrm{cm}^{2}}\right]$ & Krit. CoFeB-Dicke & Ext. B-Feld \\
\hline \hline Berechnete Werte & $268 \pm 62$ & $5,38 \pm 0,81$ & $1,35 \pm 0,2 \mathrm{~nm}$ & nein \\
\hline Ikeda et al [27]. & 210 & 1,3 & $1,5 \mathrm{~nm}$ & $450 \mathrm{mT}$ \\
\hline Lee at al [25]. & 717 & 1,33 & $>1,5 \mathrm{~nm}$ & $1,5 \mathrm{~T}$ \\
\hline
\end{tabular}

Tabelle 4.2.: Vergleich der Ergebnisse für Volumen- und Grenzflächenanisotropie (entsprechend $K_{V}$ und $K_{i}$ ) mit Literaturwerten. 
$K_{i}$. Da die Grenzflächenanisotropie der PMA entspricht, hat das System in der out-of-plane Richtung die leichte Achse.

- $K_{\text {eff }}=0$ : wenn die kritische Schichtdicke erreicht ist, sind $K_{b}$ und $K_{i}$ gleich. Beide Domänetypen (in- und out-of-plane) haben gleiche Energie. Ist kein externes Magnetfeld angelegt, so fällt das System in den Grundzustand, welches durch Streifendomäne repräsentiert wird.

- $K_{\text {eff }}>0$ : in diesem Fall ist der gesamte Beitrag der Formanisotropie und der Volumenanisotropie $K_{b}$ höher als der Grenzflächenbeitrag $K_{i}$. Da die CoFeBSchicht vollflächig auf die Probe aufgedampft wird und nach dem Aufdampfen eine große Fläche mit sehr kleinen Dicke ist, wird die in-plane Ausrichtung der magnetischen Domänen energetisch günstiger. Dies führt zur einer out-of-plane Anisotropie.

\subsubsection{Einfluss der Diffusionsvorgänge auf die magnetischen Eigenschaften der magnetischen Tunnelelemente}

In diesem Abschnitt wird der Einfluss einer Pufferschicht auf das magnetische Verhalten der MTJ untersucht. Dafür werden zwei Proben betrachtet: Probe $A$ und $B$ (siehe Skizze Abbildung 4.6).

- Probe A: SiOx-Substrat / Ta $5 \mathrm{~nm} / \mathrm{CoFeB}$ x nm / MgO 2 nm.

- Probe B: MgO-Substrat / MgO $5 \mathrm{~nm} / \mathrm{CoFeB}$ x nm / MgO 2 nm.

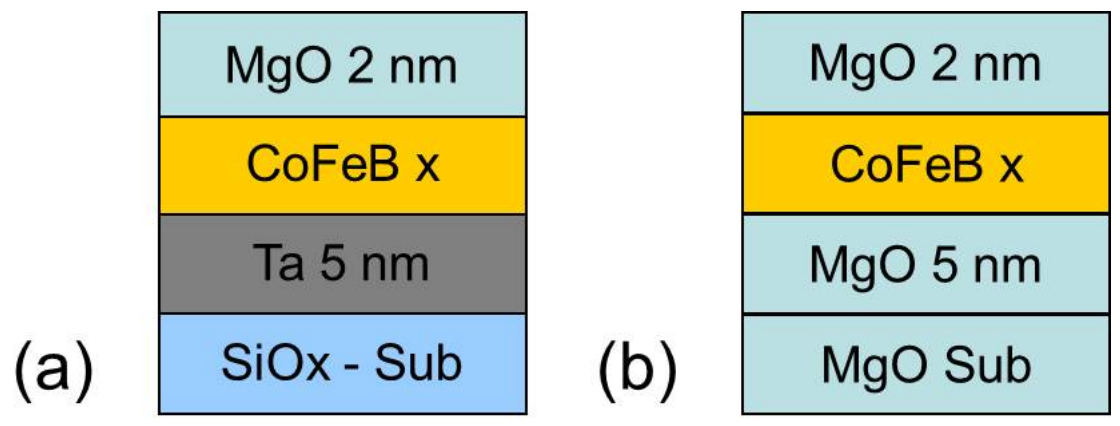

Abbildung 4.6.: Skizzen der Probe A (a) und Probe B (b)

Beide Proben wurden bei $300{ }^{\circ} \mathrm{C}$ eine Stunde lang ohne externes Magnetfeld ausgelagert. An beiden Proben wurde anschließend in der in- und out-of-plane Richtung MOKE gemessen. In Abbildung 4.7 (a) und (b) sind Ergebnisse für die CoFeBSchichtdicke von 1,3 nm abgebildet ${ }^{4}$. Werden die Ergebnisse betrachtet, so stellt

\footnotetext{
${ }^{4}$ Auf die Darstellung der Messungen für weitere CoFeB-Schichtdicken wird auf dieser Stelle verzichtet. Die Messergebnisse werden jedoch im Anhang präsentiert.
} 

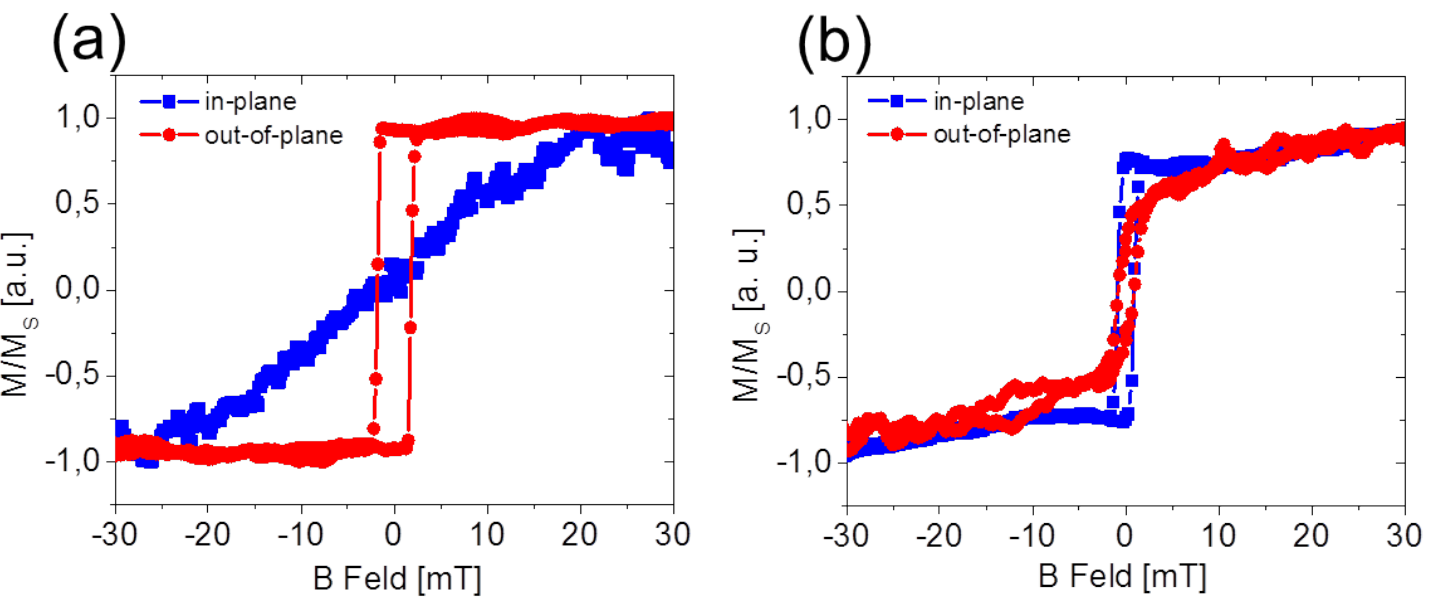

Abbildung 4.7.: Vergleich der Hysteresen für 1,3 nm CoFeB für (a) Probe A mit Tantal als Pufferschicht, (b) Probe B mit MgO als Pufferschicht.

man fest, dass Probe A das typische Verhalten der leichten Achse für die out-ofplane Richtung zeigt. Die in-plane Richtung entspricht dem Verhalten der harten Achse. Bei der Betrachtung der Hysterese-Kurven der Probe B scheint die in-plane Richtung der leichten Achse zu entsprechen. Die out-of-plane Magnetisierung zeigt jedoch nicht das typische Verhalten der harten Achse. Dies lässt vermuten, dass bei der Probe B für 1,3 nm eine Koexistenz der Domänen mit unterschiedlicher Anisotropie möglich ist. Es ist auch möglich, dass die magnetische Anisotropie nicht so stark ausgeprägt ist wie die der Probe A.

Zur weiteren Untersuchung des magnetischen Verhaltens der Probe B wurde analog zur Probe A (siehe Unterabschnitt 4.1.2) die Sättigung für in- und out-of-plane Ausrichtung des Magnetfeldes gegen die Schichtdicke der CoFeB-Schicht aufgetragen. Das Ergebnis ist in Abbildung 4.8 (b) zu sehen. Das Verhalten der Sättigung zeigt einen deutlichen Trend zur in-plane Anisotropie. Vergleicht man Proben A und B miteinander, so stellt man fest, dass Probe B keinen Wechsel der magnetischen Anisotropie mit steigender Schichtdicke der ferromagnetischen Schicht aufweist.

Die beiden Proben A und B unterscheiden sich nur durch die Pufferschicht. Dies bedeutet zwangsläufig, dass die Auswahl der Pufferschicht essentiell für die PMA ist. Der Einfluss der Deck- und Unterschichten auf die MTJs wurde bereits im Bezug auf das TMR-Verhalten in meiner Diplomarbeit diskutiert [18]. Es wurde gezeigt, dass Tantal als Pufferschicht das Kristallisationsverhalten der CoFeB-Schicht nicht daran hindert, während des Auslagerungsschrittes von der MgO-Grenzfläche aus zu kristallisieren. So entsteht ein CoFeB-Kristall, der an die Struktur der MgO-Schicht angepasst ist [18]. Die in Abbildung 4.8 abgebildete Ergebnisse zeigen, dass Tantal auch weitere Einflüsse auf die CoFeB-Schicht haben muss, denn aus dem Blickwinkel der CoFeB-Kristallisation zeigt die Probe B theoretisch bessere Anfangsbedingungen als Probe A: bei der Probe B ist die CoFeB-Schicht zwischen zwei MgO-Schichten platziert und sollte während der Auslagerung von den beiden MgO-Schichten durch- 
(a)

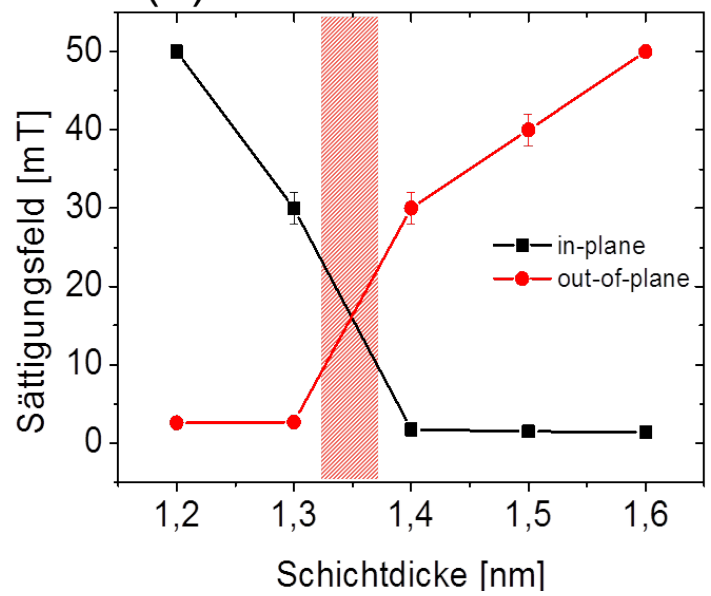

(b)

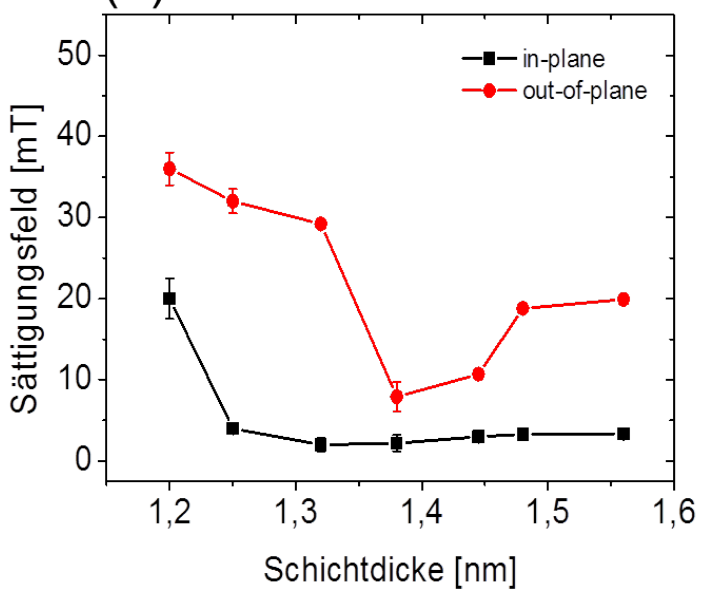

Abbildung 4.8.: Sättigungsfelder: (a) Probe A mit einer Ta-Pufferschicht und (b) Probe B mit einer MgO-Pufferschicht. Bei der Probe B kommt bleibt die leichte Achse in-plane ausgerichtet.

kristallisieren. Einen weiteren Vorteil für PMA sollte die Probe B gegenüber der Probe A durch die zwei $\mathrm{CoFeB} / \mathrm{MgO}-$ Grenzschichten haben, denn der Ursprung der PMA soll an dieser Grenzschicht in Form einer Hybridisierung der Fe 3d und O 2p Orbitalen entstehen [26]. Die Verdopplung der Fläche der $\mathrm{CoFeB} / \mathrm{MgO}-G r e n z s c h i c h t$ sollte zu einer Erhöhung der PMA bei der Probe B im Vergleich zur Probe A führen. Jedoch zeigt Probe B experimentell keine PMA.

Die Erklärung für dieses Verhalten liegt in der mit Hilfe von EELS-Messungen ${ }^{5}$ beobachteten Bordiffusion (siehe Abbildung 4.9). An der Abbildung erkennt man, dass nach dem Auslagerungsschritt die Borkonzentration in der Tantal-Schicht deutlich erhöht ist. So muss während der Auslagerung Bor aus CoFeB in die Nachbarschichten der MTJ diffundieren [52], wodurch sich eine Grenzfläche aus CoFe/MgO bildet. Im Falle der Probe B, bei der es sich dabei um zwei MgO-Schichten handelt, kann Bor nur ins MgO-diffundieren oder sich an der CoFe/MgO-Grenzfläche sammeln. Dies beeinflusst die CoFe-Kristallisation und die Hybridisierung der Orbitale, was wiederum zur Absenkung des PMA-Wertes führt. Tantal nimmt während der Auslagerung die Boratome aus der CoFeB-Schicht auf und leitet eine ins Tantal gerichtete Bordiffusion ein. So sammeln sich die meisten Bor-Atome während der Auslagerung in der Tantalschicht. Die CoFe/MgO-Grenzschicht wird dadurch nicht beeinflusst. Dies führt zu einer an das $\mathrm{MgO}$ angepassten CoFeB-Kristallisation und als Folge zur Erhöhung der PMA. Außerdem erlaubt die Anpassung der Kristalle das kohärente Tunneln, das für den Spintransport von großer Bedeutung ist (siehe Unterabschnitt 2.2.3).

Insgesamt zeigen die von uns fabrizierten MTJs mit Tantal als Pufferschicht bis $\mathrm{zu}$ einer Schichtdicke von maximal $20 \mathrm{~nm}$ und einer Auslagerungstemperatur von

\footnotetext{
${ }^{5}$ Durchgeführt von Henning Schuhmann [51].
} 


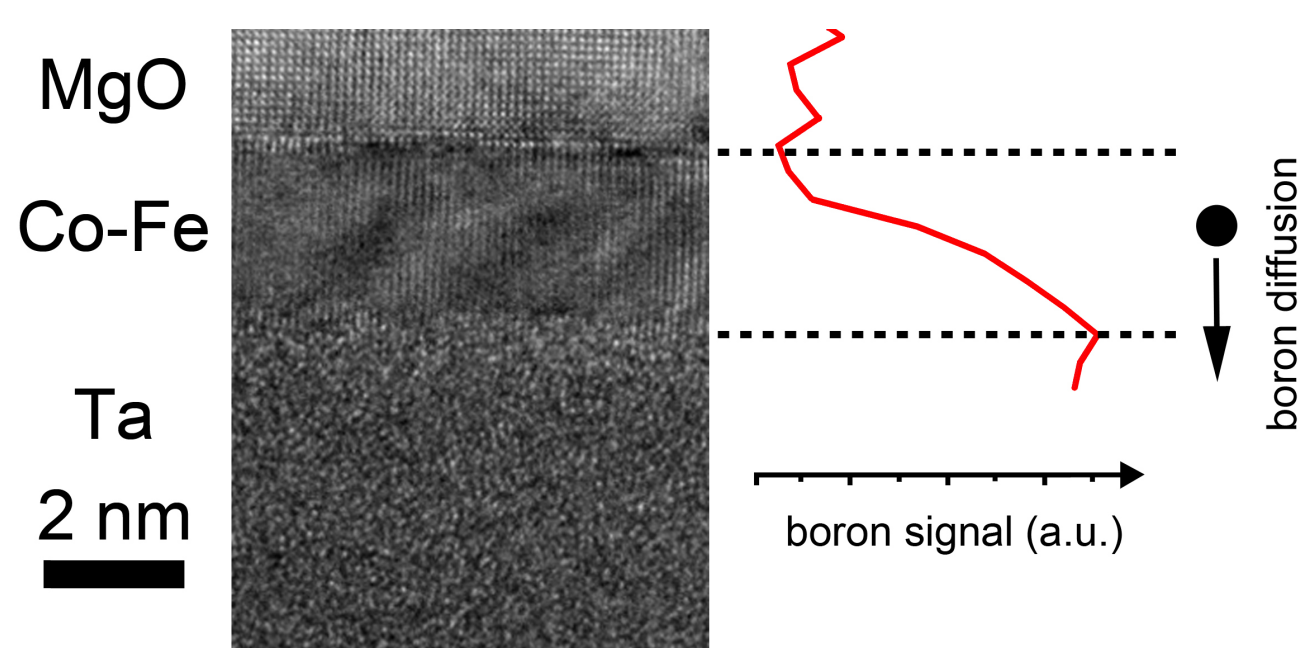

Abbildung 4.9.: EELS-Messung: (links) HRTEM-Aufnahme der Schichten, (rechts) an EELS gemessene Bor-Konzentrationsverteilung nach dem Annealing [50].

$300{ }^{\circ} \mathrm{C}$ eine Erhöhung von PMA gegenüber den MTJs ohne Tantal.

\subsection{Spin-transfer torque}

In diesem Kapitel wird zunächst die Qualität der hergestellten magnetischen Tunnelelemente diskutiert. Im Anschluss werden die ersten Spin-Transfer-Torque-Messungen an den Proben mit senkrechter Anisotropie gezeigt und diskutiert.

\subsubsection{Qualität der in- und out-of-plane Proben mit TMR als Qualitätsmerkmal.}

Im letzten Kapitel wurde der Einfluss einer Pufferschicht und der Dicke der ferromagnetischen Schicht auf das magnetische Verhalten der Tunnelelemente diskutiert. Ein weiterer Parameter, der einen essentiellen Einfluss auf die Qualität der Tunnelelemente hat, ist die Barriere. In unserer Gruppe wird eine kristalline (100) MgOSchicht als Barriere verwendet. Bei einer ausreichenden Anpassung der Kristallgitter der CoFeB- und MgO-Schichten aneinander, wird das kohärente Tunneln möglich (Unterabschnitt 2.2.3). Dies hat einen direkten Einfluss auf das TMR-Verhalten: Das kohärente Tunneln der Elektronen ist eine Voraussetzung für ein hohes TMR. Umgekehrt ist ein hoher TMR-Wert ein Hinweis auf das kohärente Tunneln bzw. auf die Qualität der MgO-Barriere.

Zur Abschätzung der Qualität der Barriere werden zwei Proben mit Standardschichtstapeln für in-plane und out-of-plane Anisotropie betrachtet. Die Zusammensetzung der Proben ist an der Abbildung $4.10 \mathrm{zu}$ sehen. Die Barrierendicke der PMA-Probe und die Tunnelelementgröße wurden kleiner gewählt, weil in der Regel 


\begin{tabular}{|c|c|}
\hline $\mathrm{Ru} 3 \mathrm{~nm}$ & $\mathrm{Ru} 3 \mathrm{~nm}$ \\
\hline Ta $5 \mathrm{~nm}$ & Ta $5 \mathrm{~nm}$ \\
\hline CoFeB $5.4 \mathrm{~nm}$ & CoFeB $1.2 \mathrm{~nm}$ \\
\hline $\mathrm{MgO} 2.1 \mathrm{~nm}$ & $\mathrm{MgO} 0.84 \mathrm{~nm}$ \\
\hline CoFeB $2.5 \mathrm{~nm}$ & CoFeB $1 \mathrm{~nm}$ \\
\hline Ta $15 \mathrm{~nm}$ & $\mathrm{Ta} 15 \mathrm{~nm}$ \\
\hline SiOx - Sub & SiOx - Sub \\
\hline
\end{tabular}

Abbildung 4.10.: Standardstapel: (a) für Proben mit in-plane Anisotropie und (b) für Proben mit out-of-plane Anisotropie.

ein niedrigerer Widerstand und kleinere Fläche des Tunnelelements für eine Absenkung der Schaltströme sorgen. An beiden Proben wurde der TMR-Effekt vermessen. Die Ergebnisse der Messungen sind in Abbildung 4.11 und Abbildung 4.12 zu sehen. Diese Kurven stellen die höchsten gemessenen Werte, der hier benutzten Proben, für jeweilige Zusammensetzung und Ausrichtung der Anisotropie dar. Tunnelelemente mit gleicher Zusammensetzung zeigen ähnliches Verhalten der TMR-Kurven, auch wenn der TMR-Wert niedriger liegt.

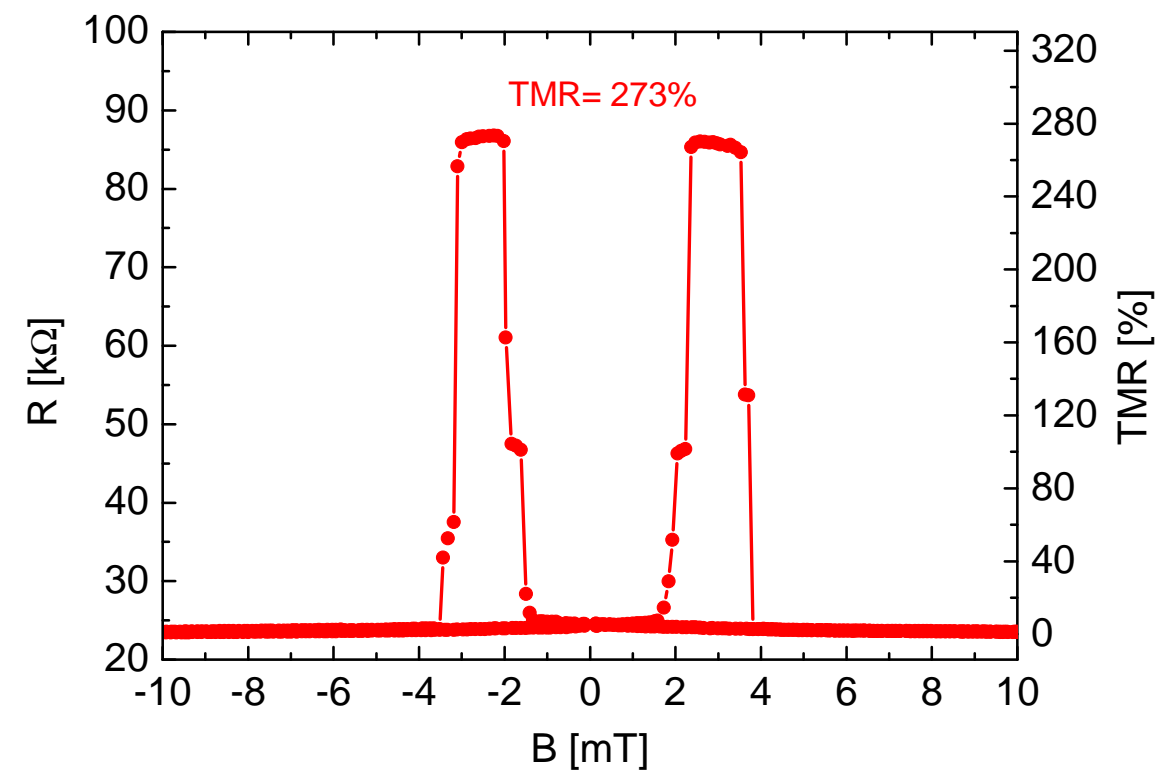

Abbildung 4.11.: In-plane TMR Wert für Standardschichtenstapel: SiOx/ Ta 15 $\mathrm{nm} / \mathrm{CoFeB} 2,5 \mathrm{~nm} / \mathrm{MgO} 2,1 \mathrm{~nm} / \mathrm{CoFeB} 5,4 \mathrm{~nm} / \mathrm{Ta} 5 \mathrm{~nm} / \mathrm{Ru} 3 \mathrm{~nm}$.

In der Tabelle 4.3 sind die zusammengefassten Eigenschaften der in- und outof-plane TMR-Werte. Der PMA-TMR-Wert liegt niedriger als der in-plane Wert, 


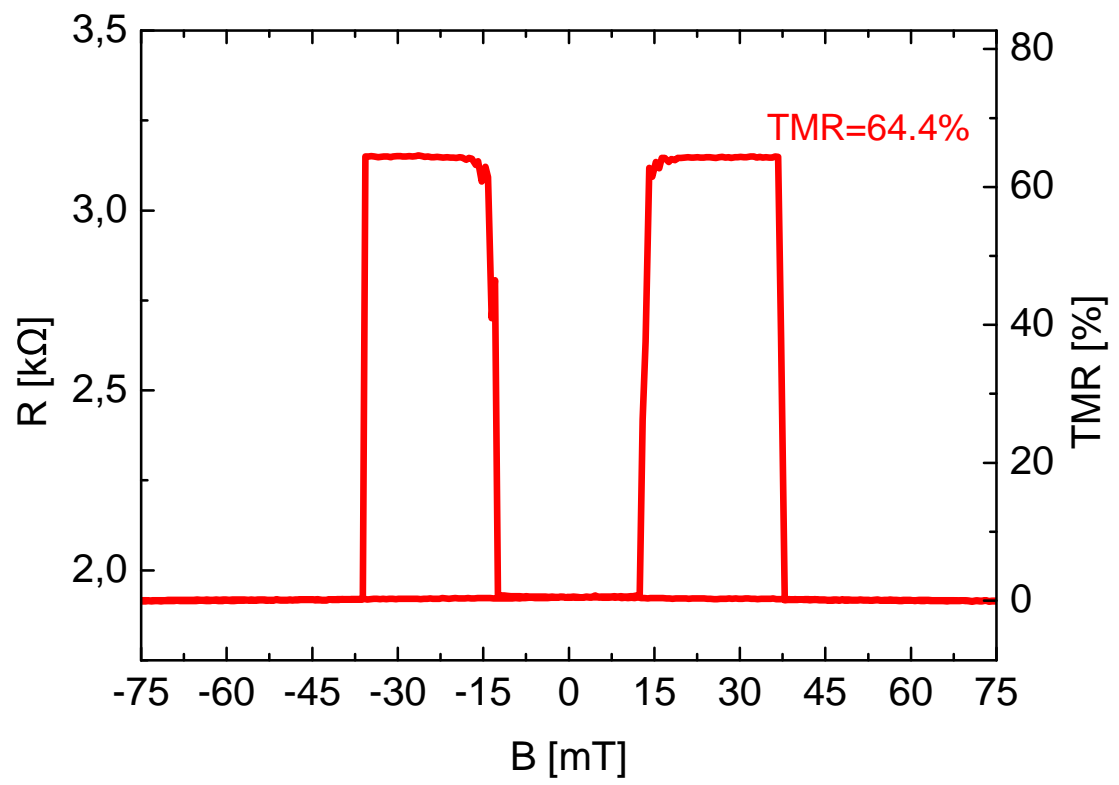

Abbildung 4.12.: Out-of-plane TMR Wert für Standardschichtenstapel: $\mathrm{SiOx} / \mathrm{Ta}$ $15 \mathrm{~nm} / \mathrm{CoFeB} 1 \mathrm{~nm} / \mathrm{MgO}$ 0,84 nm / CoFeB 1,2 nm / Ta $5 \mathrm{~nm} / \mathrm{Ru} 3 \mathrm{~nm}$.

\begin{tabular}{|c|c|c|}
\hline & out-of-plane & in-plane \\
\hline \hline Schichtdicke [nm] & $<1,35$ & $>1,35$ \\
\hline MgO-Schicht [ML] & $3-7$ & $8-12$ \\
\hline Max. TMR & $64,4 \%$ & $280 \%$ \\
\hline Min. TMR & $<3 \%$ & $<3 \%$ \\
\hline Annealing & $300{ }^{\circ} \mathrm{C}$ & $450{ }^{\circ} \mathrm{C}$ \\
\hline
\end{tabular}

Tabelle 4.3.: Vergleich der in- und out-of-plane Tunnelelemente.

jedoch wird bei PMA-Elementen eine dünnere MgO-Barriere verwendet, um den Widerstand für weitere Experimente möglichst niedrig zu halten. Eine dünnere Barriere hat natürlich einen abgeschwächten Spin-Filter-Effekt, was zur Abnahme des TMR-Effektes führt. Der TMR-Effekt von $64,4 \%$ bei einer 5 Monolagen dünnen $\mathrm{MgO}$-Schicht und das Verhalten der TMR-Kurven (mit rechteckigen Plateaus) sprechen für eine gute Qualität der Barriere. Die in-plane TMR-Werte von ca. 280\% sind relativ hoch und sind mit den Werten der anderen Forschungsgruppen vergleichbar: z. B. in den Arbeitsgruppen von A. Thomas und G. Reiss aus Bielefeld wurde einen TMR von ca. $330 \%$ bei Raumtemperatur gemessen [53].

Die Qualität der Proben kann man auch durch die Untersuchung der IU-Kennlinien abschätzen. An der Abbildung 4.13 sieht man die IU-Kennlinien, die für jeweils parallele und antiparallele Magnetisierung der MTJs aufgenommen wurden. Wird an den Kennlinien die Brinkman-Anpassung gemacht, so liefert sie Parameter, die in 

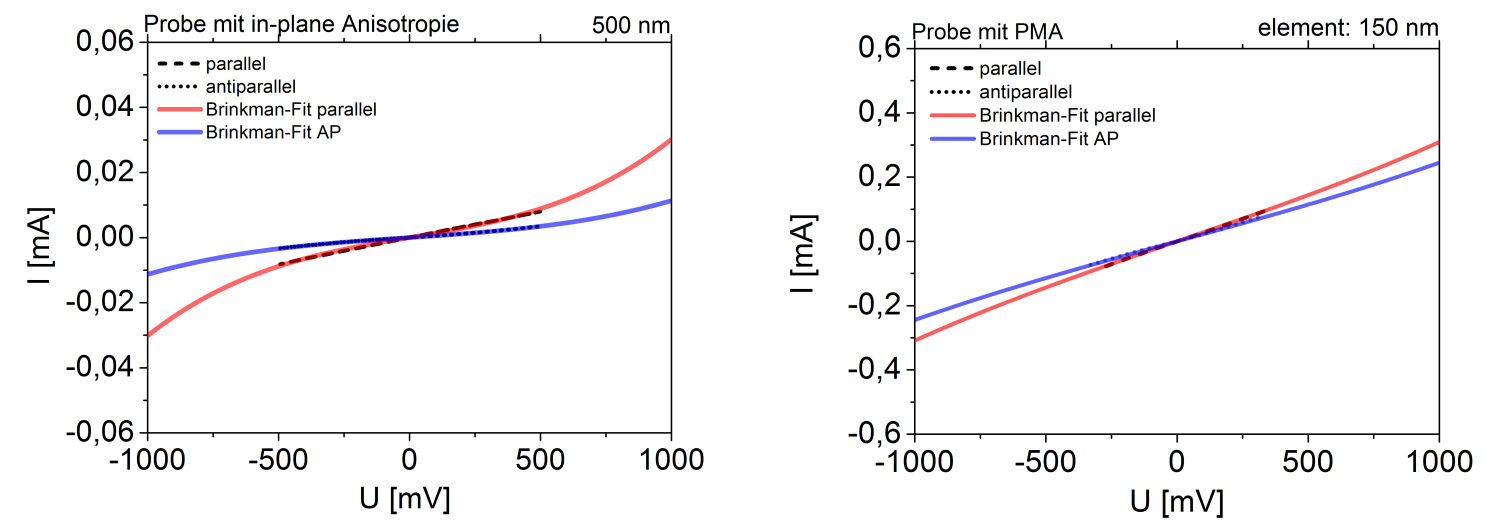

Abbildung 4.13.: IU-Kennlinien und die entsprechenden Brinkmann-Fits der Probe mit in-plane Anisotropie (links) und der Probe mit out-of-plane Anisotropie (rechts).

\begin{tabular}{|c|c|c|c|}
\hline & In-plane & Out-of-plane & \multirow{2}{*}{ Fit Parameter } \\
\hline & $\mathrm{AP} \quad \mathrm{P}$ & $\mathrm{AP} \quad \mathrm{P}$ & \\
\hline$d_{f i t}[\AA]$ & 21 & 8,4 & fest \\
\hline$\varphi_{f i t}[\mathrm{eV}]$ & \begin{tabular}{l|l}
$1,717(6)$ & $1,532(1)$
\end{tabular} & \begin{tabular}{|l|l|}
$3,109(3)$ & $2,945(5)$ \\
\end{tabular} & frei \\
\hline$c_{f i t}\left[m_{e}\right]$ & 0,39 & 0,39 & fest \\
\hline TMR [\%] & $210-220$ & $8-30$ & - \\
\hline
\end{tabular}

Tabelle 4.4.: Parameter und Ergebnisse des Brinkmann Fits. Als „festen Parameter" bezeichnet man den vorgegebenen Parameter.

der Tabelle 4.4 aufgeführt sind. Von besonderem Interesse sind die Werte für die Barrierenhöhe $\varphi$ in eV. Als vorgegebene Parameter wurden folgende Größen verwendet: effektive Masse $m^{*}=0,39 m_{e}$ (dieser Wert wurde in [15] ermittelt), $A$ Fläche des Elements in $\mu^{2}, \Delta \varphi$ wurde zunächst auf $0 \mathrm{eV}$ gesetzt ${ }^{6}$ und $d$ entspricht der aufgedampften Barrierendicke der Elemente in Ångström.

Die Kurven des Brinkman-Fits sind in Abbildung 4.13 abgebildet. Die Krümmung der Anpassungskurven setzt erst bei ca. $0,75 \mathrm{~V}$ für die in-plane Probe und bei etwa $1 \mathrm{~V}$ für die out-of-plane Probe ein. Die Kennlinien wurden aufgrund der Empfindlichkeit der Elemente (siehe Unterabschnitt Thermische Stabilität) nur für den Messbereich von ca. $[-0,5 \mathrm{~V} ; 0,5 \mathrm{~V}]$ aufgenommen. Außerdem zeigt das BrinkmanModell nur für Proben mit einer dicken $(d>1 \mathrm{~nm})$ Barriere zuverlässige Vorhersagen [15]. Jedoch wird der Brinkman-Anpassung an dieser Stelle nur zur groben Abschätzung und Vergleich der Tunnelbarrierenqualität verwendet.

Vergleicht man die Proben mit IPA und $\mathrm{PMA}^{7}$, so zeigt die PMA-Probe eine Barrierenhöhe $\varphi$, die ca. um den Faktor 2 höher als der Wert der IPA-Probe liegt.

\footnotetext{
${ }^{6}$ Wenn der Fit an einer Hälfte nicht passte, so wurde $\triangle \varphi$ als freier Parameter gesetzt.

${ }^{7}$ In-Plane Anisotropy (IPA) und Perpendicular Magnetic Anisotropy (PMA).
} 


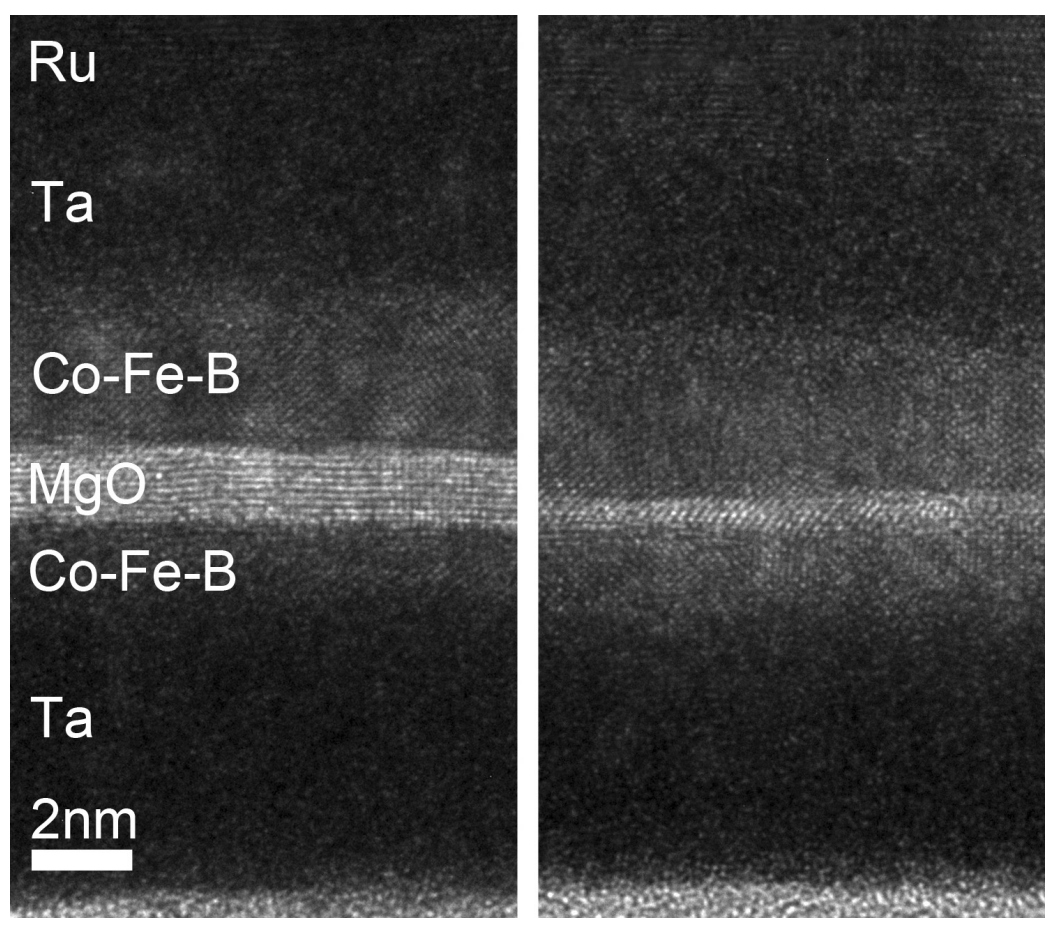

Abbildung 4.14.: HRTEM-Aufnahme der MTJs mit einer 10 Monolagen dicken MgO-Barriere (links) und einer 3 Monolagen dicken MgO-Barriere (rechts) [54].

Werte mit $\varphi>2 \mathrm{eV}$ sind für eine kristalline $\mathrm{MgO}$-Barriere untypisch, da sie bereits mit den Erwartungen für amorphe Barrierenhöhen vergleichbar sind [15]. Die aus dem Brinkman-Fit berechneten Barrierenhöhen für die Probe mit PMA sprechen für eine deutlich niedrigere Qualität der Barriere im Vergleich zu Proben mit IPA. Dies entspricht der Erwartung, da die MgO-Schichtdicke der in-plane Probe mehr als doppelt so hoch ist. Dadurch erhöhen sich die Spinfilter-Eigenschaften um mehrere Größenordnungen. Auch der gemessene TMR-Effekt zeigt eine deutliche Abnahme für die dünnere Barriere. Der erwartete Unterschied der Barrierenhöhen zwischen AP- und P-Zuständen ist auch deutlich erkennbar.

Zur weiteren Untersuchung der MgO-Barriere und der Kristallisation der CoFeBSchicht wurden HRTEM-Aufnahmen von den magnetischen Tunnelelementen durchgeführt. Das Ergebnis ist an der Abbildung $4.14 \mathrm{zu}$ sehen [54]. Die 10 Monolagen (kurz ML) dicke MgO-Schicht zeigt eine ausgeprägte Kristallisation. Die beiden CoFeB-Schichten zeigen auch eine Kristallstruktur. Dies ermöglicht das kohärente Tunneln der Proben, was sich in einem relativ hohen TMR-Wert von bis zu $280 \%$ widerspiegelt. Die dünnere Barriere mit der nur 3 ML dicken MgO-Schicht zeigt an der HRTEM-Aufnahme eine Kristallstruktur, die sich an die CoFeB-Schichten überträgt. Die Qualität der Schicht soll auch das kohärente Tunneln ermöglichen. Die höchsten TMR-Werte der Proben mit PMA liegen bei ca. 65\%. An dieser Stelle muss betont werden, dass der niedrige TMR-Effekt im Vergleich zur in-plane Probe keineswegs an der Qualität der Barriere liegt, sondern an der Barrierendicke. Da die 
Barrierendicke der MgO-Schicht essentiell für Spinfilter-Eigenschaften ist, wird auch der TMR-Wert mit einer dünneren Barriere kleiner. Jedoch wird der Widerstand der Proben auch kleiner, was von Bedeutung bei der Spin-Transfer-Torque-Messung ist. Da der präsentierte Schichtenstapel speziell für diese Messungen optimiert wurde, musste die Barrierendicke zugunsten des niedrigeren Widerstands reduziert werden.

\subsubsection{Spin-transfer torque an Tunnelelementen mit PMA}

Zur weiteren elektrischen Charakterisierung wurden IR-Kennlinien der magnetischen Tunnelelemente aufgenommen. Die Messungen wurden an der folgenden Probe durchgeführt: SiOx-Sub / $15 \mathrm{~nm}$ Ta / 1,1 nm CoFeB / 0,84 nm MgO / 1,32 nm $\mathrm{CoFeB} / 5 \mathrm{~nm} \mathrm{Ta} / 3 \mathrm{~nm} \mathrm{Ru}$. Diese Probe wurde nach dem Herstellen eine Stunde lang ex-situ bei $T=300^{\circ} \mathrm{C}$ ausgelagert.

Es wurden an jedem Element möglichst viele Messungen (> 150) durchgeführt, um die statistische Verteilung der kritischen Schaltströme berechnen zu können. Dabei wurde zunächst eine TMR-Kurve aufgenommen. Aus der TMR-Kurve wurden die Magnetfeldwerte für AP- und P-Zustände ermittelt. Vor der Aufnahme der IR-Kennlinie wurde das Magnetfeld vom parallelen Zustand (B-Feld negativ) zum antiparallelen Zustand (B-Feld positiv) umgeschaltet. Damit wurde zum Beginn der Messung ein AP-Zustand mit einem positiv eingestellten Magnetfeld erzeugt. Bei der Aufnahme der IR-Kennlinie wurde das Magnetfeld nicht mehr verändert, so dass die Schaltvorgänge nur durch Stromänderung hervorgerufen worden sind.

Eine typische Messkurve aus fünf wiederholten Messungen ist in Abbildung 4.15 zu sehen. Die Schaltströme variieren unkorreliert mit jeder Messung, so dass man nur von einem Schaltstrombereich $\triangle j$ reden kann. Im Folgenden werden die kritischen Schaltströme für zwei kreisförmige Elemente bestimmt: mit einem Durchmesser von $150 \mathrm{~nm}$ und einem Durchmesser von $500 \mathrm{~nm}$. 


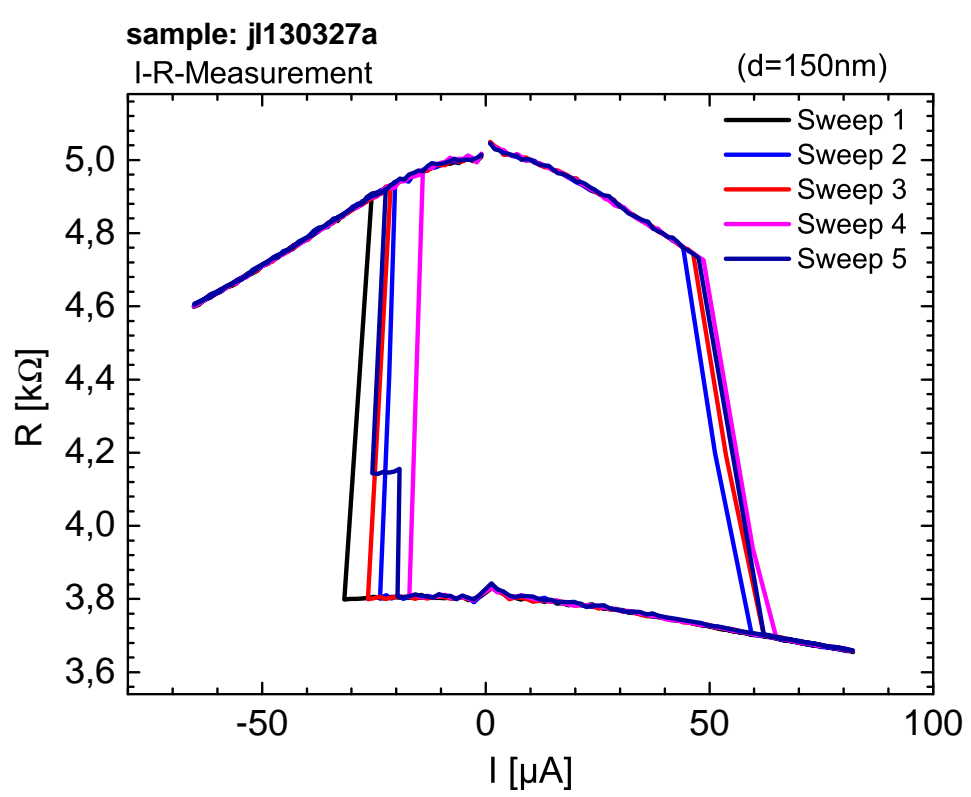

Abbildung 4.15.: IR-Kennlinie des $150 \mathrm{~nm}$ breiten Elements für fünf nacheinander ausgeführte Messungen.

In Abbildung 4.16 (a) und (b) sind die statistischen Verteilungen der Schaltströme $^{8}$ für die jeweiligen $P \rightarrow A P$ und $A P \rightarrow P$ Übergänge des Elements mit kleinerem Durchmesser $(150 \mathrm{~nm})$ abgebildet. $\triangle j$ entspricht der Halbwertsbreite der kritischen Stromstärke, $I_{c}$ entspricht dem kritischen Schaltstrom. Die Abbildung 4.15 (c) und (d) entsprechen der Wahrscheinlichkeit, dass das Element zum gegebenen Strom bereits von $P \rightarrow A P$ oder von $A P \rightarrow P$ geschaltet hat. Diese Auftragung lässt die Möglichkeit auch zur gegebenen Wahrscheinlichkeit die Stromstärke zur ermitteln. Die Schaltstromstatistik für das Element mit $500 \mathrm{~nm}$ Durchmesser ist in Abbildung 4.17 zu sehen. Die $\triangle j$ für das Element mit einem größeren Durchmesser $(500 \mathrm{~nm})$ wurde analog zu dem kleineren Element mit einem Gauß-Fit ermittelt. Die für die beiden Elemente relevante Werte wurden aus beiden Abbildungen ermittelt und sind in der Tabelle 4.5 dargestellt.

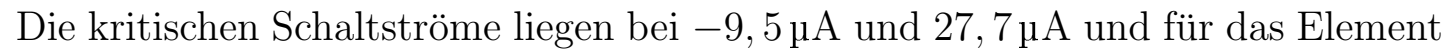
mit $150 \mathrm{~nm}$ Durchmesser. Für das Element mit einem $500 \mathrm{~nm}$ Durchmesser liegen die kritischen Schaltströme bei $-5 \mu \mathrm{A}$ und $42 \mu \mathrm{A}$. Diese Ströme entsprechen der $95 \%$ Wahrscheinlichkeit für das Schalten der Elemente. Wenn diese Ergebnisse mit den Messungen aus [48] vergleichen werden, die an ähnlichen Proben, jedoch an kleineren Elementen (Durchmesser $70 \mathrm{~nm}$ ) durchgeführt worden sind (siehe Abbildung 4.18), dann lässt sich leicht erkennen, dass die von H. Sato et al. [48] gemessenen Ströme (gemittelt über beide Tunnelelemente) um ca. einen Faktor ${ }^{9} 13$ für den AP-P-

\footnotetext{
${ }^{8}$ Bei (a) und (b) sind die relativen Häufigkeiten aufgetragen. 100\% wären in diesem Fall alle durchgeführten Messungen.

${ }^{9}$ Für den Faktor $f$ gilt: $I_{\text {Krit }}($ Sato $)=f \cdot I_{\text {Krit }}($ Messung $)$.
} 

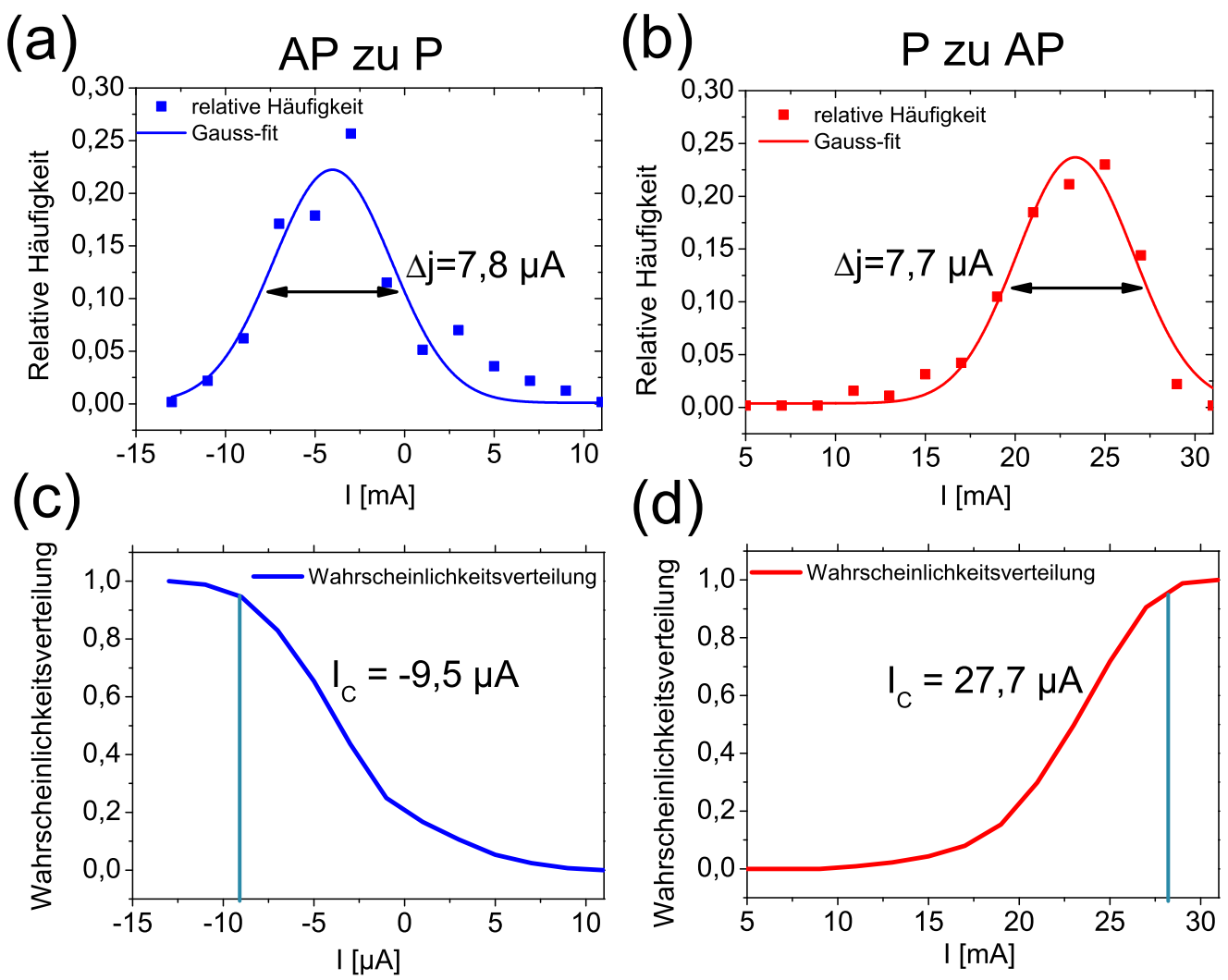

Abbildung 4.16.: Schaltstromstatistik an dem Tunnelelement mit $150 \mathrm{~nm}$ Durchmesser. (a) und (b) zeigen die relativen Häufigkeitsverteilungen der Schaltströme für die Übergänge $\mathrm{AP} \rightarrow \mathrm{P}$ und $\mathrm{P} \rightarrow \mathrm{AP}$. (c) und (d) sind die entsprechenden Wahrscheinlichkeitsverteilungen. Als kritischen Strom wird der Schaltstrom definiert, bei dem die Wahrscheinlichkeit des AP-P-Übergangs mindestens $95 \%$ beträgt.

Übergang und um ca. einen Faktor 5 für den P-AP-Übergang höher als unsere Werte liegen (also durchschnittlich Unterschied um den Faktor 9). Die Schaltströme des Elements mit dem $500 \mathrm{~nm}$ Durchmesser liegen in der gleichen Größenordnung wie die Ströme des kleineren Elements. Beim P-AP-Übergang zeigt das Element mit größerem Durchmesser (500 nm) einen im Vergleich zum kleineren Element höheren Wert für den kritischen Strom von $42 \mu \mathrm{A}$. Insgesamt liegen die kritischen Ströme der in dieser Arbeit untersuchten Elemente unter den von H. Sato et al. [55] berichteten. Die kleineren Schaltströme sind für die Anwendungen vom besonderen Interesse, da sie aufgrund ihres niedrigeren Energieverbrauchs ökonomischer sind. Jedoch kann der kleinere Schaltstrom bei größeren Elementgrößen für eine niedrigere thermische Stabilität sprechen. Unter diesem Blickwinkel wird die thermische Stabilität $\triangle$ im nächsten Unterabschnitt berechnet und diskutiert. 
(a)
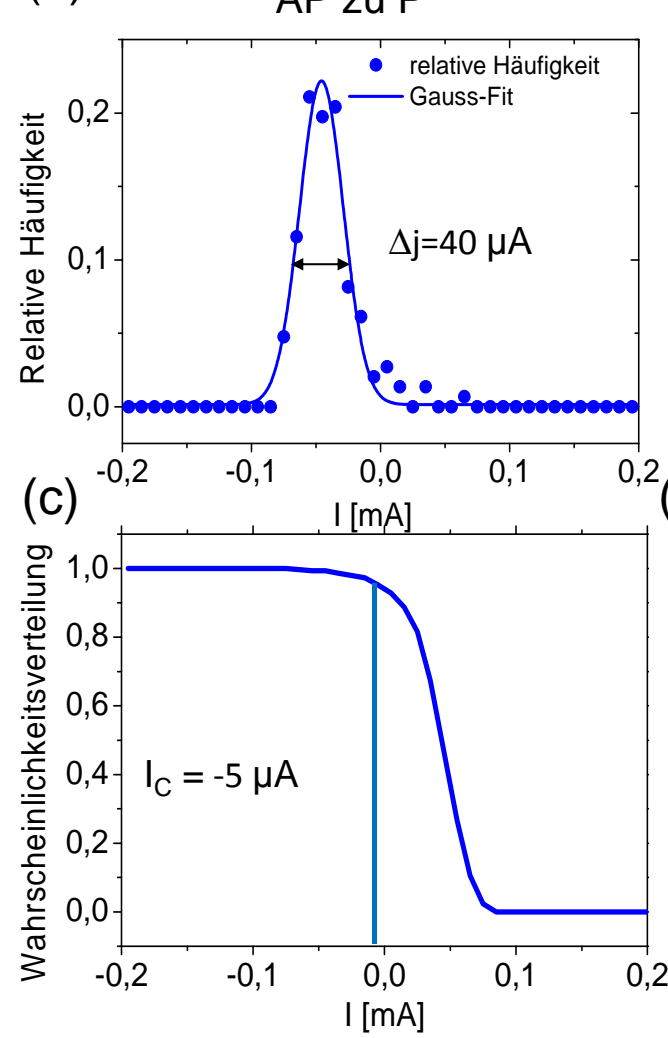

(b)

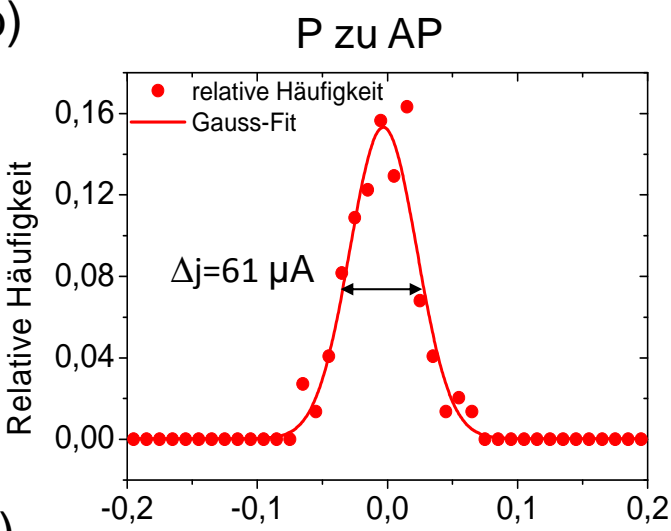

(d)

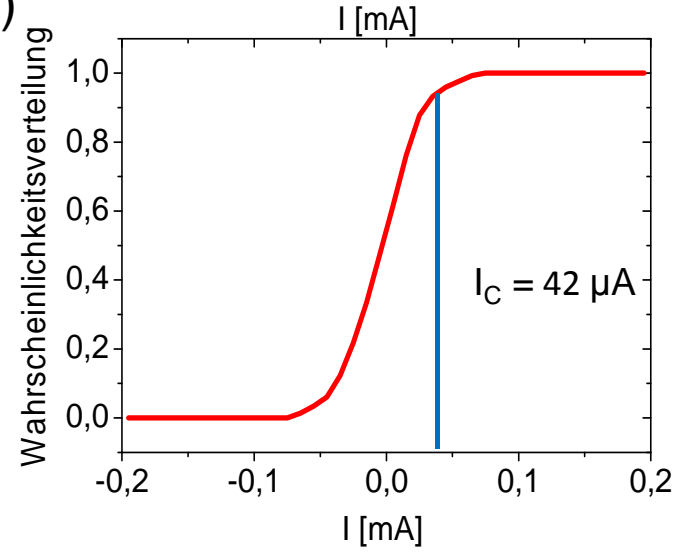

Abbildung 4.17.: Schaltstromstatistik an dem Tunnelelement mit $500 \mathrm{~nm}$ Durchmesser. Die oberen Grafiken (a) und (b) zeigen die relativen Häufigkeiten der aufgetretenen Schaltströme. Die unteren Grafiken (c) und (d) zeigen die Wahrscheinlichkeitsverteilungen für entsprechende Stromstärken das Element zu schalten.

\begin{tabular}{|c|c|c|c|c|}
\hline & AP-P & P-AP & AP-P & P-AP \\
\hline & $150 \mathrm{~nm}$ & $150 \mathrm{~nm}$ & $500 \mathrm{~nm}$ & $500 \mathrm{~nm}$ \\
\hline \hline$I_{\text {krit }}[\mu \mathrm{A}]$ & $-9,5$ & 27,7 & $-5,2$ & 41,9 \\
\hline$\triangle I[\mu \mathrm{A}]$ & 7,8 & 7,9 & 40 & 61 \\
\hline
\end{tabular}

Tabelle 4.5.: Ergebnisse für den kritischen Strom, Strombreite und Stromdauer, bei der die Schaltwahrscheinlichkeit von ca. $30 \%$ auf ca. $80 \%$ steigt (also um 50 $\%$ ansteigt). 


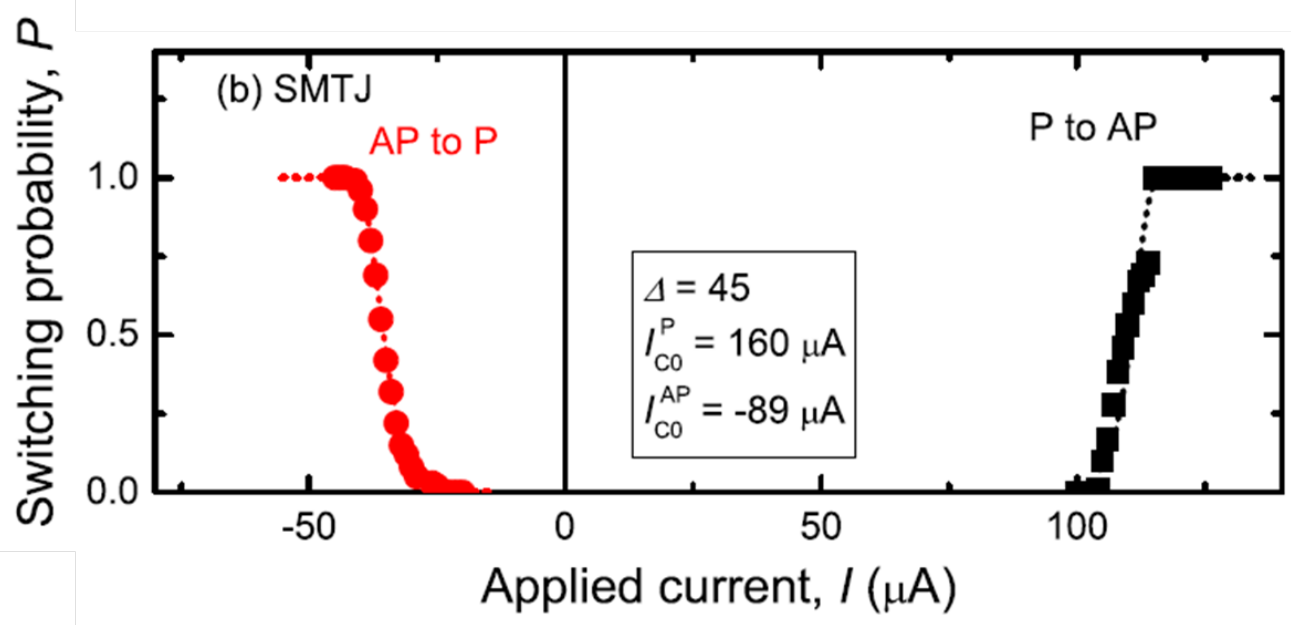

Abbildung 4.18.: Messungen der Gruppe H. Sato et al [55].

\subsubsection{Thermische Stabilität}

Zur Untersuchung der thermischen Stabilität wurde folgende Formeln verwendet $[48,55]$ :

$$
P_{P(A P)}=1-\exp \left[-\frac{\tau_{p}}{\tau_{0}} \exp \left\{-\triangle_{P(A P)}\left(1-\frac{I}{I_{\text {crit }}^{P(A P)}}\right)\right\}\right]
$$

$P$ entspricht der Wahrscheinlichkeitsverteilung, $\tau_{0}-$ die Schaltdauer ${ }^{10}$, I ist der Strom, $\tau_{p}-$ Strompulsdauer.

Mit der Gleichung 4.2 ist die Berechnung der thermischen Stabilität $\triangle$ aus den Abbildung 4.16 und Abbildung 4.17 (jeweils (a) und (b)) möglich. Dafür muss nur die Wahrscheinlichkeitsverteilung angepasst werden. Lässt man $\triangle$ als freien Parameter stehen, so wird diese durch die Anpassung (engl. Fit) bestimmt. Die Ergebnisse für entsprechende Wahrscheinlichkeitsverteilungen sind in der Tabelle 4.6 zu sehen. Die in der Tabelle gezeigte thermische Stabilität stellt einen Mittelwert aus $\triangle_{P \rightarrow A P}$ und $\triangle_{A P \rightarrow P}$ dar.

Die Werte für die thermische Stabilität $\triangle$ der hier benutzten Elemente liegen deutlich niedriger als von H. Sato et al. [48] berichtet wurde. Die Faktoren $\Lambda_{\triangle}=$ $\frac{\triangle_{\text {Sato }}}{\triangle_{\text {Messung }}}$ um die sich die Werte unterscheiden sind in der Tabelle $4.6 \mathrm{zu}$ sehen. Dies könnte auch die deutlich niedrigeren Schaltstromwerte erklären: durch die kleinere thermische Stabilität der Elemente werden auch kleinere Ströme zum Schalten der MTJs benötigt. Insgesamt wenn man den Unterschied der kritischen Schaltströme und der thermischen Stabilität der MTJs mit den Ergebnissen aus [48, 55] vergleicht, so zeigen die in dieser Arbeit benutzten Proben einen deutlichen Trend: durch klei-

\footnotetext{
${ }^{10}$ Wird in der Anpassung als freie Variable behandelt.
} 


\begin{tabular}{|c|c|c|c|}
\hline Probe & Durchmesser $[\mathrm{nm}]$ & $\triangle$ & $\Lambda_{\triangle}$ \\
\hline \hline Messung & 150 & $9,2 \pm 1,6$ & $\sim 4,9$ \\
\hline Messung & 500 & $15,5 \pm 0,7$ & $\sim 2,9$ \\
\hline H. Sato et al. & 70 & 45 & 1 \\
\hline
\end{tabular}

Tabelle 4.6.: Berechnete thermische Stabilität und der Unterschiedsfaktor zur Ergebnissen von H. Sato et al. Verglichen werden in unserer Gruppe hergestellten und von Johannes Christian Leutenantsmeyer vermessene Proben.

nere thermische Stabilität konnten die Schaltströme um ca. den Faktor 9 abgesenkt werden.

Die thermische Stabilität zeigt eine in der Literatur $[48,55]$ berichtete Abhängigkeit von der Elementgröße. So weisen größere Elemente eine höhere thermische Stabilität auf: $\triangle(150 \mathrm{~nm}) \approx 9$ und $\triangle(500 \mathrm{~nm}) \approx 16$ auf. Dies liegt daran, dass thermische Stabilität von dem Volumen der Elemente abhängig ist und somit mit steigendem Volumen größer wird.

Für die späteren Anwendungen der STT-Elemente ist ein Kompromiss zwischen möglichst niedrigen Schaltströmen und einer möglichst hohen thermischen Stabilität von Bedeutung. Zur Verbesserung der thermischen Stabilität wurde von H. Sato et al. [48, 55] eine Änderung an dem Schichtenstapel vorgeschlagen: in eine der beiden CoFeB-Schichten kann eine sehr dünne $(\leq 0,4 \mathrm{~nm})$ Tantal-Schicht eingebaut werden. Dies kann zu einer weiteren Borabnahme an den CoFeB- und MgO-Schichten führen. Eine bessere Kristallisation und Anpassung der MgO- und CoFeB-Schichten aneinander wäre die Folge (siehe dazu auch Abschnitt 5.2).

\subsection{Dynamik an MTJs mit PMA}

In diesem Abschnitt wird der Spintransport in magnetischen Tunnelelementen auf ultrakurzen Zeitskalen untersucht und diskutiert. Im ersten Unterabschnitt wird die Vorarbeit präsentiert, in welcher durch Laser-Pulse angeregte Spinströme in einer Eisenschicht mit unterschiedlichen Deckschichten untersucht wurden. Dies leitet zu den hier im Folgenden untersuchten Tunnelstrukturen über.

Im zweiten Unterabschnitt wird kurz auf das Verhalten der parallelen und antiparallelen Zustände der magnetischen Tunnelelemente in Abhängigkeit von der Dicke des Ferromagneten eingegangen. Auf ultrakurzen Zeitskalen wird hier der Spintransport, manipuliert durch die parallele und antiparallele Orientierung, untersucht.

Der dritte Unterabschnitt behandelt das magnetische Verhalten der MTJs unter Einfluss von Laserpulsen. Die möglichen Spintransportmechanismen werden diskutiert. 


\subsubsection{Spinstrompulse}

In diesem Unterabschnitt wird die für diese Dissertation wichtige Vorarbeit [38], die aus einer Kollaboration mit der Arbeitsgruppe von Tobias Kampfrath entstand, kurz zusammengefasst wiedergegeben.

a

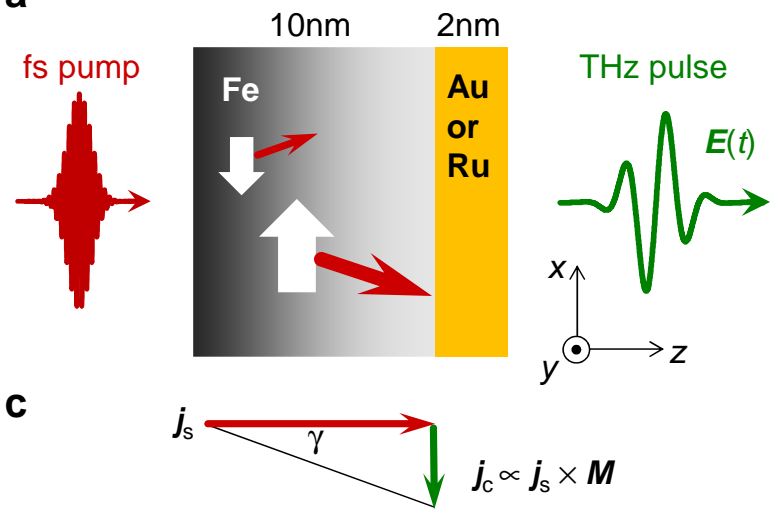

b

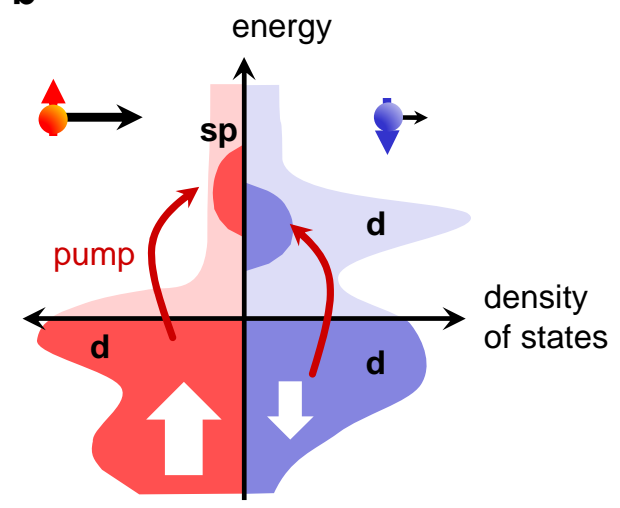

Abbildung 4.19.: (a) Skizze des Experiments: ein fs-Laserpuls trifft eine $10 \mathrm{~nm}$ dicke Eisenschicht mit einer $\mathrm{Au}$ oder $\mathrm{Ru}$ Deckschicht. (b) Schema der Energiebänder der Eisenschicht. (c) Inverser Spin-Hall Effekt: die Majoritäts- und Minoritätselektronen werden in unterschiedliche Richtungen abgelenkt. [38]

Die Grundidee des folgenden Experiments ist in Abbildung 4.19 skizziert: eine $10 \mathrm{~nm}$ dicke Eisenschicht mit einer Ru- oder Au-Deckschicht wird von einem fsLaserpuls (Dauer $20 \mathrm{fs}$ ) getroffen. Die Photonenenergie liegt bei 1,55 eV. Der Laserpuls regt die Elektronen aus der Eisenschicht an und bringt sie auf die Energieniveaus, die über der Fermi-Energie liegen. Die Majoritätselektronen der Eisenschicht werden dabei auf das sp-Niveau (siehe Abbildung 4.19 (b)) angeregt und die Minoritätselektronen auf das d-Energieband. Die Geschwindigkeit der angeregten Elektronen unterscheidet sich stark abhängig vom jeweiligen Energieband. So sind die Elektronen aus dem sp-Band ca. 5 mal schneller als Elektronen aus dem d-Band [39]. Je nach der Deckschicht besetzen die angeregten Elektronen unterschiedliche Bänder in der Au- oder Ru-Schicht: In Gold werden die schnelleren (mit einer Geschwindigkeit von ca. $1 \frac{n m}{f s}$ ) und langlebigen (Lebenszeit $100 \mathrm{fs}$ ) sp-Niveaus besetzt [39]. In Ruthenium dagegen wird dagegen das langsamere d-Niveau $\left(\sim 0,1 \frac{\mathrm{nm}}{\mathrm{fs}}\right)$ besetzt [56]. So entsteht durch angeregte Elektronen in Ruthenium ein deutlich langsamerer und Spinträger reicherer Transport als in der Goldschicht. Dies wurde bereits experimentell nachgewiesen (siehe Abbildung 4.20). Die weiteren Details zu dem Experiment sind in [38] genauer beschrieben.

Die Eigenschaft der Eisenschicht durch einen Laserpuls zwei von der Geschwindigkeit und vom Spin unterschiedliche Stromflüsse zu generieren, ist von Bedeutung in den nächsten Kapiteln dieser Arbeit. 


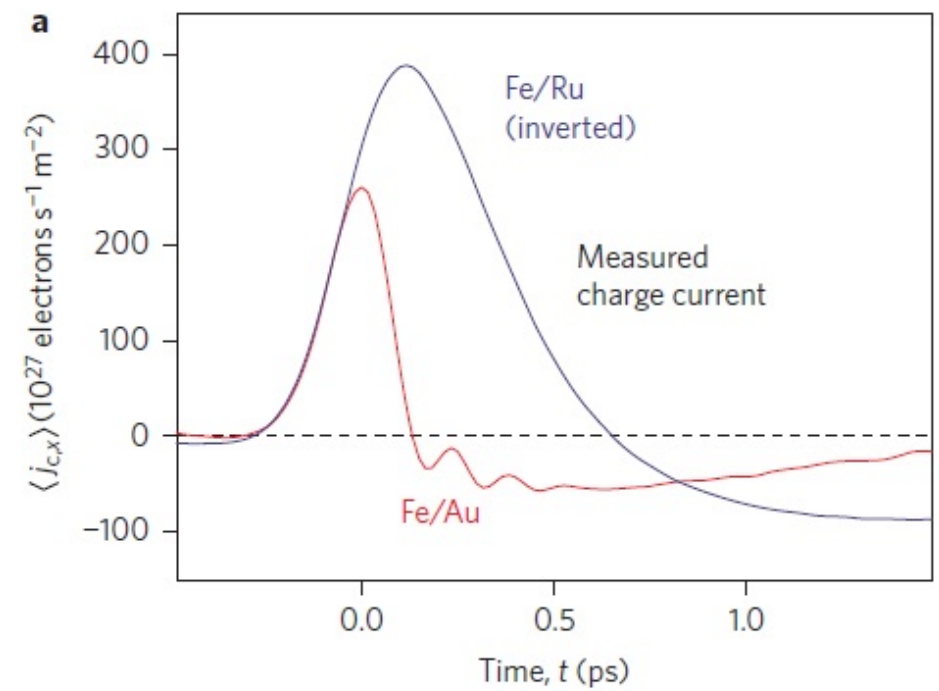

Abbildung 4.20.: Experimentell bestimmte in-plane Stromdichte. Die blaue Kurve wurde zur besseren Vergleichbarkeit auf der y-Achse invertiert [38].

\subsubsection{Magnetisches Schalten der MTJs}

In den nächsten Unterabschnitten werden Messungen an den bereits beschriebenen MTJs auf ultrakurzen Zeitskalen gezeigt und diskutiert. Der Schichtenstapel ist wie folgt aufgebaut: SiOx-Substrat / $15 \mathrm{~nm} \mathrm{Ta} /$ 0,9 nm CoFeB / 1,26 nm MgO / 1,2 $\mathrm{nm} \mathrm{CoFeB} / 2 \mathrm{~nm} \mathrm{Ta} / 2 \mathrm{~nm} \mathrm{Ru}$. Die Probe wurde für eine Stunde bei $300^{\circ} \mathrm{C}$ ohne externes Magnetfeld ausgelagert.

Das Interesse bei den Messungen bestand darin, den parallelen und antiparallelen Zustand der MTJs auf ultrakurzen Zeitskalen zu untersuchen und nach einem Unterschied im Verhalten der Demagnetisierungskurven zu suchen. Zu diesem Zweck wurden als Vorarbeit einige Probenreihen vermessen und optimiert. Die Zielsetzung war dabei die obere Deckschicht soweit zu optimieren, dass sie ohne starke Verluste vom Laserlicht durchdrungen werden kann. Das Ziel wurde erreicht, nachdem die Deckschicht von $5 \mathrm{~nm}$ Ta / $3 \mathrm{~nm} \mathrm{Ru}$ auf $2 \mathrm{~nm}$ Ta / $2 \mathrm{~nm} \mathrm{Ru}$ reduziert wurde. Bei Proben mit deutlich höheren Ta- und Ru-Schichtdicken war der Unterschied zwischen P- und AP-Zuständen nicht erkennbar. Außerdem erwies sich der Austausch der CoFeB-Schichten als ungünstig (dickere Schicht nach unten). In diesem Fall wurde der AP-Zustand ebenfalls nicht ausreichend definiert.

Als erste Charakterisierung der Proben wurden Messungen des Magneto-Optischen-Kerr-Effekts gemacht. Diese Messungen wurden in Abhängigkeit von der CoFeB-Schichtdicke durchgeführt. Der wesentliche Unterschied zu den Messungen aus dem Unterabschnitt 4.1.2 ist die Tatsache, dass es sich jetzt um magnetische Tunnelelemente mit zwei CoFeB-Schichten und nicht um eine CoFeB-Monoschicht handelt. Da die Dicken der CoFeB-Schichten unterschiedlich sind (pseudo-spin-valve 


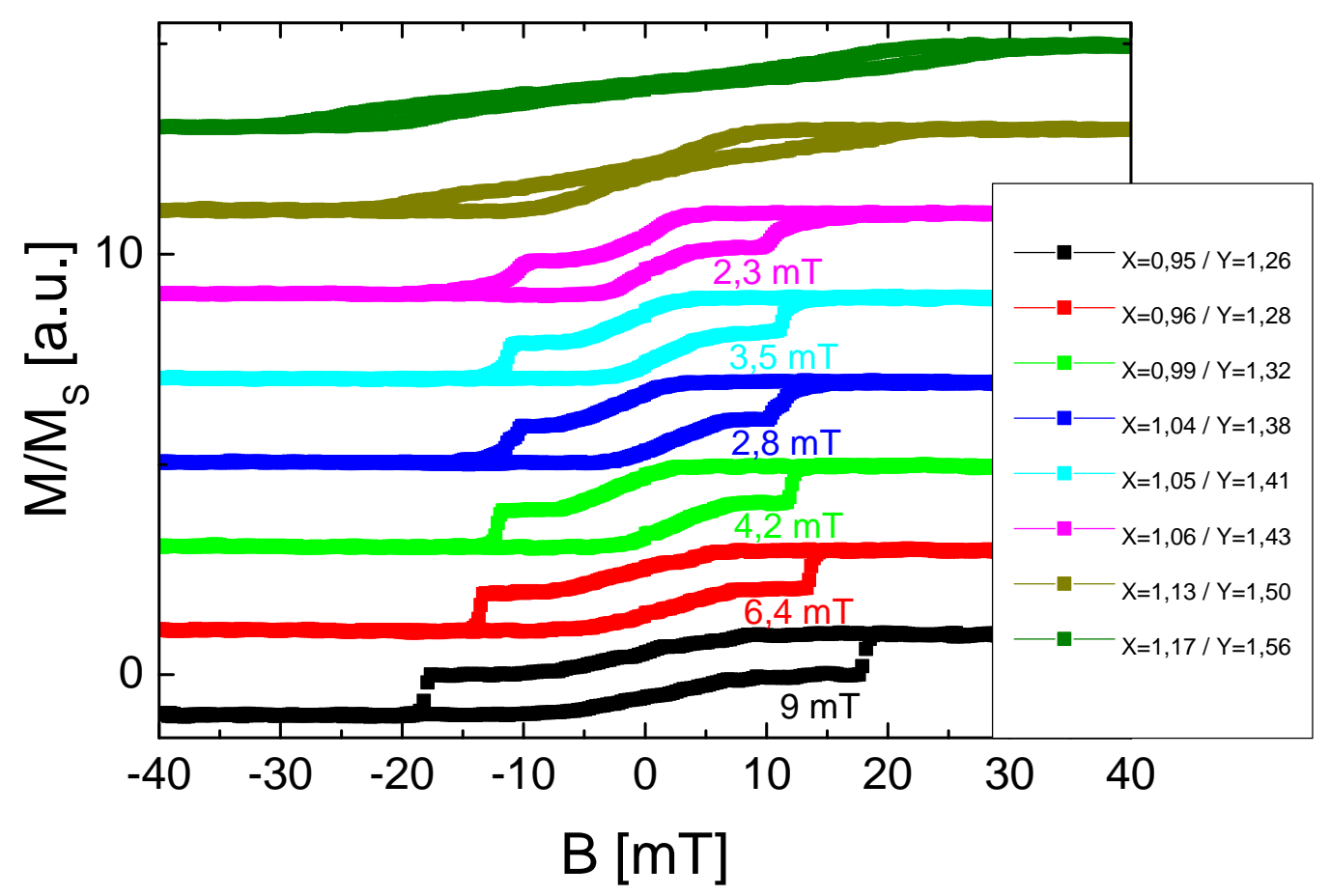

Abbildung 4.21.: Hysterese-Kurven der Probe in Abhängigkeit von der CoFeBDicke. Die ferromagnetische Schichten mit einer Barriere sind wie folgt aufgebaut: X nm CoFeB / 1,26 nm MgO / Y nm CoFeB. X und Y bezeichnen die entsprechenden Dicken der CoFeB-Schichten. Farblich unterschiedliche Feldangaben beschreiben die Plateau-Breite des AP-Zustandes.

system), kann durch die Magnetfeldwahl analog zu dem TMR-Effekt ein antiparalleler Zustand eingestellt werden. Die entsprechende Magnetfeldeinstellung wird in dem nächsten Unterabschnitt relevant, wo die Demagnetisierung der Probe im parallelen und antiparallelen Zuständen untersucht wird.

Die Abbildung 4.21 zeigt, dass das magnetische Verhalten der Probe sich mit den steigenden Schichtdicken der Ferromagneten deutlich ändert. Insbesondere werden mit steigender CoFeB-Schichtdicke die Plateaus, die dem AP-Zustand entsprechen, schmaler. Dies hat zur Folge, dass der AP-Zustand immer schwächer definiert wird, bis der Unterschied zwischen P- und AP-Zuständen bei einer CoFeB-Schichtdicke von etwa 1,5 nm komplett unidentifizierbar wird. Das Verhalten der Hysteresen für die CoFeB-Schichtdicken ab 1,5 nm entspricht dem Verhalten des Systems in dem magnetischen Grundzustand, welches durch Streifendomänen repräsentiert wird. 


\subsubsection{Magnetisches Verhalten der MTJs auf ultrakurzen Zeitskalen}

An der Probe aus dem Unterabschnitt 4.3.2 wurden Messungen auf ultrakurzen Zeitskalen gemacht. Dafür wurde ein All-Optical Pump-Probe Experiment mit einem fs-Lasersystem verwendet. Das Lasersystem liefert Pulse mit einer zeitlichen Pulslänge von ca. 55-60 fs. Die Fluenz der Anregung wurde von $20 \frac{\mathrm{mJ}}{\mathrm{cm}^{2}}$ bis $60 \frac{\mathrm{mJ}}{\mathrm{cm}^{2}}$ bei konstant gehaltenem Strahldurchmesser auf der Probe variiert. Das angelegte Magnetfeld hatte eine out-of-plane Ausrichtung und konnte bei Bedarf für einen Wert konstant gehalten werden. Für die Einstellung des parallelen Zustands der Ferromagneten wurde ein ausreichend starkes Magnetfeld (Sättigungsfeld der Magnetisierung) angelegt. In der Regel lag der Wert bei $B_{P}= \pm 30 \mathrm{mT}$. Die Magnetfeldwerte für die antiparallele Ausrichtung wurden aus den Daten in Abbildung 4.21 ermittelt und entsprachen den Plateaus in den Hysteresekurven. Je nach Position auf der Probe lagen sie im Bereich von $\pm[8 \mathrm{mT}, 18 \mathrm{mT}]$. Bei der Vermessung der Pund AP-Zustände wurden die externen Magnetfelder in folgender Reihenfolge eingestellt: $-B_{P} \rightarrow B_{A P} \rightarrow B_{P} \rightarrow-B_{A P}$. So wurde gewährleistet, dass beim Übergang von der parallelen zur antiparallelen Ausrichtung die dickere FM-Schicht ihre Magnetisierung beibehält und die dünnere FM-Schicht ihre Magnetisierung umschaltet.

In Abbildung 4.22 sind Messungen der Demagnetisierung an der gleichen Position (hier Position C) mit unterschiedlichen Fluenzen abgebildet. Mit steigender Fluenz erhöht sich erwartungsgemäß die gemessene Amplitude der Entmagnetisierungskurven. In beiden magnetischen Zuständen findet eine schnelle Entmagnetisierung der Schichten auf Zeitskalen von etwa 80-170 fs statt (siehe Tabelle 4.7). Am Verhalten der Entmagnetisierungskurven (insbesondere bei der Entmagnetisierungsdauer, siehe Tabelle 4.7) lässt sich ein Unterschied zwischen den P- und AP-Zuständen, welcher auch bei den Messungen von W. He et al. [40] erkennbar ist, für hohe Fluenzen feststellen. Bei niedrigeren Fluenzen weist der P-Zustand eine schnellere Demagnetisierung als der AP-Zustand auf. Das Verhalten kehrt sich bei höheren Fluenzen um, so dass die Demagnetisierung im AP-Zustand schneller verläuft. Nach der schnellen Phase der Entmagnetisierung tritt eine langsame Phase der Thermalisierung ein. Die Thermalisierung tritt in den in dieser Arbeit verwendeten Proben und in von W. He et al. untersuchten Proben auf Zeitskalen bis zu 1 ns auf.

Eine weitere Messung der Demagnetisierungskurven wurde in Abhängigkeit von den CoFeB-Schichtdicken gemacht. Dabei wurde die Probe in Mikrometerschritten relativ zum Laserstrahl entlang des Schichtdickengradienten verfahren und die entsprechende Positionen wurden notiert. Aus den vermessenen Positionen wurden die Schichtdicken der CoFeB-Schicht berechnet. Die entsprechenden Demagnetisierungskurven sind in Abbildung 4.24 abgebildet. 


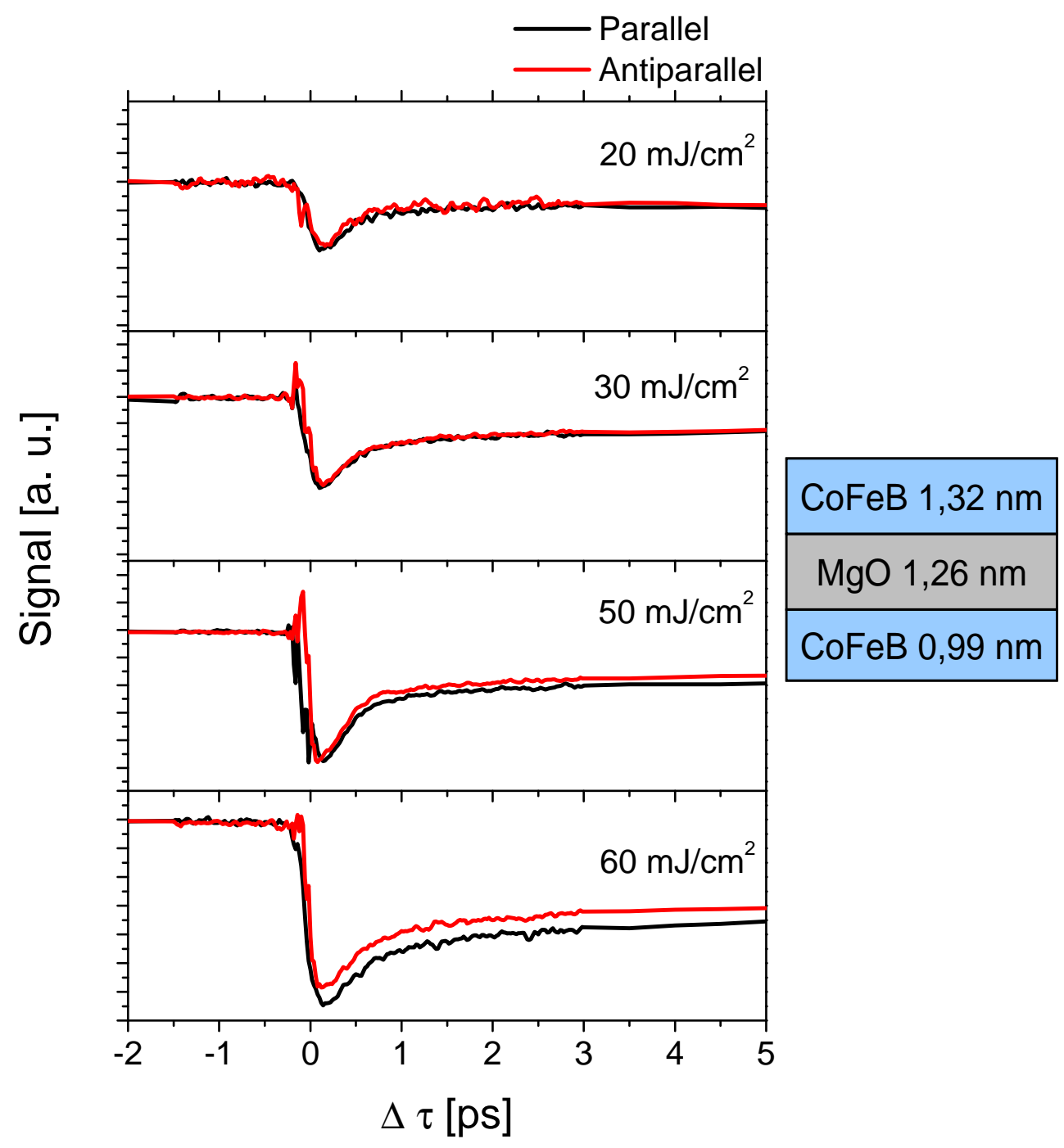

Abbildung 4.22.: Fluenzabhängigkeit der Demagnetisierungskurven. Position C auf der Probe. Die Unterschiede in der Entmagnetisierungsamplitude der P- und AP-Zustände sind erst bei höheren Fluenzen ausgeprägt.

\begin{tabular}{|l|c|c|}
\hline & P & AP \\
\hline \hline Fluenz: $20 \frac{\mathrm{mJ}}{\mathrm{cm}^{2}}$ & $\sim 100 \mathrm{fs}$ & $\sim 165 \mathrm{fs}$ \\
\hline Fluenz: $30 \frac{\mathrm{mJ}}{\mathrm{cm}^{2}}$ & $\sim 110 \mathrm{fs}$ & $\sim 140 \mathrm{fs}$ \\
\hline Fluenz: $50 \frac{\mathrm{mJ}}{\mathrm{cm}^{2}}$ & $\sim 150 \mathrm{fs}$ & $\sim 80 \mathrm{fs}$ \\
\hline Fluenz: $60 \frac{\mathrm{mJ}}{\mathrm{cm}^{2}}$ & $\sim 150 \mathrm{fs}$ & $\sim 110 \mathrm{fs}$ \\
\hline
\end{tabular}

Tabelle 4.7.: Dauer der Demagnetisierung $\tau$ für die Position $\mathrm{C}$ auf der Probe in Abhängigkeit von der Fluenz. 


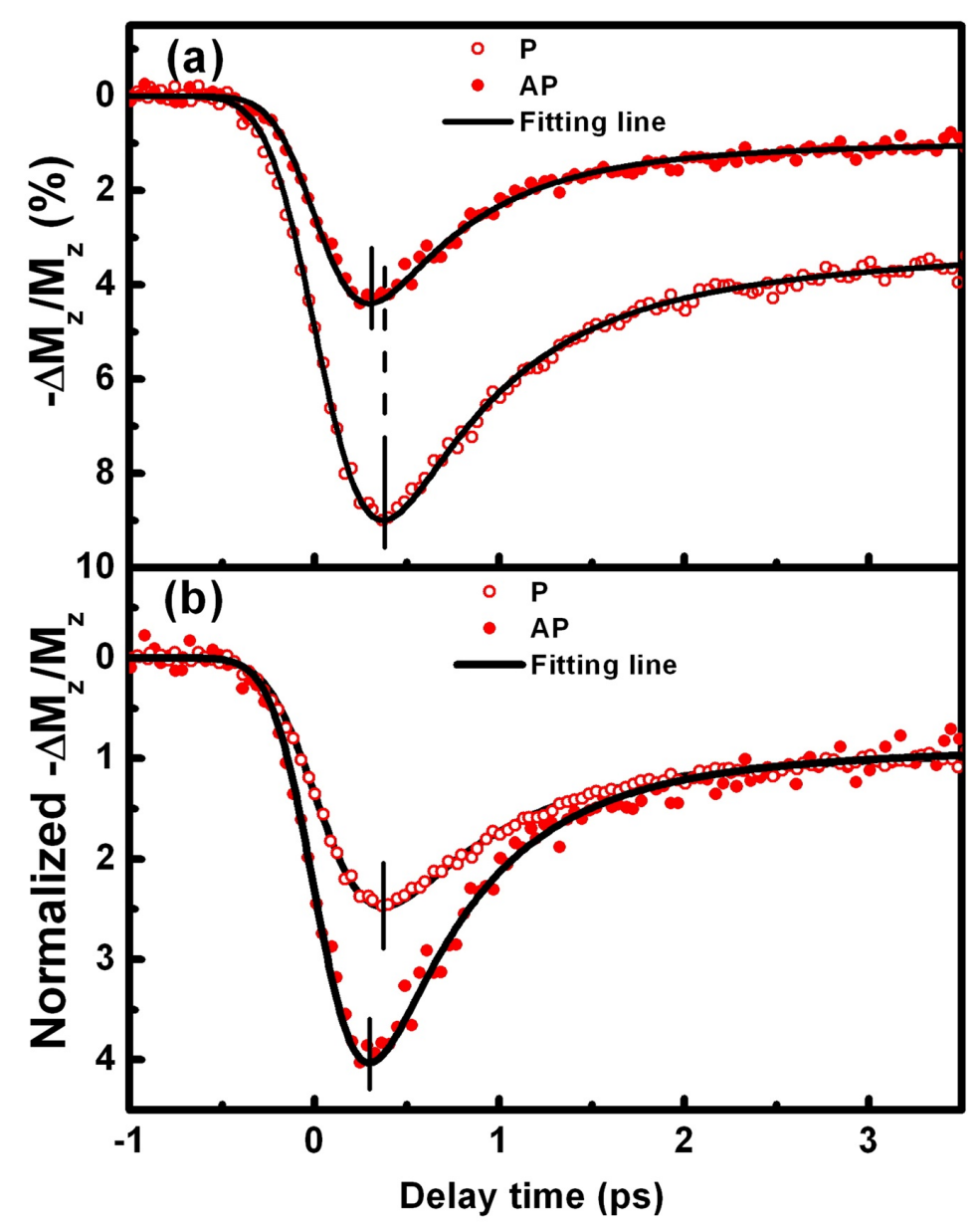

Abbildung 4.23.: Ergebnisse der Dynamikmessungen an MTJs von W. He et al. [40].

In Abbildung 4.24 lässt sich bereits für Positionen mit dünneren CoFeB-Schichten erkennen, dass sich die meisten Entmagnetisierungskurven für die parallele und antiparallele Ausrichtungen unterscheiden: Bis auf die oberste Messung (Position A) zeigen übrige Messungen einen Unterschied zwischen P- und AP-Zuständen. Mit steigender Schichtdicke wird auch der Unterschied größer. Bereits ab der Co$\mathrm{FeB}(1,28) / \mathrm{MgO}(1,26) / \mathrm{CoFeB}(0,96)$ Schichtdicke (Position B) lässt sich eine deutliche Ähnlichkeit des magnetischen Verhaltens mit den Ergebnissen von W. He et al. [40] erkennen. Die Minima der Demagnetisierungskurven sind nach den Zeiten $\tau$ aufgetreten, die in Tabelle 4.8 aufgelistet sind. 


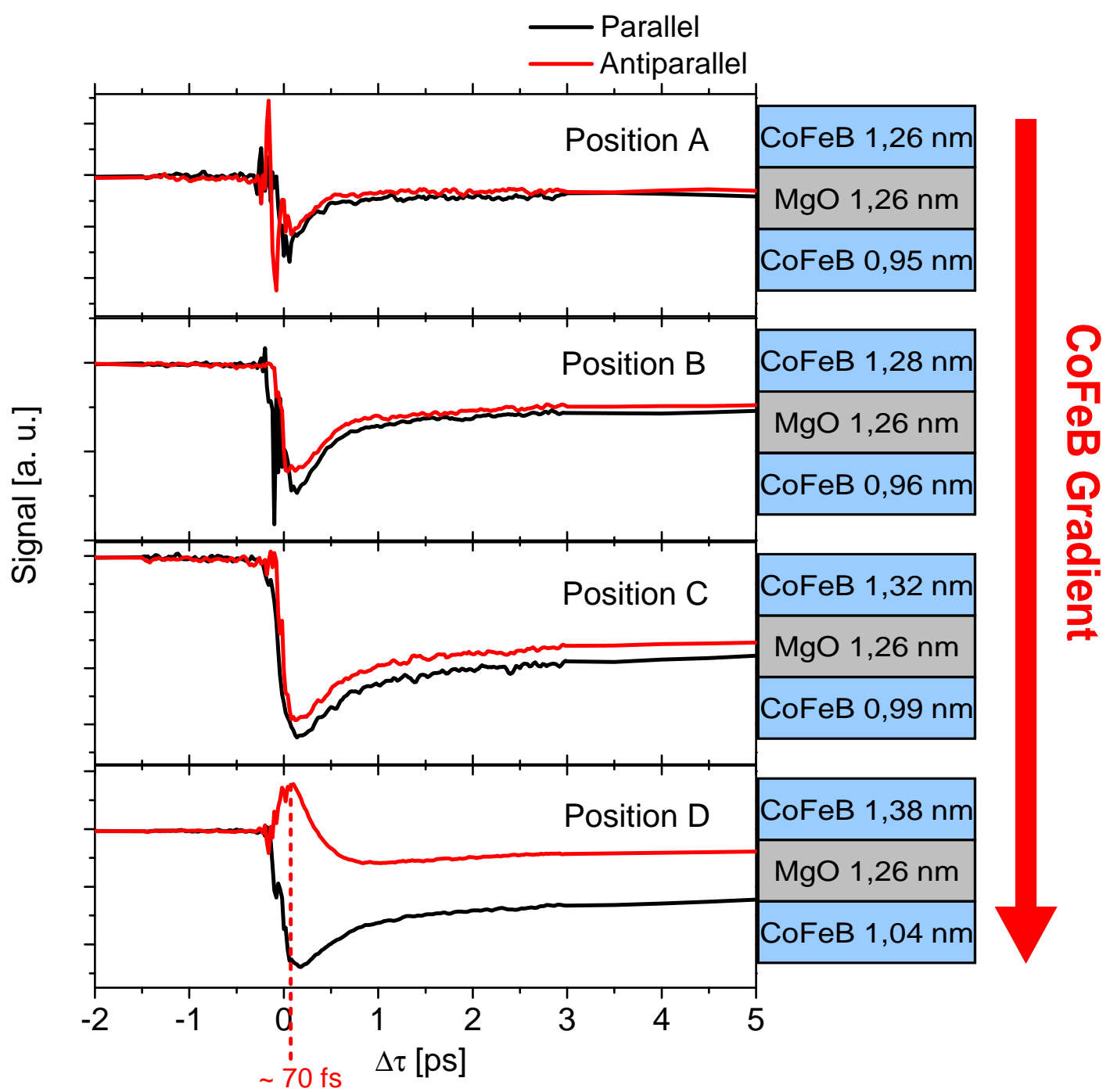

Abbildung 4.24.: Demagnetisierungskurven gemessen an der Probe mit dem CoFeB-Gradienten.

\begin{tabular}{|c|c|c|}
\hline & $\mathrm{P}$ & AP \\
\hline \hline $\mathrm{A}: \mathrm{X}=0,95 / \mathrm{Y}=1,26$ & $\sim 50 \mathrm{fs}$ & $\sim 50 \mathrm{fs}$ \\
\hline $\mathrm{B}: \mathrm{X}=0,96 / \mathrm{Y}=1,28$ & $\sim 135 \mathrm{fs}$ & $\sim 75 \mathrm{fs}$ \\
\hline $\mathrm{C}: \mathrm{X}=0,99 / \mathrm{Y}=1,32$ & $\sim 150 \mathrm{fs}$ & $\sim 110 \mathrm{fs}$ \\
\hline $\mathrm{D}: \mathrm{X}=1,04 / \mathrm{Y}=1,38$ & $\sim 160 \mathrm{fs}$ & $\sim 70 \mathrm{fs}$ \\
\hline
\end{tabular}

Tabelle 4.8.: Dauer der Demagnetisierung $\tau$ in Abhängigkeit von der Position auf der Probe. 


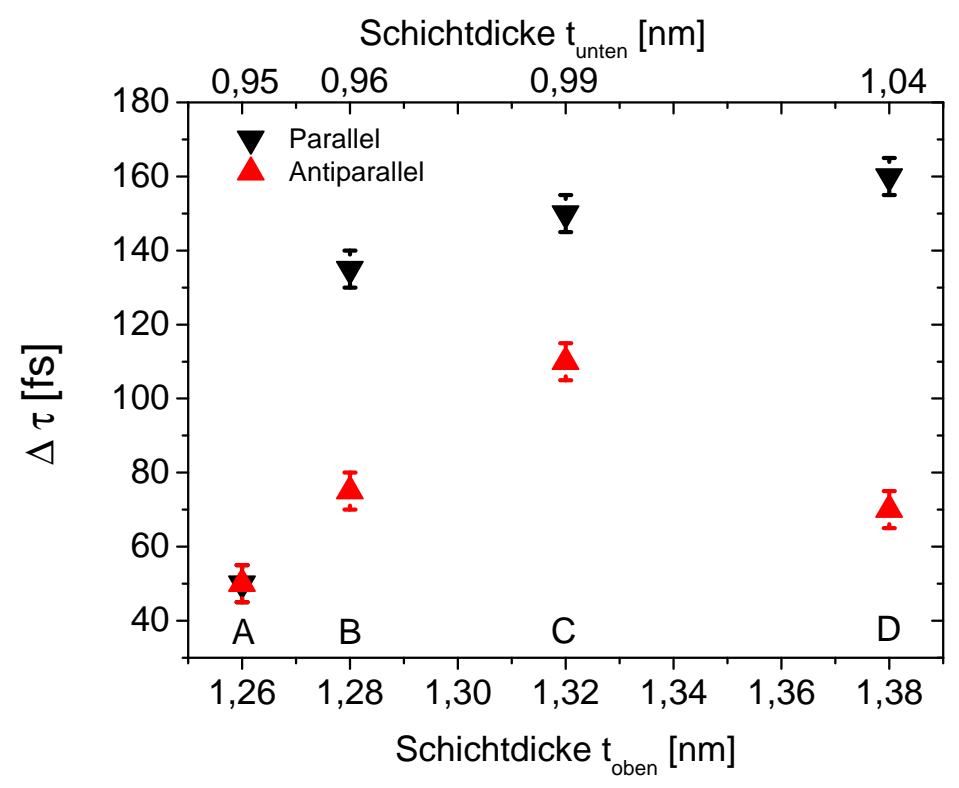

Abbildung 4.25.: Entmagnetisierungsdauer der Positionen A, B, C und D. Für die Position D im AP-Zustand wurde die Magnetisierungsdauer aufgetragen.

Betrachtet man die Demagnetisierungsdauer, so zeigen sich für die Position B [CoFeB $(0,96 / 1,28)]$, C $[\mathrm{CoFeB}(0,99 / 1,32)]$ und D $[\mathrm{CoFeB}(1,04 / 1,38)]$ deutliche Unterschiede zwischen den AP- und P-Zuständen (siehe Abbildung 4.25). Der antiparallele Zustand der Position B entmagnetisiert fast doppelt so schnell wie der parallele. Die Stärke der Entmagnetisierung im AP-Zustand liegt für die Positionen A, B und C im Vergleich zur parallelen Ausrichtung niedriger. Das Verhalten ist vergleichbar mit den Ergebnissen aus [40] (siehe Abbildung 4.23 (a)). Normiert man die Kurven ${ }^{11}$ für Positionen A, B, C analog zur Abbildung 4.23 (b), so liegen die Unterschiede der Entmagnetisierungsstärken für die AP- und P-Zustände bei etwa $5 \%$ (Position A), $12 \%$ (Position B) und 6,5\% (Position C). Durchschnittlich entspricht es etwa 7-8 \%. Dieses Ergebnis liegt niedriger als das von W. He et al. [40] (siehe Abbildung 4.23 (a)). W. He et al. berichteten von ca. $44 \%$ Unterschied zwischen dem AP- und P-Zustand (der parallele Zustand entspricht in diesem Fall den $100 \%)^{12}$.

\footnotetext{
${ }^{11}$ Die normierten Kurven sind in dem Abschnitt A.2 gezeigt.

${ }^{12} \mathrm{~W}$. He et al. haben an dieser Stelle absolute Werte präsentiert. In absoluten Werten entsprach die Absenkung der Amplitudenstärke einem Wert von etwa $4 \%$. Für bessere Vergleichbarkeit wurde in dieser Arbeit die Entmagnetisierungsamplitude der parallelen Ausrichtung als $100 \%$ festgelegt.
} 


\subsubsection{Laser angeregter Spintransport}

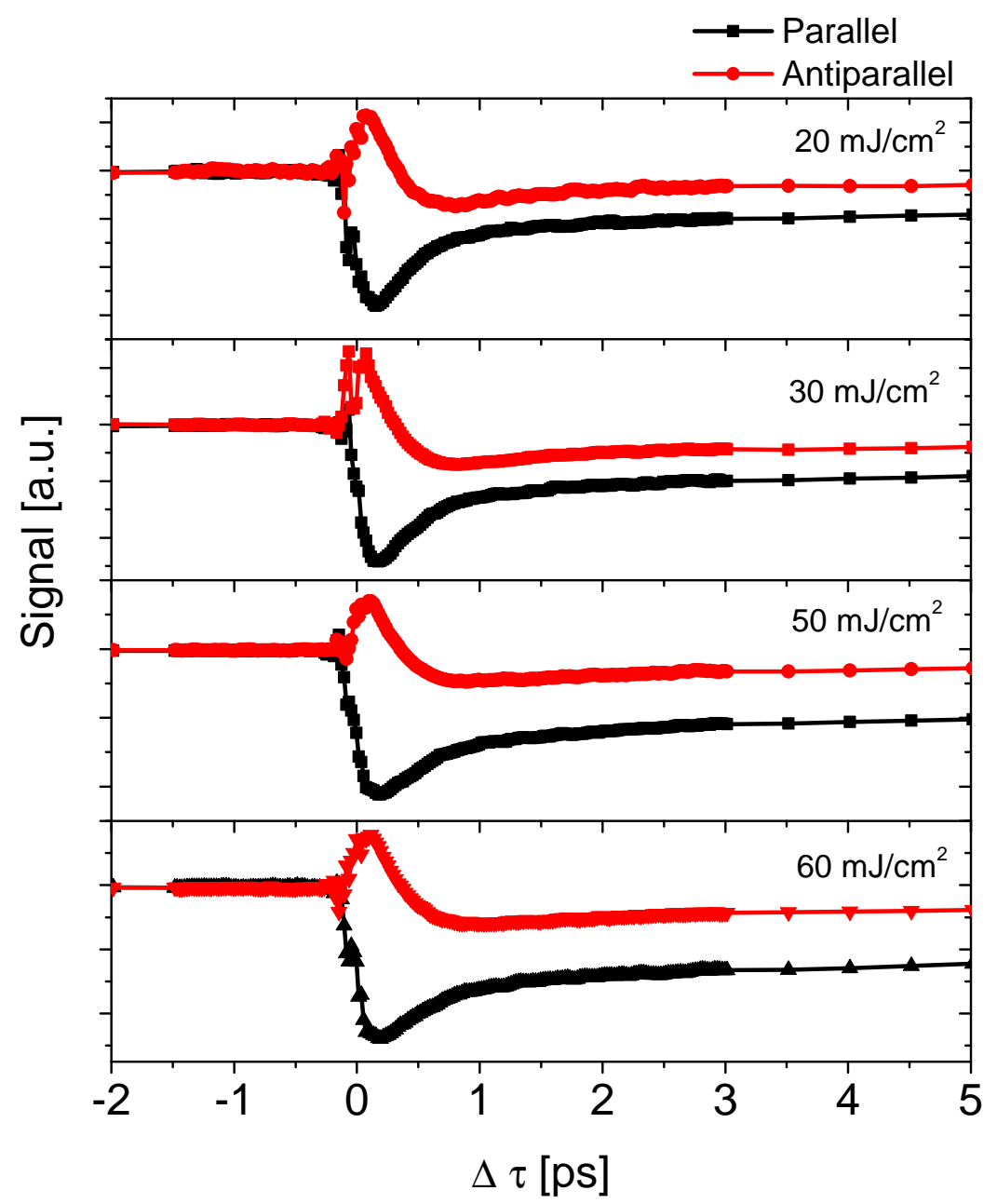

Abbildung 4.26.: Die Fluenzabhängigkeit der Position D mit unterschiedlichem P- und AP-Verhalten der Demagnetisierungskurven. Die Peak-Position für den AP-Zustand liegt (von oben nach unten) bei: 59, 64, 76 und $71 \mathrm{fs.}$

Die Position D zeigt ein von den Positionen A, B und C abweichendes Verhalten. In dem antiparallelen Zustand zeigen die ferromagnetischen Schichten der Position D beim Eintritt eines Laserpulses sogar eine Erhöhung der Gesamtmagnetisierung der Schichten in die out-of-plane Richtung. Über solches Verhalten von Entmagnetisierungskurven wurde von W. He et al. nicht berichtet. Die Dauer der Magnetisierungserhöhung beträgt ca. 70 fs. Danach entmagnetisiert die Probe bis ca. 700 fs, wonach eine lange Phase der Relaxation in den Ausgangszustand folgt. Dieses Verhalten wurde für mehrere Pumplluenzen gemessen (siehe Abbildung 4.26): Das 
Verhalten der Kurven blieb auch für niedrigere Fluenzen $\left(\sim 20 \frac{\mathrm{mJ}}{\mathrm{cm}^{2}}\right)$ erhalten.

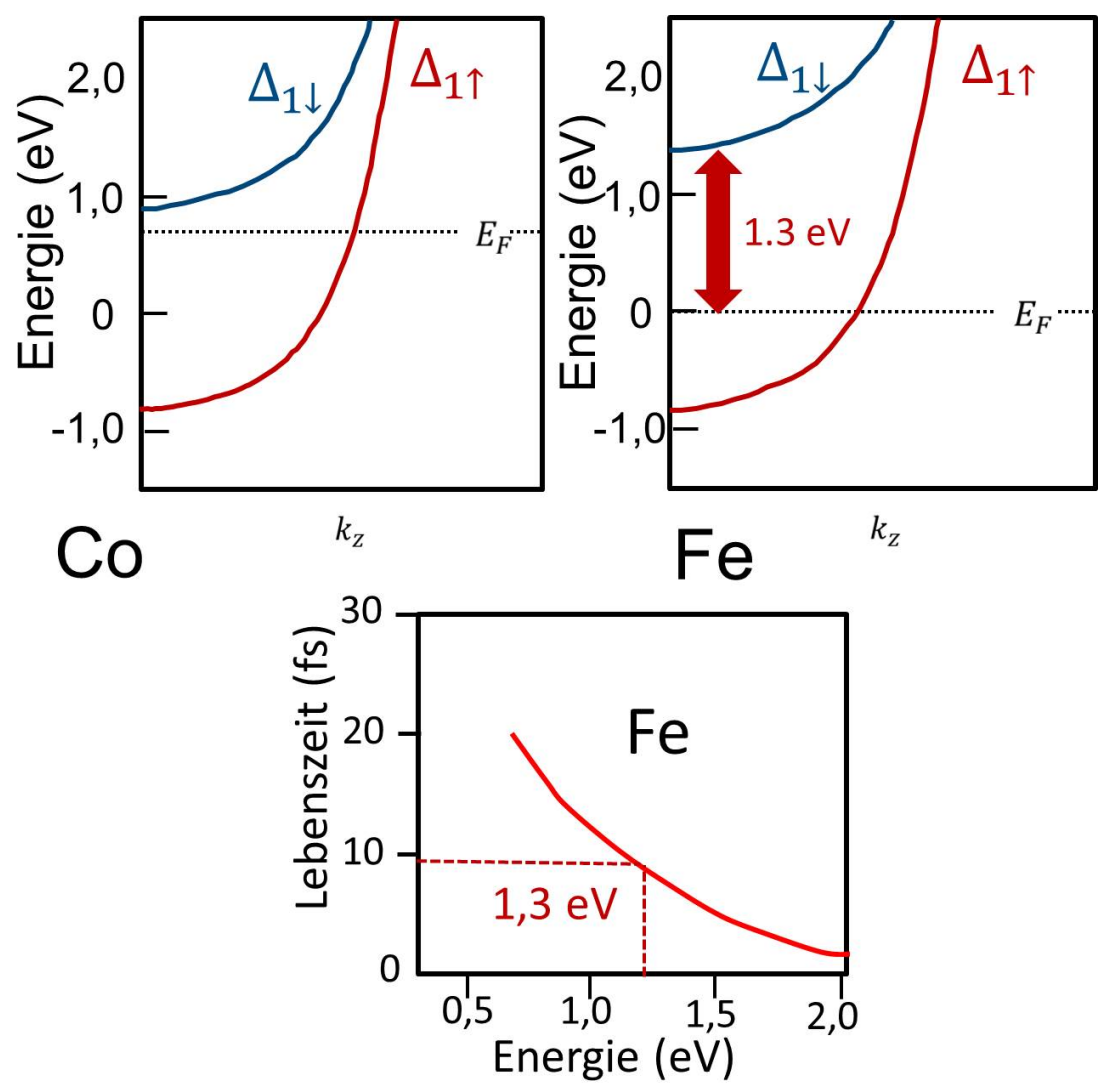

Abbildung 4.27.: Bandschema des $\triangle_{1}$-Energieniveaus für Kobalt und Eisen (nach [20]). Wird eine CoFe-Legierung (wie es bei hier verwendeten Proben der Fall ist) genutzt, so befindet sich das Fermi-Niveau zwischen denen von Co und Fe.

Die nötige Übergangsenergie in der CoFeB-Legierung wird also einen Wert $\leq$ 1,3 eV aufweisen. Die für diesen Wert entsprechende Lebenszeit der angeregten Elektronen in Fe ist nach [39] unten abgebildet. In der CoFeB-Legierung wird es dem Wert für die Lebenszeit der Elektronen von $\geq 10$ fs entsprechen, da die Energie der angeregten Elektronen $\leq 1,3 \mathrm{eV}$ ist.

Geht man von einer Laserpulsdauer von etwa 55-60 fs aus, so erhöht sich die Magnetisierung der Probe an der Position D sogar nach Abklingen des Pulses weiterhin ca. 10-15 fs lang. Dieses Verhalten spricht für durch Laserpulse induzierte Spinströme, die die Probenmagnetisierung erhöhen könnten. Im Folgenden wird diese Möglichkeit genauer untersucht.

In dem Unterabschnitt 4.3.1 wurde bereits das Entstehen der angeregten beweglichen Elektronen in einer Eisenschicht bei einer Wechselwirkung mit einem Laserpuls erwähnt. Die Lebensdauer und Geschwindigkeit der Elektronen weist eine Energieband- und Spinabhängigkeit auf.

Werden die für den Spintransport relevanten Energiebänder betrachtet, so lässt 
sich feststellen, dass aufgrund der Spinfiltereigenschaften der MgO-Barriere die Tunnelwahrscheinlichkeiten für die Elektronen aus den $\triangle_{2}, \triangle_{2^{\prime}}$ und $\triangle_{5^{-}}$Bändern im Vergleich zum $\triangle_{1^{-}}$Band ${ }^{13}$ um mehrere Größenordnungen kleiner sind [24]. Den größten Beitrag zum Spintransport liefern also die Elektronen aus dem $\triangle_{1}$-Band. In Abbildung 4.27 ist das Energieschema für die Majoritätselektronen $\Delta_{1 \uparrow}$ und Minoritätselektronen $\Delta_{1 \downarrow}$ für Co (links) und Fe (rechts) abgebildet. Damit auch die Minoritätselektronen einen Beitrag zum Spintransport leisten können, müssen sie im Falle der Eisenschicht mit einer Energie von mindestens 1,3 eV angeregt werden. So gäbe es für das Tunneln der Minoritätselektronen sogar in der antiparallelen Ausrichtung der MTJs freie Zustände auf der anderen Elektrode. Da in unserem Fall eine CoFeB-Legierung verwendet wird, liegt das Fermi-Niveau zwischen den jeweiligen Niveaus von reinen Co- und Fe-Schichten. Als Folge für $\mathrm{CoFeB}$ ergibt sich für den Minoritätsspintransport eine niedrigere notwendige Anregungsenergie. Aus diesem Grund wird die Anregungsenergie der $\triangle_{1 \downarrow}$-Elektronen als $\leq 1,3 \mathrm{eV}$ angenommen.

V. Zhukov et al. [39] haben bereits die Lebensdauer der angeregten Elektronen in der Eisen-Schicht untersucht. Die Ergebnisse ihrer Experimente sind in Abbildung 4.27 (unten) abgebildet. Der eingezeichnete Pfeil zeigt die Lebenszeit der angeregten Elektronen zu einer Anregungsenergie von etwa 1,3 eV an. Die Lebenszeit beträgt in diesem Fall ca. 10 fs. Für CoFeB mit einer niedrigeren Anregungsenergie ist die Lebenszeit der angeregten Elektronen sogar höher. Wenn man dem Verlauf der Kurve aus Abbildung 4.27 (unten) für kleinere Energien folgt, liegt die Lebenszeit der angeregten CoFe-Elektronen zwischen 10-15 fs.

Die Dauer der Magnetisierungserhöhung bei der Dynamikmessung für Position $\mathrm{D}$ aus Abbildung 4.24 lag bei etwa $\tau_{M a g}=70 \mathrm{fs}$. Mit einer Laserpulsdauer $t_{\mathrm{Puls}}$ von etwa 55-60 fs und einer Lebensdauer der angeregten Elektronen $t_{L}$ von etwa 10-15 fs, ergibt sich folgende Relation:

$$
\tau_{M a g}=t_{\text {Puls }}+t_{L}
$$

Das bedeutet, dass sich die Gesamtmagnetisierung für die Lebensdauer der Elektronen über die Laserpulswirkungsdauer hinaus erhöht. Solche Magnetisierungen der Probe können durch einen Spinstrom zu Stande kommen. Wie bereits erwähnt, kommen die Elektronen aus dem $\triangle_{1}$-Band an der ersten Stelle als Spinleiter in Frage. Wird zusätzlich noch die Tatsache berücksichtigt, dass es nur in dem AP-Zustand zu solch einem Spintransport kommt, kann der Effekt mit Hilfe der Abbildung 4.28 veranschaulicht werden. Abbildung 4.28 (a) zeigt den einfachsten Fall: Parallele Orientierung der Magnetisierung ohne Anregung durch den Pumpstrahl. Die Gesamtmagnetisierung kann als die Summe aller Spins betrachtet werden. Dabei bestimmt die obere ferromagnetische Schicht aufgrund ihrer größeren Dicke die Gesamtmagnetisierung der Probe. Die Beiträge der unteren Schicht können die Gesamtmagnetisierung verstärken oder abschwächen, jedoch die obere Schicht bleibt entscheidend

\footnotetext{
${ }^{13}$ Es handelt sich dabei um die Energiebänder, die in der Nähe des Fermi-Niveaus liegen.
} 
bei der Messung der Magnetisierung. In dem Fall (a) gibt es keine angeregten Elektronen (auch heiße Elektronen genannt), deswegen gibt es auch keine Spinströme zwischen den beiden Elektroden. Im Fall (b) kommt der Pumpstrahl an der Probe an. Die Energie kann von Elektronen absorbiert werden, so dass heiße Elektronen in den ferromagnetischen Schichten entstehen. Da es sich dabei um die parallele Orientierung handelt, können Majoritätselektronen zwischen den Elektroden tunneln. Die Minoritätselektronen können wie bereits erwähnt durch die Energieabsorbtion auf höhere Energiebänder angeregt werden, wodurch sie auch an dem Spintransport teilnehmen können. Ihre Lebenszeit von 10-15 fs reicht aus, um sich zwischen den Elektroden bewegen zu können und somit die Magnetisierung der anderen Elektrode zu beeinflussen. Nach dem schnellen Spintransport ( $\leq 200 \mathrm{fs})$ wird die Energie durch die Thermalisierung auf längeren Zeitskalen ( $\leq 1 \mathrm{~ns}$ ) abgebaut (c).

In dem AP-Zustand ohne den Pumpstrahl (d) entspricht die Gesamtmagnetisierung der Summe aller Spinbeiträge der beiden Ferromagneten: $\lambda_{\text {Ges }}=\lambda_{1, M a j}-$ $\lambda_{2, \text { Maj }}+\lambda_{2, \text { Min }}-\lambda_{1, \text { Min }}$. Wird der Pumpstrahl angeschaltet (e), so wird anders als bei (b) das Tunneln der Majoritätselektronen aufgrund der AP-Orientierung nicht möglich und nur heiße Minoritätselektronen nehmen am Spintransport teil. Dies kann bei günstigen Bedingungen eine Erhöhung der Magnetisierung der oberen Elektrode zur Folge haben, wodurch sich die gemessene Gesamtmagnetisierung erhöhen wird. Damit dieser Effekt stark ausgeprägt ist, muss die Fluenz des Lasers relativ hoch gewählt werden (ab etwa $50 \frac{\mathrm{mJ}}{\mathrm{cm}^{2}}$ ). Hohe Fluenz sorgt für eine hohe Anzahl an angeregten Minoritätselektronen, die am Spintransport teilnehmen können. Nach dem schnellen Spintransport kommt es analog zu (c) zu der Thermalisierung der Schichten.

Für unterschiedliche Positionen an der Probe zeigte der Schritt (e) unterschiedliches Verhalten: bei den Positionen B-C kam es im Vergleich zum parallelen Zustand zu einer abgeschwächten Entmagnetisierung der Schichten. Dieses Ergebnis entspricht den Ergebnissen von W. He at al. [40]. Bei der Position D zeigte die Probe beim Schritt (e) eine Erhöhung der Gesamtmagnetisierung. Die Ursache dieses Verhaltens liegt an einem erhöhten Minoritätsspintransport, der die Magnetisierung der oberen Schicht zur Folge hat. Warum es erst bei den Schichtdicken der Position D auftritt, kann bis jetzt noch nicht eindeutig erklärt werden. Es ist möglich, dass sich mit steigender CoFeB-Schichtdicke auch die Barriereneigenschaften (wie z. B. Glätte) leicht ändern. Dies kann auf die Spintransporteigenschaften einen direkten Einfluss haben. Als eine weitere Vermutung für die Ursache dieses Effekts ist der Einfluss der Anisotropieeigenschaften der Probe, denn, wie bereits in dem Unterabschnitt 2.5.2 erwähnt, kann sich der Strom eines Tunnelelements je nach dem ob in- oder out-of-plane Anisotropie vorliegt um den Faktor $\frac{2 \pi M_{e f f}}{H_{k}}$ unterscheiden. Da es sich jedoch bei dieser Probe nicht um einen Tunnelelement handelt, wird der Einfluss der Anisotropie auf den Strom als deutlich geringer eingeschätzt. 


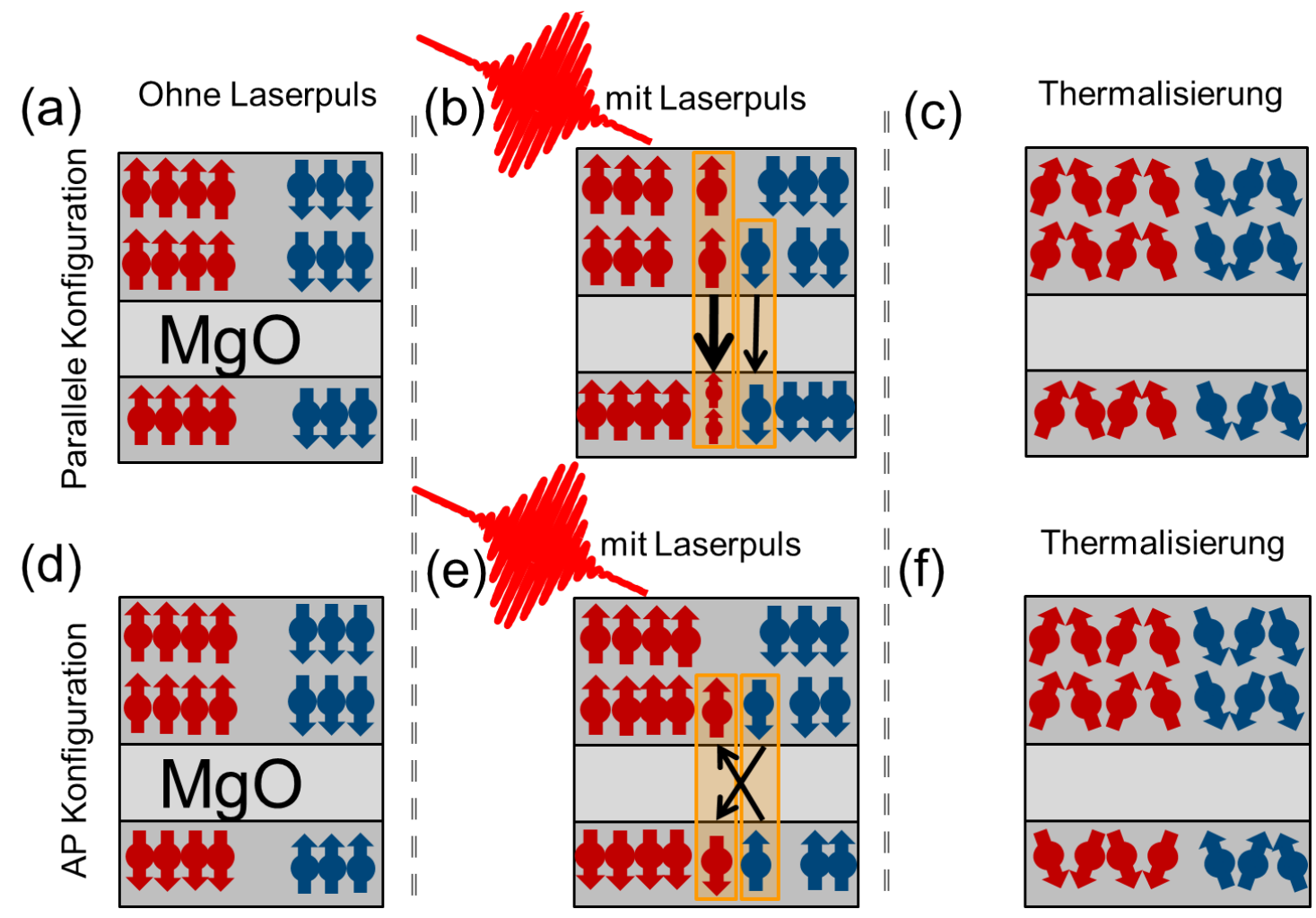

Abbildung 4.28.: Spintransportmodell für Position D. Rote Pfeile entsprechen den Majoritätsspins, blaue - den Minoritätsspins. Die beiden CoFeB-Elektroden sind unterschiedlich dick: die obere Elektrode ist eine CoFeB-Monoschicht dicker und besitzt somit mehr potenzielle Ladungs- und Spinträger. Im Fall der parallelen Ausrichtung der Magnetisierung ( $\mathrm{a}, \mathrm{b}$ und $\mathrm{c}$ ) entstehen unter Einwirkung des Laserstrahls (mit ausreichender Leistung) zwei Spintransport-Kanäle (b): Majoritätskanal und Minoritätskanal. Aufgrund der höheren Anzahl der Zustände werden über den Majoritätskanal mehr Spins transportiert. Nach dem schnellen Spintransport kommt es zur Thermalisierung der Schichten (c). Im AP-Fall (d, e und f) können sich unter Anregung des Laserstrahls die Majoritätelektronen am Spintransport nicht mehr beteiligen. Durch die Laseranregung können jedoch die Minoritätselektronen an dem Transport teilnehmen. Anzahl der tunnelnden Minoritätselektronen muss nicht für beide Elektroden zwangsläufig gleich sein. Ist die Fluenz nicht ausreichend, um die Minoritätselektronen in den Leitungsband anzuregen, so kann der Minoritätskanal nicht geöffnet werden und der Transport der Minoritätselektronen nur durch die Spin-flips stattfindet. 


\section{Zusammenfassung und Ausblick}

\subsection{Magnetische Anisotropie der CoFeB-Schicht}

Im Rahmen dieser Arbeit wurden die Eigenschaften von magnetischen Tunnelelementen mit senkrechter magnetischer Anisotropie (kurz PMA) untersucht. Zu diesem Zweck wurden zunächst für die senkrechte Anisotropie essentielle Parameter ermittelt und für die Herstellung magnetischer Tunnelelemente optimiert. Insbesondere wurde die kritische CoFeB-Schichtdicke $t \sim 1,35 \mathrm{~nm}$ bestimmt, bei der der Übergang von der out-of-plane zur in-plane Anisotropie stattfindet. Dies ermöglichte eine kontrollierte Manipulation der magnetischen Anisotropie in dünnen $\mathrm{CoFeB} / \mathrm{MgO} / \mathrm{CoFeB}$-basierten Tunnelelementen. Der in der Literatur abweichende Wert für die kritische CoFeB-Schichtdicke ( 1,5 nm [27] und $>1,5 \mathrm{~nm}[25])$ liegt durchschnittlich höher als der von den in dieser Arbeit hergestellten Proben $(\sim 1,35 \mathrm{~nm})$. Eine mögliche Ursache dafür kann eine andere CoFeB-Legierung sein, denn die für diese Arbeit hergestellten Proben haben eine $\mathrm{Co}_{25} \mathrm{Fe}_{55} \mathrm{~B}_{20}$-Legierung, während Ikeda et al. [27] und Lee at al. [25] $\mathrm{Co}_{20} \mathrm{Fe}_{60} \mathrm{~B}_{20}$ benutzt haben. Da insbesondere die Hybridisierung der Eisen- und Sauerstoff-Orbitale die senkrechte Anisotropie verstärkt (siehe Abschnitt 2.3), ist die Eisenkonzentration in der ferromagnetischen Schicht von großer Bedeutung.

Der Einfluss der Tantalschicht auf die Anisotropie ist von aktuellem Forschungsinteresse, da die physikalischen Mechanismen noch nicht vollständig geklärt sind. Als eine mögliche Ursache für die Anisotropiebeeinflussung wurden von C. W. Cheng et al. [57] die durch die Tantalschicht verstärkten Diffusionsvorgänge vorgeschlagen. Im Rahmen einer Kollaboration mit der Arbeitsgruppe von Prof. Dr. Seibt (IV. Physikalisches Institut, Georg-August-Universität Göttingen) wurden an den für diese Arbeit hergestellten Proben EELS-Messungen durchgeführt. Die Spektroskopie zeigte eine von der gewählten Pufferschicht abhängige Bordiffusion [52, 51]. Durch die Eigenschaft Boratome während der Auslagerung aus der CoFeB-Schicht aufnehmen zu können, erwies sich Tantal als ein interessantes Material für die Verwendung als Pufferschicht. In dieser Arbeit wurde Tantal als Pufferschicht im Hinblick auf seine Beeinflussung der Anisotropieeigenschaften der CoFeB-Schicht untersucht. Dafür wurden Tantal- und MgO-Pufferschichten verglichen.

In den magnetischen Tunnelelementen sorgt die $\mathrm{CoFeB} / \mathrm{MgO}-$ Grenzschicht aufgrund der Fe 3d- und $\mathrm{O}$ 2p-Orbital-Hybridisierung für die Erhöhung der senkrechten magnetischen Anisotropie (siehe Abschnitt 2.3). Durch die Verwendung der MgOPufferschicht entsteht ein Schichtstapel mit zwei CoFeB/MgO-Grenzflächen. Die Er- 
wartung in diesem Fall ist: Ein $\mathrm{MgO} / \mathrm{CoFeB} / \mathrm{MgO}$-Schichtstapel zeigt eine höhere PMA als der Schichtstapel mit Tantalpufferschicht, der nur eine CoFeB/MgO-Grenzfläche hat. In diesem Kontext wurden die magnetischen Hysteresen der beiden Proben in Abhängigkeit von der CoFeB-Schichtdicke gemessen.

Die Ergebnisse der Messung haben gezeigt, dass trotz der vorhergesagten höheren Anisotropie bei der Probe mit MgO-Pufferschicht die Probe mit der Ta-Pufferschicht deutlich höhere Werte für senkrechte Anisotropie aufweist. Die Erklärung dafür liegt in der Bordiffusion, welche im Falle der MgO-Pufferschicht nicht aus der CoFeBSchicht in die Tantalschicht verlaufen kann. Dadurch bleibt Bor in der CoFeB-Schicht oder setzt sich sogar an der $\mathrm{CoFe} / \mathrm{MgO}$-Grenzfläche ab. Dies hat eine Störung des senkrechten magnetischen Beitrags der Grenzschicht zur Folge (siehe Abschnitt 2.3 und Unterabschnitt 4.1.3). Durch die Eigenschaft die Bordiffusion und somit auch die magnetische Anisotropie der auf dünnen $\mathrm{CoFeB} / \mathrm{MgO}$-basierten magnetischen Tunnelelemente beeinflussen zu können, zeigt sich Tantal als ein für die senkrechte magnetische Tunnelelemente essentielles Material.

\subsection{Spin-transfer torque}

In Rahmen der Untersuchung des Spin-Transfer-Torques der Tunnelelemente mit senkrechter magnetischer Anisotropie wurde ein passender Schichtstapel mit folgenden Schichten hergestellt: SiOx-Substrat / Ta $15 \mathrm{~nm} / \mathrm{CoFeB}$ 1,1 nm / MgO 0,84 $\mathrm{nm} / \mathrm{CoFeB} 1,32 \mathrm{~nm} / \mathrm{Ta} 5 \mathrm{~nm} / \mathrm{Ru} 3 \mathrm{~nm}$. Nach der Herstellung des Schichtstapels mit senkrechter magnetischer Anisotropie wurden die Proben strukturiert und charakterisiert. Zur Strukturierung wurde die Mehrschrittlithographie verwendet, welche die Herstellung der Tunnelelemente mit Minimaldurchmesser bis $100 \mathrm{~nm}$ ermöglichte. Dafür wurden zusätzlich die zur Kontaktierung benötigten Kontakte in passender Form via Argonätzen ausgeschnitten.

An den strukturierten Proben wurden zur Charakterisierung der TMR-Effekt und Spin-Transfer-Torque (kurz STT) gemessen. Die gemessenen TMR-Werte lagen bei $\sim 250-280 \%$ für die Proben mit in-plane Anisotropie und bei $\sim 40-60 \%$ für die Proben mit senkrechter Anisotropie. Die TMR-Werte der Proben mit PMA sind deutlich niedriger als die TMR-Werte der Proben mit IPA. Der Grund dafür ist jedoch nicht die Anisotropieausrichtung, sondern die dünnere $\mathrm{MgO}$-Barriere, die für die Spin-Transfer-Torque-Messungen von Bedeutung ist. Eine dünnere Barriere verringert den Widerstand der Tunnelelemente und somit auch den kritischen Schaltstrom. Jedoch wird dadurch auch der gemessene TMR-Wert kleiner.

An den Tunnelelementen mit PMA wurde Spin-Transfer-Torque gemessen. Dafür wurden bei jedem Tunnelelement ca. 150-400 mal die Strom-Widerstand-Kennlinie (IR-Kennlinie) vermessen. Aus den relativen Häufigkeitsverteilungen für die Schaltströme wurden die entsprechenden Wahrscheinlichkeitsverteilungen bestimmt. Anschließend wurden die kritischen Ströme mit den Schaltwahrscheinlichkeiten über 95 \% für jeweils AP-P- und P-AP-Übergänge ermittelt (siehe Unterabschnitt 4.2.2). Die Schaltströme der in dieser Arbeit verwendeten Tunnelelemente sind in Tabelle 4.5 
aufgelistet und liegen zwischen etwa $[-9,5 \mu \mathrm{A} ;-5,2 \mu \mathrm{A}]$ für den AP-P-Übergang und zwischen $[27,7 \mu \mathrm{A} ; 41,9 \mu \mathrm{A}]$ für den P-AP-Übergang. Diese Werte liegen ca. Faktor 9 (über alle Werte gemittelt) niedriger als die von Sato et al. [55] an ähnlichen Schichtenstapel gemessene kritische Schaltströme. Da niedrigere Schaltströme kleineren Energieverbrauch bedeuten, kann dies von Vorteil für Anwendungen sein.

In dem Unterabschnitt 2.5.2 wurde ein Parameter eingeführt, der die Stabilität der Tunnelelemente bei ständiger Nutzung über die Zeit beschreibt. Dies ist die sogenannte thermische Stabilität $\triangle$. Wie bereits in dem Unterabschnitt 2.5.2 erklärt wurde, liegen die Qualitätskriterien der für die Anwendungen relevanten Schaltelemente relativ hoch. So wird für ein STT-Element eine Lebensdauer von mindestens 10 Jahren bei einer permanenten Nutzung (Lese-/Schreibvorgänge jede 10 ns) erwartet. Diese Lebensdauer entspricht einer thermischen Stabilität von etwa $\triangle \geq 67$. In diesem Kontext ist die thermische Stabilität der Tunnelelemente neben dem Schaltstrom ein weiteres essentielles Qualitätsmerkmal für spätere Anwendungen. Aus diesem Grund wurde die thermische Stabilität für die in dieser Arbeit präsentierten Tunnelelemente anhand der Messdaten für den kritischen Schaltstrom berechnet (siehe Unterabschnitt 4.2.3).

Der höchste Wert für die thermische Stabilität lag bei $\triangle=15,5$. Dieser Wert ist also etwa Faktor 4,3 kleiner als der optimale für kommerzielle Anwendungen und Faktor 2,9 kleiner als von H. Sato et al. [55] gemessen wurde. Da es sich bei den Proben aus dieser Arbeit um die ersten STT-Messungen an den in der Arbeitsgruppe hergestellten Tunnelelementen handelt, kann dieses Ergebnis nicht unter gleich strengen Qualitätskriterien betrachtet werden wie die für die Anwendungen relevante Elemente.

Insgesamt wurde das Ziel, die magnetischen Tunnelelemente mit möglichst niedrigen Schaltströmen herzustellen, erreicht. Dies geschah jedoch unter einem Verlust an thermischer Stabilität der Tunnelelemente. Die beiden Parameter (kritische Schaltströme und thermische Stabilität) können noch weiter optimiert werden. Um die in dieser Arbeitsgruppe hergestellten Tunnelelemente attraktiver für die Anwendungen zu machen, muss zunächst die thermische Stabilität erhöht werden.

Eine Erhöhung der thermischen Stabilität verspricht die von H. Sato et al. [48, 55] vorgeschlagene Modifikation des Schichtstapels: eine der CoFeB-Schichten des $\mathrm{CoFeB} / \mathrm{MgO} / \mathrm{CoFeB}-S t a p e l s$ wurde mit einer 0,4 nm dicken Tantal-Schicht versehen und es wurde eine zusätzliche MgO-Pufferschicht eingebaut, so dass der neue Schichtstapel folgende Struktur annahm ${ }^{1}$ : / Ta (5) / CoFeB (0.9) / MgO (0.9) / CoFeB (1.6) / Ta (0.4) / CoFeB (1.0)/MgO (0.9). Die Tantal-Schicht kann wie bereits erwähnt Bordiffusion aus der CoFeB-Schicht während der Auslagerung beeinflussen, wodurch die $\mathrm{CoFe} / \mathrm{MgO}-$ Grenzfläche weniger durch Bor gestört wird. Eine weitere $\mathrm{MgO} / \mathrm{CoFeB}-$ Grenzfläche erhöht die senkrechte magnetische Anisotropie (siehe Unterabschnitt 4.1.3), wodurch wiederum die thermische Stabilität erhöht wird: $\triangle=\frac{E_{A} V}{k_{B} T}$, mit $E_{A}$ - die magnetische Anisotropieenergiedichte, $V$ - Volumen der ferromagnetischen Schicht und $\mathrm{T}$ - die Temperatur (siehe auch Unterabschnitt 2.5.2).

\footnotetext{
${ }^{1} \mathrm{Zu}$ einer besseren Übersicht wurden die zusätzlich eingebaute Schichten fett markiert.
} 
Die von H. Sato et al. [55] berichtete Erhöhung der thermischen Stabilität für Proben mit zwei MgO-Schichten und einer mit Tantal verstärkten CoFeB-Schicht lag bei einem Faktor von 1,9. Den Schichtstapel auf diese Weise zu modifizieren, wäre eine interessante Fortsetzung der in dieser Arbeit diskutierten Messreihe und eine Möglichkeit die Parameter der STT-Tunnelelemente im Bezug auf thermische Stabilität zu optimieren. Jedoch soll die Reduktion der kritischen Ströme nicht komplett außer Acht gelassen werden und ein Kompromiss zwischen hoher thermischer Stabilität und niedrigen Schaltströmen wäre für die Anwendungen von Vorteil.

\subsection{Dynamik auf ultrakurzen Zeitskalen}

Einige ausgewählte vollflächig aufgedampfte Proben mit tunnelelementfähigen (jedoch nicht strukturierten) Schichtenstapeln und senkrechter magnetischer Anisotropie wurden auf ultrakurzen Zeitskalen mit Hilfe eines Femtosekundenlasers vermessen. Der verwendete Schichtenstapel war folgendermaßen aufgebaut: SiOx / Ta 15 nm / CoFeB X nm / MgO 1,26 nm / CoFeB Y nm / Ta 2 nm / Ru 2 nm. Die Schichtdicke der CoFeB-Schichten war keilförmig aufgedampft und der Gradient betrug ca. $30 \%$. Der Messstrahl des Lasers wurde entlang des Gradienten verfahren, so dass das magnetische Verhalten für mehrere CoFeB-Schichtdicken untersucht werden konnte. Die zu untersuchende Probenposition wurde mit Hilfe eines Pumpstrahls thermisch angeregt und das magnetische Verhalten wurde zeitlich aufgelöst für die parallele und antiparallele magnetische Orientierung der Probe aufgenommen. Das Hauptziel der Messungen war die Spintransportprozesse in den magnetischen Tunnelelementen genauer zur untersuchen und relevante Abhängigkeiten festzustellen.

Das Entmagnetisierungsverhalten der Probe zeigte für alle CoFeB-Schichtdicken bei höheren Fluenzen des Pumpstrahls Unterschiede zwischen dem parallelen und antiparallelen Zuständen (im Folgenden P- und AP-Zustände). Die antiparallele Orientierung zeigte eine niedrigere Entmagnetisierungsamplitude und Entmagnetisierungsdauer $^{2}$ als die parallele Orientierung (siehe Unterabschnitt 4.3.3). Dieses Verhalten ist vergleichbar mit den Ergebnissen von W. He et al. [40], die eine Absenkung der Entmagnetisierung für den AP-Zustand gezeigt haben. Die in dieser Arbeit untersuchte Proben zeigten Unterschiede in der Entmagnetisierungsamplitude zwischen den P- und AP-Zuständen von durchschnittlich etwa 7-8 \%. Eine Erklärung für das unterschiedliche Verhalten der Entmagnetisierung kann folgendermaßen zusammengefasst werden: die durch den Laserpuls angeregte Elektronen (auch heiße Elektronen genannt) können von einer Elektrode in die andere tunneln. In dem parallelen Zustand können dadurch sowohl die Majoritätselektronen als auch die Minoritätselektronen, die nach Zhukov et al. [39] eine Lebenszeit von ca. 10-15 fs haben und durch die Anregungsenergie auf das Leitungsband angeregt werden, am Spintransport teilnehmen. Im AP-Zustand dagegen wird das Tunneln der Majoritätselektronen, die aufgrund einer hohen Spinpolarisation der CoFe-Legierung

\footnotetext{
${ }^{2}$ Bis auf die Position A, die fast die gleiche Entmagnetisierungsdauer für P- und AP-Zustände
} zeigte. 
keine freie Zustände auf der anderen Elektrode haben, nicht möglich und der Spintransport wird in dem AP-Zustand nur von den Minoritätselektronen dominiert. Diese Erklärung unterscheidet sich leicht von der von W. He et al. [40] vorgeschlagenen durch einen Punkt: W. He et al. berücksichtigen auch den Spintransport durch die Minoritätselektronen, jedoch wird in der Publikation an der Stelle von einem Spin-Flip während des Vorgangs ausgegangen. Die Arbeit von T. Kampfrath et al. [38] zeigte bereits die Möglichkeit eines durch die Minoritätselektronen induzierten Spintransports ohne den Spin-Flip-Effekt (siehe Unterabschnitt 4.3.1). An dieser Stelle kann der Spin-Flip-Effekt beim Spintransport nicht komplett ausgeschlossen werden und er trägt eventuell einen Beitrag zum Spintransport bei, jedoch wird der Beitrag als klein im Vergleich zu den anderen Spintransport-Möglichkeiten angenommen.

Einen weiteren interessanten Effekt zeigte das Entmagnetisierungsverhalten für die Position D der Probe, die den CoFeB-Schichtdicken von $\mathrm{X}=1,04 \mathrm{~nm}$ und $\mathrm{Y}=1,38 \mathrm{~nm}$ entspricht. An der Position D wurde im AP-Zustand statt der Entmagnetisierung eine Magnetisierung der Schichten während der ersten ca. 70 fs seit dem Ankommen des Pumppulses an der Probe gemessen. Da die Magnetisierung der Probe von der oberen (dickeren) CoFeB-Schicht vorgegeben wird, deutet das Ergebnis auf einen Spintransport von der unteren zur oberen Elektrode und die transportierte Spins müssen die Majoritätszustände annehmen (andernfalls wäre eine Abnahme der Magnetisierung beobachtbar). In dem AP-Zustand ist dies beim Transport der Minoritätsspins der Fall, da diese mit den Majoritätselektronen der jeweils anderen Elektrode gleiche Spinausrichtung haben (siehe Abbildung 4.28).

Die hier beschriebenen Effekte laufen auf Zeitskalen von unter 200 fs ab (siehe Tabelle 4.8) und liegen im Bereich der ultraschnellen Dynamik, die für Anwendungen vom Interesse sein kann. Weitere Untersuchungen der Effekte sowie der Gewinn der Kontrolle über den ultraschnellen Spintransport sind von besonderem Interesse. Für die zukünftige Arbeiten kommen ähnliche Experimente an magnetischen Tunnelelementen in Frage. Bei den strukturierten Elementen, bei denen der Spintransport mit einer TMR-Messung vorcharakterisiert werden kann, können weitere Erkenntnisse über die Spintransportprozesse zwischen den ferromagnetischen Schichten gewonnen werden. 



\section{A. Weitere Messungen und Auswertungsschritte}

In diesem Kapitel werden zusätzliche Messungen oder Auswertungsschritte, die in dem Haupttext der Dissertation nicht diskutiert wurden, Vollständigkeit halber gezeigt und gegebenenfalls diskutiert.

\section{A.1. MOKE-Messungen der Probe B}
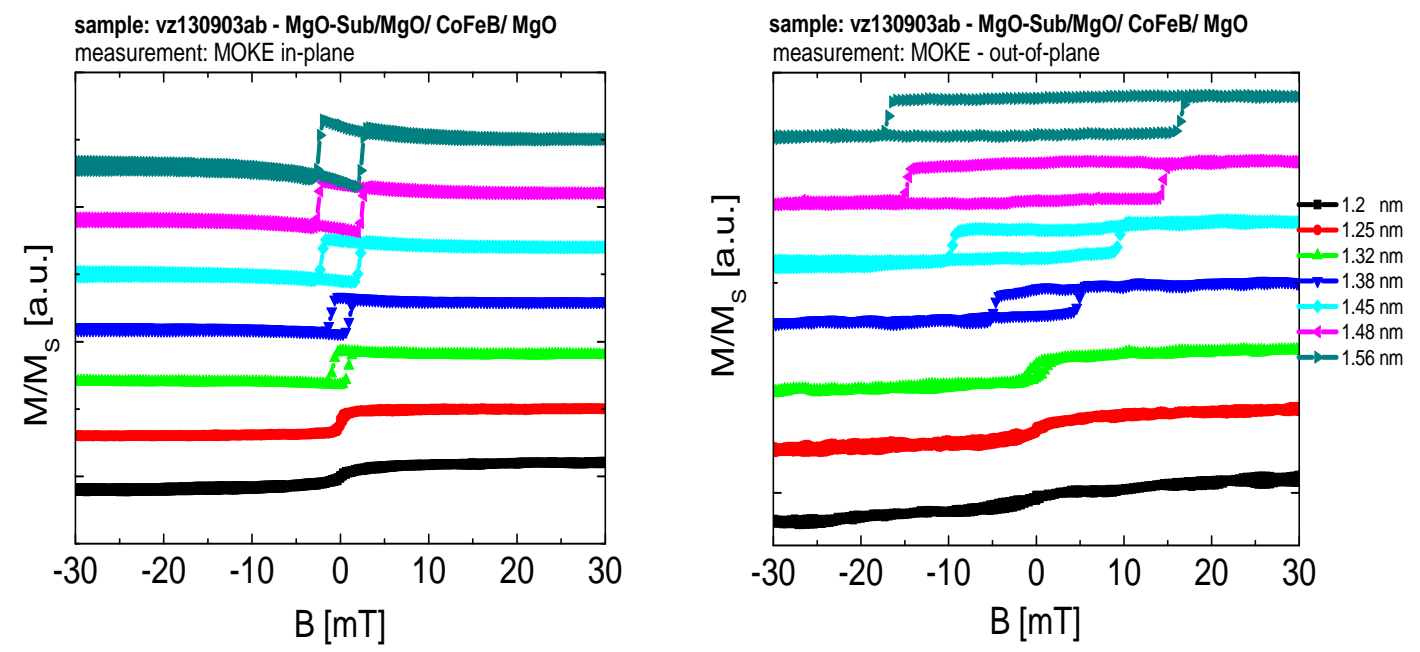

Abbildung A.1.: MOKE-Messungen für alle Schichtdicken der Probe B

Das Verhalten der magnetischen Schicht zeigt keine ausgeprägte leichte Achse. Durch die unkontrollierte Bordiffusion wird die $\mathrm{CoFe} / \mathrm{MgO}-$ Grenzfläche möglicherweise stark mit Bor dotiert. Dies kann die CoFe-Gitteranpassung an $\mathrm{MgO}$ stark stören und zur Abschwächung der Fe- und O-Orbitalhybridisierung führen. 


\section{A.2. Normierung der Dynamikmesskurven}
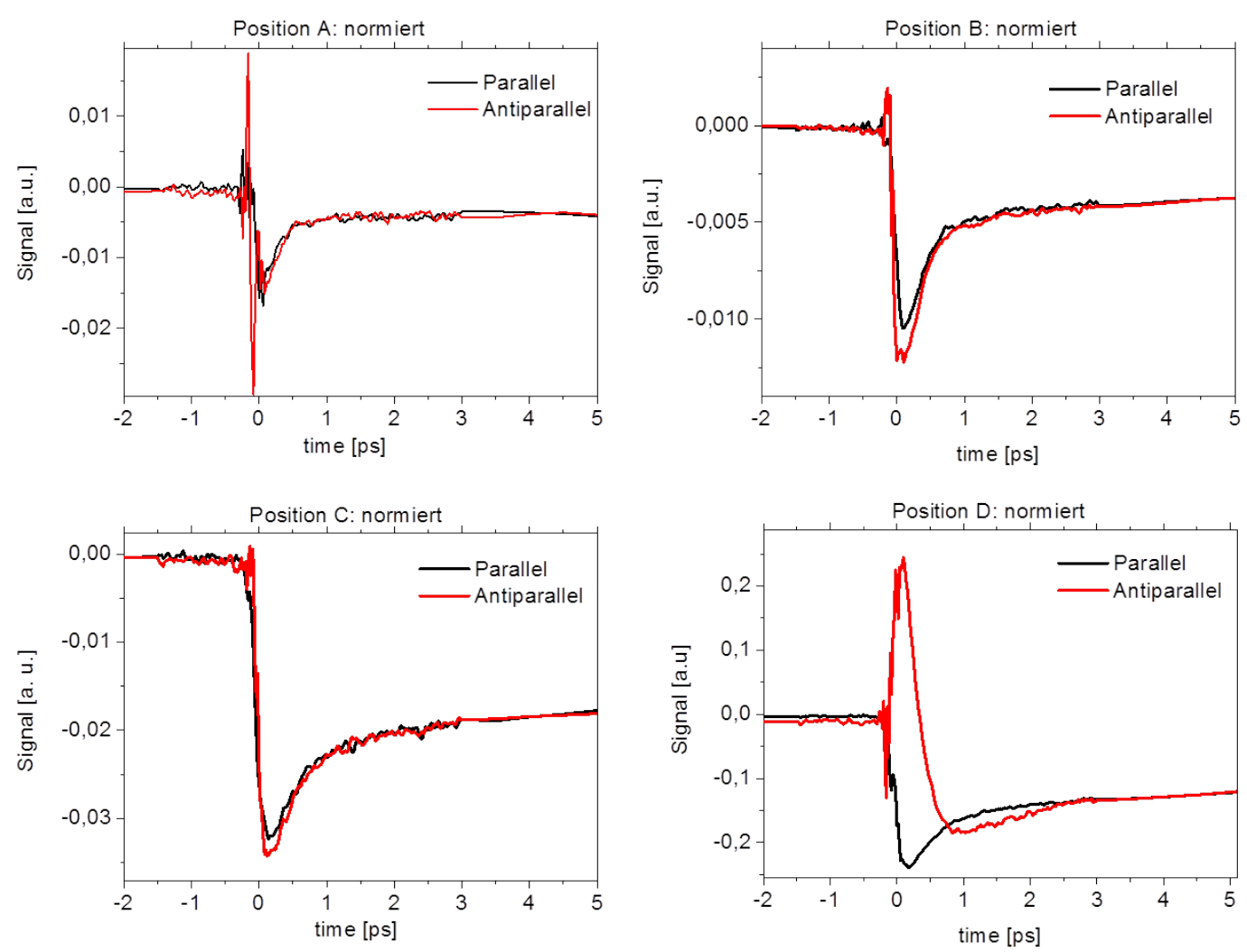

Abbildung A.2.: Normierte Dynamikmessung für die Positionen A, B, C und D an der Probe.

Die Normierung wurde analog zu Abbildung 4.23 (b) gemacht: bei 3 ps wurde der Faktor zwischen P- und AP-Messung bestimmt. Danach wurde die AP-Messung mit diesem Faktor multipliziert. Dies wurde bei Positionen A, B, C und D auf der Probe analog gemacht, um die Vergleichbarkeit mit den Messungen von W. He et al. [40] zu gewährleisten.

\section{A.3. Demagnetisierungsfit für Positionen C und D}

In der Abbildung A.3 sind Ergebnisse eines an den Demagnetisierungskurven gemachten Fits zur Bestimmung der Peak-Position $\tau$ und Breite des Demagnetisierungskurve. Es handelt sich dabei nur um den Demagnetisierungseffekt. Die Magnetisierung am Anfang der Messung der Position D wurde bei dem Fit nicht berücksichtigt.

Bei der Position C scheint sich die Zeit bis zur Demagnetisierung mit einer steigenden Fluenz für kleinere Fluenzen zu erhöhen. Ab etwa $150 \mathrm{~mW}$ der Laserleistung 

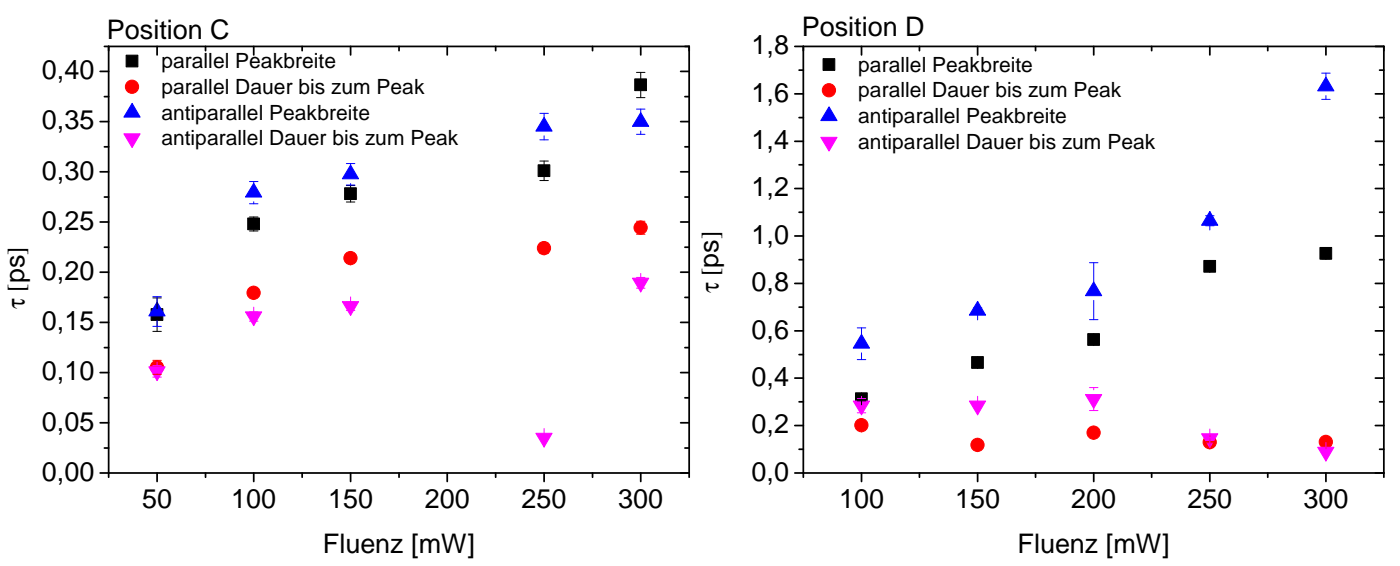

Abbildung A.3.: Ergebnisse eines Fits an den Demagnetisierungskurven. Links befindet sich Position C und rechts Position D.

(entspricht $30 \frac{\mathrm{mJ}}{\mathrm{cm}^{2}}$ ) scheint die Demagnetisierungsdauer den gesättigten Wert erreicht zu haben und erhöht sich nicht mehr. Die Peak-Breite erhöht sich erwartungsgemäß mit steigender Fluenz. Der Ausreißer für AP-Zustand bei $250 \mathrm{~mW}\left(\sim 50 \frac{\mathrm{mJ}}{\mathrm{cm}^{2}}\right)$ kann nicht erklärt werden (auch wenn der Peak ohne den Fit bestimmt wird, liegt der Wert $\tau$ bei etwa $50 \mathrm{fs}$ ), jedoch ist dieser Messpunkt als einziger nicht mit anderen Messungen vergleichbar. An dieser Stelle wird von einer fehlerhaften Messaufnahme ausgegangen.

Position D zeigt mit steigender Fluenz sogar eine leichte Abnahme der Demagnetisierungszeit. Dies kann an der erhöhten Magnetisierung der Schichten durch den Spintransport der Minoritätselektronen liegen (siehe Unterabschnitt 4.3.3). Die Werte für $\tau$ bei der Position D verändern sich mit steigender Fluenz nur gering, wohingegen die Peakbreite einen deutlichen Trend zeigt: mit steigender Fluenz erhöht sich auch die Peakbreite der Demagnetisierungskurven. Dies ist durch eine höhere Demagnetisierung bei höherer Laserleistung erklärbar.

Die Entmagnetisierungszeiten für die Positionen C und D liegen zwischen ca. 100300 fs. Diese Ergebnisse sind vergleichbar mit den Werten von W. He et al. [40] und sind in der Regel unterschiedlich für P- und AP-Zustände. Dies spricht nach [40, 38] für eine auf dem Spintransport basierte Entmagnetisierung, die für Zeiten unter 1 ps erwartet wird. 



\section{Literaturverzeichnis}

[1] D. C. Worledge, M. Gajek, D. W. Abraham, S. Brown, M. C. Gaidis, G. Hu, J. Nowak, E. J. O'Sullivan, R. P. Robertazzi, J. Z. Sun, P. L. Trouilloud, and W. J. Gallagher. Recent Advances in Spin Torque MRAM. In 2012 4th IEEE International Memory Workshop, pages 1-3. IEEE, May 2012.

[2] Yiming Huai. Spin-transfer torque MRAM (STT-MRAM): Challenges and prospects. AAPPS Bulletin, 18(6):33-40, 2008.

[3] P. Khalili Amiri, Z M Zeng, P Upadhyaya, G Rowlands, H Zhao, I N Krivorotov, J.-P. Wang, H W Jiang, J A Katine, J Langer, K Galatsis, and K L Wang. Low Write-Energy Magnetic Tunnel Junctions for High-Speed Spin-Transfer-Torque MRAM. IEEE Electron Device Letters, 32(1):57-59, January 2011.

[4] Xiaochen Guo, Engin Ipek, and Tolga Soyata. Resistive computation. In Proceedings of the 37th annual international symposium on Computer architecture - ISCA '10, page 371, New York, New York, USA, 2010. ACM Press.

[5] M Julliere. Tunneling between ferromagnetic films. Physics Letters A, 54(3):225-226, September 1975.

[6] M. N. Baibich, J. M. Broto, A. Fert, F. Nguyen Van Dau, and F. Petroff. Giant Magnetoresistance of (001)Fe/(001)Cr Magnetic Superlattices. Physical Review Letters, 61(21):2472-2475, November 1988.

[7] G. Binasch, P. Grünberg, F. Saurenbach, and W. Zinn. Enhanced magnetoresistance in layered magnetic structures with antiferromagnetic interlayer exchange. Physical Review B, 39(7):4828-4830, March 1989.

[8] J. S. Moodera, Lisa R. Kinder, Terrilyn M. Wong, and R. Meservey. Large Magnetoresistance at Room Temperature in Ferromagnetic Thin Film Tunnel Junctions. Physical Review Letters, 74(16):3273-3276, April 1995.

[9] T Miyazaki and N Tezuka. Giant magnetic tunneling effect in $\mathrm{Fe} / \mathrm{Al} 2 \mathrm{O} 3 / \mathrm{Fe}$ junction. Journal of Magnetism and Magnetic Materials, 139(3):L231-L234, January 1995.

[10] W. Butler, X.-G. Zhang, T. Schulthess, and J. MacLaren. Spin-dependent tunneling conductance of Fe-MgO-Fe sandwiches. Physical Review B, 63(5):054416, January 2001. 
[11] J. Mathon and A. Umerski. Theory of tunneling magnetoresistance of an epitaxial Fe/MgO/Fe(001) junction. Physical Review B, 63(22):220403, May 2001.

[12] Shinji Yuasa, Taro Nagahama, Akio Fukushima, Yoshishige Suzuki, and Koji Ando. Giant room-temperature magnetoresistance in single-crystal Fe/MgO/Fe magnetic tunnel junctions. Nature materials, 3(12):868-71, December 2004.

[13] S. Ikeda, J. Hayakawa, Y. Ashizawa, Y. M. Lee, K. Miura, H. Hasegawa, M. Tsunoda, F. Matsukura, and H. Ohno. Tunnel magnetoresistance of $604 \%$ at 300 $\mathrm{K}$ by suppression of Ta diffusion in $\mathrm{CoFeB}-\mathrm{MgO}-\mathrm{CoFeB}$ pseudo-spin-valves annealed at high temperature. Applied Physics Letters, 93(8):082508, 2008.

[14] V Khvalkovskiy, D Apalkov, S Watts, R Chepulskii, R S Beach, A Ong, X Tang, A Driskill-Smith, W H Butler, P B Visscher, D Lottis, E Chen, V Nikitin, and M Krounbi. Erratum: Basic principles of STT-MRAM cell operation in memory arrays. Journal of Physics D: Applied Physics, 46(13):139601, April 2013.

[15] Marvin Walter. Untersuchung der Transporteigenschaften von CoFeB-MgOCoFeB- Tunnelmagnetowiderstandselementen. Diplomarbeit, Göttingen. 2009.

[16] Gerrit Eilers. Korrelationen zwischen struktureller Ordnung und elektrischen Transporteigenschaften in $\mathrm{CoFeB}-\mathrm{MgO}-\mathrm{CoFeB}$ Tunnelmagnetowiderstandselementen Dissertation. PhD thesis, Georg-August-University Goettingen, 2009.

[17] Jakob Walowski. Physics of laser heated ferromagnets : Ultrafast demagnetization and magneto-Seebeck effect. PhD thesis, Georg-August-Universität, 2012.

[18] Vladyslav Zbarsky. Charakterisierung und Optimierung der magnetischen Tunnelelemente, Diplomarbeit Göttingen. 2011.

[19] H.C. Stöhr, J., Siegmann. Magnetism From Fundamentals to Nanoscale Dynamics. Springer, Berlin, 2006.

[20] S Yuasa and D D Djayaprawira. Giant tunnel magnetoresistance in magnetic tunnel junctions with a crystalline $\mathrm{MgO}\left(\begin{array}{lll}0 & 0 & 1\end{array}\right)$ barrier. Journal of Physics D: Applied Physics, 40(21):R337-R354, November 2007.

[21] Stuart S P Parkin, Christian Kaiser, Alex Panchula, Philip M Rice, Brian Hughes, Mahesh Samant, and See-Hun Yang. Giant tunnelling magnetoresistance at room temperature with $\mathrm{MgO}$ (100) tunnel barriers. Nature materials, 3(12):862-7, December 2004.

[22] W. F. Brinkman. Tunneling Conductance of Asymmetrical Barriers. Journal of Applied Physics, 41(5):1915, 1970. 
[23] T Fliessbach. Quantenmechanik. Spektrum Akademischer Verlag, Heidelberg, Berlin, 3. edition edition, 2000.

[24] William H Butler. Tunneling magnetoresistance from a symmetry filtering effect. Science and Technology of Advanced Materials, 9(1):014106, April 2008.

[25] Kangho Lee, Jonathan J. Sapan, Seung H. Kang, and Eric E. Fullerton. Perpendicular magnetization of CoFeB on single-crystal MgO. Journal of Applied Physics, 109(12):123910, 2011.

[26] Dongseok Kim, K.Y. Jung, Sungjung Joo, Youngjae Jang, Jinki Hong, B.C. Lee, C.Y. You, J.H. Cho, M.Y. Kim, and K. Rhie. Perpendicular magnetization of $\mathrm{CoFeB}$ on top of an amorphous buffer layer. Journal of Magnetism and Magnetic Materials, 374:350-353, August 2014.

[27] S. Ikeda, K. Miura, H. Yamamoto, K. Mizunuma, H. D. Gan, M. Endo, S. Kanai, J. Hayakawa, F. Matsukura, and H. Ohno. A perpendicular-anisotropy CoFeBMgO magnetic tunnel junction. Nature materials, 9(9):721-4, September 2010.

[28] Vincent Sokalski, Matthew T. Moneck, En Yang, and Jian-Gang Zhu. Optimization of Ta thickness for perpendicular magnetic tunnel junction applications in the MgO-FeCoB-Ta system. Applied Physics Letters, 101(7):072411, August 2012 .

[29] S. Blügel. Magnetische Anisotropie und Magnetostriktion, Magnetische Schichtsysteme, 30. Ferienkurs des Instituts für Festkörperforschung 1999. Institut für Festkörperforschung, Forschungszentrum Jülich GmbH, Jülich, 1999.

[30] H. X. Yang, M. Chshiev, B. Dieny, J. H. Lee, A. Manchon, and K. H. Shin. Firstprinciples investigation of the very large perpendicular magnetic anisotropy at $\mathrm{Fe}-\mathrm{MgO}$ and $\mathrm{Co}-\mathrm{MgO}$ interfaces. Physical Review B, 84(5):054401, August 2011.

[31] C. Tannous and J. Gieraltowski. The Stoner-Wohlfarth model of ferromagnetism. European Journal of Physics, 29(3):475-487, May 2008.

[32] L Berger. Emission of spin waves by a magnetic multilayer traversed by a current. Physical Review B, 54(13):9353-9358, October 1996.

[33] J.C. Slonczewski. Current-driven excitation of magnetic multilayers. Journal of Magnetism and Magnetic Materials, 159(1-2):L1-L7, June 1996.

[34] J. Sun. Spin-current interaction with a monodomain magnetic body: A model study. Physical Review B, 62(1):570-578, July 2000.

[35] E Chen, D Apalkov, Z Diao, A. Driskill-Smith, D Druist, D Lottis, V Nikitin, X Tang, S Watts, S Wang, S A Wolf, A W Ghosh, J W Lu, S J Poon, M Stan, W H Butler, S Gupta, C. K. A. Mewes, Tim Mewes, and P B Visscher. Advances 
and Future Prospects of Spin-Transfer Torque Random Access Memory. IEEE Transactions on Magnetics, 46(6):1873-1878, June 2010.

[36] P. Corkum, F. Brunel, N. Sherman, and T. Srinivasan-Rao. Thermal Response of Metals to Ultrashort-Pulse Laser Excitation. Physical Review Letters, 61(25):2886-2889, December 1988.

[37] A. Eschenlohr, M. Battiato, P. Maldonado, N. Pontius, T. Kachel, K. Holldack, R. Mitzner, A. Föhlisch, P.M. Oppeneer, and C. Stamm. Ultrafast spin transport as key to femtosecond demagnetization. Nature materials, 12(4):332-6, April 2013.

[38] T. Kampfrath, M. Battiato, P. Maldonado, G. Eilers, J. Nötzold, S. Mährlein, V. Zbarsky, F. Freimuth, Y. Mokrousov, S. Blügel, M. Wolf, I. Radu, P. M. Oppeneer, and M. Münzenberg. Terahertz spin current pulses controlled by magnetic heterostructures. Nature nanotechnology, 8(4):256-60, April 2013.

[39] V. Zhukov, E. Chulkov, and P. Echenique. Lifetimes and inelastic mean free path of low-energy excited electrons in Fe, Ni, Pt, and Au: Ab initio GW+T calculations. Physical Review B, 73(12):125105, March 2006.

[40] Wei He, Tao Zhu, Xiang-Qun Zhang, Hai-Tao Yang, and Zhao-Hua Cheng. Ultrafast demagnetization enhancement in $\mathrm{CoFeB} / \mathrm{MgO} / \mathrm{CoFeB}$ magnetic tunneling junction driven by spin tunneling current. Scientific reports, 3:2883, January 2013.

[41] N. Wiese, T. Dimopoulos, M. Rührig, J. Wecker, G. Reiss, J. Sort, and J. Nogués. Strong temperature dependence of antiferromagnetic coupling in Co$\mathrm{FeB} / \mathrm{Ru} / \mathrm{CoFeB}$. Europhysics Letters (EPL), 78(6):67002, June 2007.

[42] W. Schweika. Schichtenpräparation mit Sputterverfahren. In Magnetische Schichtsysteme, 30. Ferienkurs des Instituts für Festkörperforschung, page A5. Forschungszentrum Jülich GmbH, 1998.

[43] P. Ehrhart. Präparation dünner Schichten: Molekularstrahl-Epitaxie. In Magnetische Schichtsysteme, 30. Ferienkurs des Instituts für Festkörperforschung, pages A4.1-A4.2. Forschungszentrum Jülich GmbH, 1998.

[44] Kai Ubben. Strukturierung von $\mathrm{CoFeB} / \mathrm{MgO} / \mathrm{CoFeB}-$ Tunnelmagnetowiderstands-Elementen, Diplomarbeit. 2009.

[45] Von Ardene. Von Ardenne Anlagentechnik GmbH, Betriebsanleitung Inverser Sputterätzer ISE 90. 2005.

[46] Johannes Christian Leutenantsmeyer. Master Thesis Parameter space for thermal spin-transfer torque. 2013. 
[47] Markus Münzenberg. Diplomarbeit, Untersuchungen zur Zwischenschichtenkopplung bei $[\mathrm{La} / \mathrm{Fe}]-$ Vielfachschichten und am Reorientierungsübergang von [CeH2/Fe]-Vielfachschichten mit dem magnetooptischen Kerreffekt. 1997.

[48] H. Sato, M. Yamanouchi, S. Ikeda, S. Fukami, F. Matsukura, and H. Ohno. Perpendicular-anisotropy $\mathrm{CoFeB}-\mathrm{MgO}$ magnetic tunnel junctions with a $\mathrm{MgO} / \mathrm{CoFeB} / \mathrm{Ta} / \mathrm{CoFeB} / \mathrm{MgO}$ recording structure. Applied Physics Letters, 101(2):022414, 2012.

[49] Hiroyuki Yamamoto, Jun Hayakawa, Katsuya Miura, Kenchi Ito, Hideyuki Matsuoka, Shoji Ikeda, and Hideo Ohno. Dependence of Magnetic Anisotropy in $\mathrm{Co}(20) \mathrm{Fe}(60) \mathrm{B}(20)$ Free Layers on Capping Layers in MgO-Based Magnetic Tunnel Junctions with In-Plane Easy Axis. Applied Physics Express, 5(5):053002, April 2012.

[50] Johannes Christian Leutenantsmeyer, Vladyslav Zbarsky, Steffen Wittrock, Marvin Walter, Patrick Peretzki, Henning Schuhmann, Andy Thomas, Karsten Rott, Guenter Reiss, Tae Hee Kim, Michael Seibt, and Markus Muenzenberg. Spin-transfer torque switching at ultra low current densities. arXiv.org, May 2014.

[51] Henning Schuhmann. Hochauflösende mikroskopische und spektroskopische Untersuchungen zur strukturellen Ordnung an MgO-CoFeB-Grenzflächen. $\mathrm{PhD}$ thesis, Georg-August-Universität Göttingen, 2014.

[52] Henning Schuhmann, Michael Seibt, Volker Drewello, Andy Thomas, Vladyslav Zbarsky, Marvin Walter, and Markus Münzenberg. Controlling boron redistribution in $\mathrm{CoFeB} / \mathrm{MgO}$ magnetic tunnel junctions during annealing by variation of cap layer materials and $\mathrm{MgO}$ deposition methods. arXiv.org, 2014.

[53] Marvin Walter, Jakob Walowski, Vladyslav Zbarsky, Markus Münzenberg, Markus Schäfers, Daniel Ebke, Günter Reiss, Andy Thomas, Patrick Peretzki, Michael Seibt, Jagadeesh S Moodera, Michael Czerner, Michael Bachmann, and Christian Heiliger. Seebeck effect in magnetic tunnel junctions. Nature materials, 10(10):742-6, October 2011.

[54] J. C. Leutenantsmeyer, M. Walter, V. Zbarsky, M. Münzenberg, R. Gareev, K. Rott, A. Thomas, G. Reiss, P. Peretzki, H. Schumann, M. Seibt, M. Czerner, and C. Heiliger. Parameter Space For Thermal Spin-Transfer Torque. SPIN, 03(01):1350002, March 2013.

[55] Hideo Sato, Michihiko Yamanouchi, Shoji Ikeda, Shunsuke Fukami, Fumihiro Matsukura, and Hideo Ohno. MgO/CoFeB/Ta/CoFeB/MgO Recording Structure in Magnetic Tunnel Junctions With Perpendicular Easy Axis. IEEE Transactions on Magnetics, 49(7):4437-4440, July 2013. 
[56] Mischa Bonn, Daniel Denzler, Stephan Funk, Martin Wolf, S.-Svante Wellershoff, and Julius Hohlfeld. Ultrafast electron dynamics at metal surfaces: Competition between electron-phonon coupling and hot-electron transport. Physical Review B, 61(2):1101-1105, January 2000.

[57] Chih-Wei Cheng, Wuwei Feng, G. Chern, C. M. Lee, and Te-ho Wu. Effect of cap layer thickness on the perpendicular magnetic anisotropy in top MgO/CoFeB/Ta structures. Journal of Applied Physics, 110(3):033916, 2011. 


\section{Nomenclature}

AP-Zustand Antiparalleler Zustand: entspricht der entgegengesetzten magnetischen Orientierung der ferromagnetischen Schichten eines Tunnelelements.

CoFeB Cobalt-Iron-Boron alloy (deutsch Kobalt-Eisen-Bor-Legierung). Wenn nichts anderes erwähnt, dann handelt es sich um eine 25:55:20 Zusammensetzung.

EELS Elektronenenergieverlustspektroskopie.

HRTEM High Resolution Transmission Electron Microscopy

IPA In-Plane Anisotropy

$\mathrm{MgO} \quad$ Magnesiumoxid

MOKE Magneto Optical Kerr Effect

MTJ Magnetic Tunnel Junction, magnetisches Tunnelelement

P-Zustand Paralleler Zustand: entspricht der gleichen magnetischen Orientierung der ferromagnetischen Schichten eines Tunnelelements.

PMA Perpendicular Magnetic Anisotropy

SQUID Superconducting Quantum Interference Device, supraleitende Quanteninterferenzeinheit

STT Spin-Transfer Torque

TEM Transmission Electron Microscopy

TMR Tunnel Magneto Resistance

TRMOKE Time-Resolved Magneto Optical Kerr effect, Zeitaufgelöster magnetooptischer Kerr-Effekt 



\section{Danksagung}

An erster Stelle möchte ich mich bei meinem Betreuer Prof. Dr. Markus Münzenberg für seine Betreuung, sein Vertrauen und für die Möglichkeit in der AG Münzenberg forschen zu können bedanken. Ich möchte mich auch für viele Jahre Zusammenarbeit und Spaß an Forschung bei der gesamten AG bedanken. Über die Jahre waren stets die Türen für eine Diskussion offen.

Beim Prof. Dr. Jooß möchte ich mich für die Übernahme des Koreferendariats bedanken.

Bei der gesamten Arbeitsgruppe möchte ich mich für viele Diskussionen und die wunderbaren Jahre bedanken. Insbesondere möchte ich mich bei Euch für folgendes bedanken:

- Marvin von der Ehe und Jakob Walowski für über 5 Jahre Zusammenarbeit, Diskussion und Unterstützung.

- Maria Mansurova für viele Diskussionen auch über nicht wissenschaftliche Themen.

- Tim Eggebrecht für viele Diskussionen sowohl zu wissenschaftlichen als auch zu weniger wissenschaftlichen Themen. Außerdem möchte ich mich für das Korrekturlesen und für eine ausgezeichnete Stimmung im Büro während des Zusammenschreibens bedanken.

- Ulrike Martens für die Proben, die sie für meine Experimente in kürzester Zeit gemacht hat.

- Dr. Henning Ulrichs für viele Ratschläge und Diskussionsbereitschaft.

- Der gesamten AG Ulrichs für viele Diskussionen und Korrekturlesen.

Diese Arbeit wäre ohne technische Unterstützung von U. Filippich, C. Mahn und der gesamten Werkstatt nicht möglich.

Ich möchte mich außerdem bei dem gesamten I. Physikalischen Institut für Unterstützung, Diskussionsfreude, Hilfsbereitschaft und eine familiäre Atmosphäre über die Jahre bedanken! 